\title{
Branched Flow and Caustics in Two-Dimensional Random Potentials and Magnetic Fields
}

\author{
Dissertation \\ zur Erlangung des Doktorgrades \\ der Mathematisch-Naturwissenschaftlichen Fakultäten \\ der Georg-August-Universität zu Göttingen
}

vorgelegt von

Jakob Johannes Metzger

aus Bonn

Göttingen 2010 
D7

Referent:

Prof. Dr. T. Geisel

Korreferent:

Prof. Dr. M. Timme

Tag der mündlichen Prüfung: 16.04.2010 


\section{Abstract}

Branched flow is a universal phenomenon of two-dimensional wave or particle flows which propagate through a weak random potential. Its origin is the formation of caustics, which are locations where the flow is focused by the cumulative effect of weak random forces acting along the flowpath. Branched flow has been observed on length scales spanning at least twelve orders of magnitude and in a variety of systems. For example, it has been studied in semiconductor microdevices, has been argued to be the mechanism underlying the formation of giant freak sea waves and has been predicted for the propagation of sound through the ocean on scales of several thousands of kilometers. A thorough understanding of the mechanism dictating how a random potential can cause such drastic effects such as branching is therefore important in many areas of physics, and is interesting to experimentalists and theoreticians alike.

In this thesis, we contribute to the theory of branched flow in the following ways. First, we consider the statistics of caustics along particle trajectories in a random potential with an additional deterministic focusing mechanism, a constant magnetic field. By extending existing theories and with detailed numerical simulations we can study the interplay between random focusing by the disorder potential and deterministic focusing by the magnetic field.

We then apply our theory to data from a magnetic focusing experiment in a semiconductor microstructure. We can reproduce the results of the experiments numerically and show them to be a result of random and deterministic focusing. Our results have important consequences for the conductance properties of semiconductor microstructures.

In the second part of the thesis, we consider the statistics of branches transverse to the flow, since this, although not as directly analytically and numerically accessible, is a quantity which can be measured more easily in an experiment. For the first time, we obtain statistics of the number of branches valid for all distances from a source, analytically and numerically. Also for the first time, we analyze the effect of different correlation functions and find an analytic expression for the universal curve describing the number of branches, which is valid for a wide range of correlation functions and parameters of the random potential. 


\section{Kurzfassung}

Branched flow ("verästelter Fluss") ist ein universelles Phänomen zweidimensionaler Teilchen- und Wellenflüsse, die durch ein schwaches, korreliertes Zufallspotential propagieren. Ihm liegt die Entstehung von Kaustiken zugrunde, an denen die Flussdichte besonders hoch ist. Die Flussverästelung ist auf Längenskalen, die sich auf mehr als zwölf Größenordnungen erstrecken, und in einer Vielzahl verschiedener Systeme beobachtet worden. Sie wurde unter anderem in Halbleiter-Mikrostrukturen untersucht, als Ursache für Riesenwellen (Monsterwellen) beschrieben und für die Propagation von Schallwellen durch den Ozean auf Skalen von tausenden von Kilometern vorhergesagt. Eine genaues Verständnis des Mechanismus, durch den ein schwaches Zufallspotential solch drastische Wirkung haben kann, ist daher in vielen Bereichen der Physik und für Experimentatoren ebenso wie für Theoretiker von großer Bedeutung.

In der vorliegenden Arbeit wird auf folgende Art und Weise zum theoretischen Verständnis der Flussverästelung beigetragen: Zuerst wird die Statistik von Kaustiken in einem Zufallspotential mit einem zusätzlichen deterministischen Fokussierungsmechanismus, einem konstanten Magnetfeld, betrachtet. Durch das Erweitern existierender Theorien und anhand detaillierter numerischer Simulationen kann das Zusammenspiel von zufälliger Fokussierung durch das Unordnungspotential und deterministischer Fokussierung durch das Magnetfeld untersucht werden.

Im folgenden werden die gewonnenen theoretischen Erkenntnisse zur Erklärung von Daten eines Experiments zur magnetischen Fokussierung in Halbleiter-Mikrostrukturen angewandt. Die experimentellen Daten können durch numerische Simulationen reproduziert werden. Es wird gezeigt, dass die Daten die Auswirkungen von deterministischer und zufälliger Fokussierung zeigen. Dieses Ergebnis hat wichtige Konsequenzen für die Transporteigenschaften von Halbleiter-Mikrostrukturen.

Im zweiten Teil der Arbeit wird die Statistik von Flussästen senkrecht zur Flussrichtung behandelt, da diese Größe, wenngleich numerisch und analytisch schwerer zugänglich, experimentell leichter zu messen ist. Erstmals wird die Statistik von Ästen, gültig für aller Entfernungen zur Quelle, analytisch und numerisch bestimmt. Ebenfalls zum ersten Mal wird der Einfluss unterschiedlicher Korrelationsfunktionen untersucht. Ein analytischer, universeller Ausdruck wird hergeleitet, der die Anzahl der Äste in Zufallspotentialen mit einer Vielzahl unterschiedlicher Korrelationsfunktionen und Parametern beschreibt. 


\section{Contents}

\begin{tabular}{lll}
\hline 1 & Introduction & 7
\end{tabular}

2 Fundamentals of caustics and branched flow 11

2.1 Lagrangian manifolds and caustics . . . . . . . . . . . . . . . . . . 12

2.2 Model of the random potential . . . . . . . . . . . . . . . . . . . 16

2.3 Branched flow and random caustics . . . . . . . . . . . . . . . 17

2.4 Catastrophes and branched flow . . . . . . . . . . . . . . . . . 18

2.5 Monodromy and its connection to caustics and intensities . . . . . . . . 19

2.6 Toy model of branch formation $\ldots \ldots \ldots \ldots$. . . . . . . . . . . 20

2.7 Conclusion . . . . . . . . . . . . . . . . . . . . . . . . . 24

3 Lagrangian caustic statistics in magnetic fields 25

3.1 Derivation of the curvature equation . . . . . . . . . . . . . . . 26

3.2 Derivation of the Fokker-Planck equation . . . . . . . . . . . . . . . 27

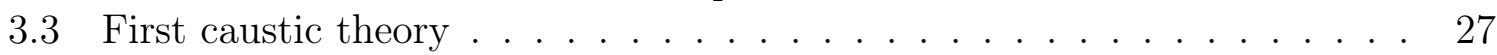

3.4 Magnetic focusing . . . . . . . . . . . . . . . . . . . . . . 30

3.5 First caustics in a magnetic field . . . . . . . . . . . . . . . . . . . 31

$3.6 \quad$ 2D equations and numerics for the curvature equation . . . . . . . . . . 34

3.7 Curvature equation with magnetic field . . . . . . . . . . . . . . . . . 38

3.8 Full first caustic distribution . . . . . . . . . . . . . . . . . . . . . . . . . 40

3.9 Conclusion $\ldots \ldots \ldots \ldots \ldots$. . . . . . . . . . . . . . . . . . . . . 41

4 Application: Magnetic Focusing $\quad 43$

4.1 Experimental setup and results . . . . . . . . . . . . . . . . . . . . . 44

4.2 Device geometry and its implications . . . . . . . . . . . . . . . . . 46

4.3 Simulation of the system with quantum point contacts and soft walls . . 49

4.4 Quantum correction . . . . . . . . . . . . . . . 50

4.5 Adding a random potential . . . . . . . . . . . . . . . . . . . . 51

4.6 Comparison of experimental and simulated data . . . . . . . . . . . . 53

4.7 Conclusion . . . . . . . . . . . . . . . . . . . . . . . 55

5 Measures of stability and their relation to fold and cusp caustics 57

5.1 Time evolution and initial conditions of the stability matrix . . . . . . 58

5.2 Cusps and the extended stability tensor . . . . . . . . . . . . . . 60

$5.3 \quad$ Analytic results and the quasi-2D approximation . . . . . . . . . . . . . 63 
5.4 Connection to curvature equation $\ldots \ldots \ldots \ldots$

5.5 Conclusion . . . . . . . . . . . . . . . . . . . . . . . . . 70

$\begin{array}{lll}6 & \text { Universal Branch Statistics } & 71\end{array}$

6.1 Counting caustics, counting branches . . . . . . . . . . . . . . . 72

6.2 Random initial condition without random potential . . . . . . . . . . . . 72

6.3 Counting branches numerically $\ldots \ldots \ldots \ldots$. . . . . . . . . . 73

6.4 Universal branch distribution . . . . . . . . . . . . . . . . . . . . . . . 75

6.5 Conclusion . . . . . . . . . . . . . . . . . . . 79

$\begin{array}{llr}7 \text { Conclusion and Outlook } & 81\end{array}$

\begin{tabular}{|ll}
\hline A Building a catastrophe machine & 85
\end{tabular}

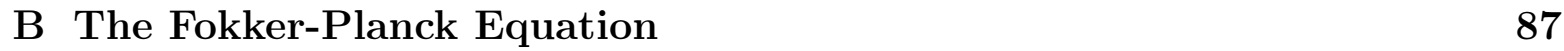

\begin{tabular}{ll}
\hline C Distribution incident on QPC & 90
\end{tabular}

\begin{tabular}{|lll}
\hline M Model potential at walls and QPCs of corner device & 91
\end{tabular}

\begin{tabular}{ll}
\hline Bibliography & 93
\end{tabular}

\begin{tabular}{ll}
\hline Acknowledgements & 101
\end{tabular} 


\section{Introduction}

Caustics appear abundantly in nature, and can be observed on almost all scales, from the microscopic to the macroscopic. Caustics are regions where a particle or wave flow is focused. They are therefore often the cause of extreme events and hence of special interest. Focused light, for example, is used in countless optical devices and supposedly was already employed by Archimedes, whose mirror construction was reported to have been powerful enough to ignite hostile ships [1]. In the ocean, underwater islands or other large-scale effects can focus water waves on the ocean surface and create huge freak waves [2-9]. Focused sound waves created by a plane crossing the sound barrier result in the sonic boom [10], a mechanism which is also used in particle physics, where Cherenkov light emitted from very fast particles is used to measure the particles' velocity $[11,12]$.

The theory of focusing has also been important in understanding natural phenomena, one of the most famous examples being Descartes' theory of the rainbow [13], and has sparked mathematical developments such as the Airy functions [14], which were first used to describe the intensity profile across a focal line.

The mathematical description of caustics has received considerable attention for at least two reasons. First, a focus is rarely just a point focus, which is in fact unstable and non-generic. Rather, caustics form intricate shapes and appear as lines and surfaces with complex topology. Secondly, they are, in the most elementary treatments (such as classical and standard semi-classical theories), singularities of a wave or particle flow, i.e. the flow density diverges at caustics, which complicates their mathematical treatment. Only in the 1970s and 80s, with the application of catastrophe theory to caustics, was it possible to classify the singularities rigorously and thereby to describe the different shapes which caustics take [15-20]. However, only few quantitative results have emerged from this approach $[21,22]$.

Focusing can not only take place in a specifically designed setup, but can also happen randomly. When particles or waves travel through a medium with small, random but correlated inhomogeneities, they can collectively focus the flow. In this way, even minute perturbations can quickly lead to drastic effects - a clear hint that the equations describing this phenomenon are nonlinear. There are many examples of this random focusing: The twinkling of starlight which propagates through the slightly inhomogeneous atmosphere [23-27] or light in an optical fibre focused by small perturbations [28], freak waves in the ocean which can even appear in calm seas and without an additional focusing mechanism such as an underwater island but just by small fluctuations in the wave velocity [29-37], the focusing of sound waves in the ocean because of the fluc- 


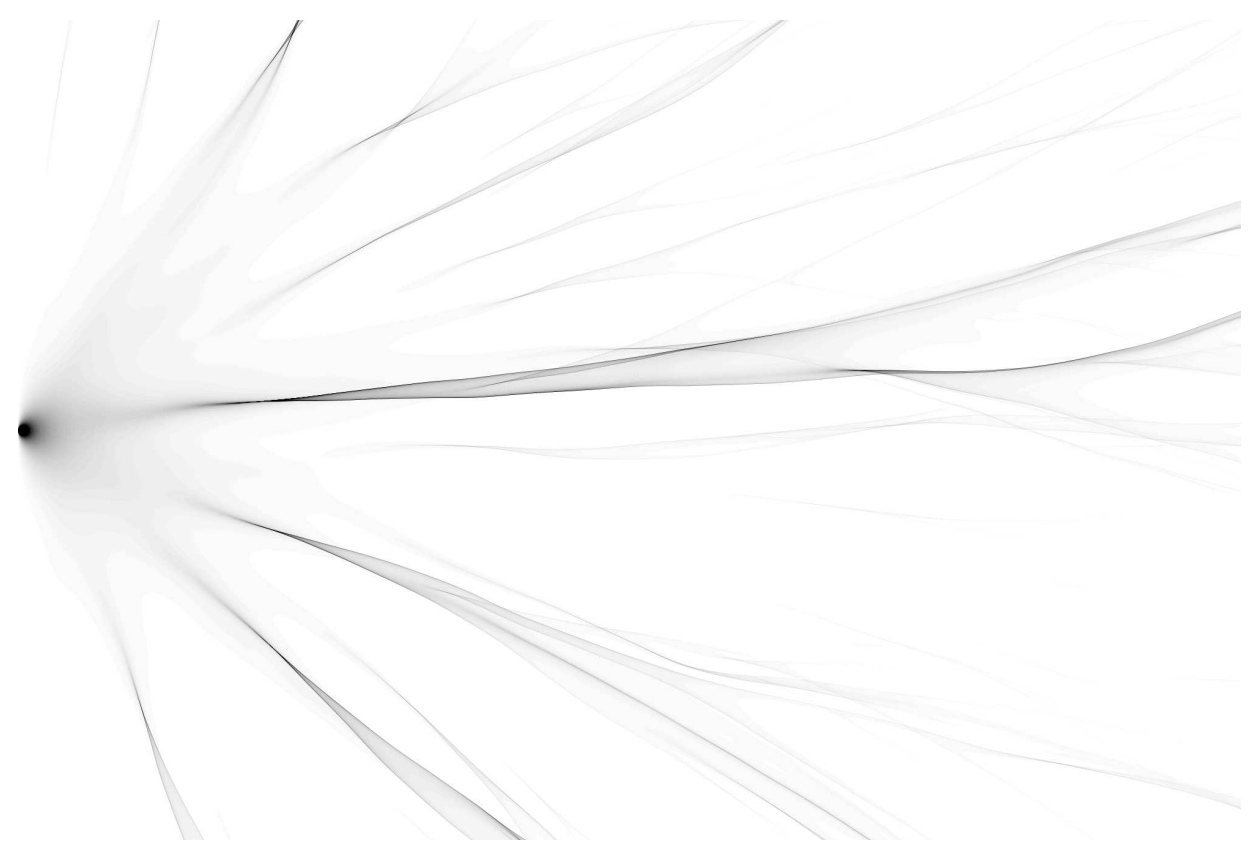

Figure 1.1: Flow from a point source through a random, correlated potential. Regions of high flow density are colored in dark gray.

tuating water density [38-44], and the random focusing of electrons in semiconductor devices because of a random disorder potential created by donor atoms [45-47], which can drastically alter the transport properties of such devices. Random caustics have also been described as a mechanism for the activation of rainshowers [48], and have recently been observed in microwave cavities [49]. A numerical simulation of a flow from a point source travelling through a random, correlated potential is shown in fig. 1.1. Branches of high intensity are clearly visible.

Although the fundamental and technological importance of understanding caustics in systems with random fluctuations is apparent, many questions in this field remain open. In this thesis, we, on the one hand, attempt to answer some of the fundamental, yet elusive questions in the theory of caustics in random media, and on the other hand apply the theory to the analysis of experimental data, showing the direct relevance of our theory. Since focusing is fundamentally a classical effect which is only softened and blurred in a corresponding wave system, we will deal almost exclusively with classical, conservative Hamiltonian systems. In addition, only two-dimensional systems are considered, as they allow an analytical treatment where, in many cases, the corresponding higher dimensional equations would not. Also, because of the complexity of the geometry of caustics in higher dimensions, it is much more complicated to observe them experimentally in more than two dimensions, which is reflected in the fact that many important examples of the appearance of caustics, such as most of the ones mentioned above, occur in systems which to a good degree of accuracy can be approximated as two-dimensional. 
The caustics then usually appear as pairs of lines resembling branches, which gives rise to the term branched flow. The thesis is set out in the following way:

In the first chapter, we describe fundamental concepts and tools needed for the analysis of caustics in random media. Here, we also further clarify the meaning of the term branched flow. However, it is not just a review of existing results, but already applies the concepts introduced to a toy model in order to provide some intuition and prepare the grounds for the following chapters.

In chapter two, we study the statistics of caustics along trajectories. We extend existing theories to allow for the calculation of caustic statistics of charged particles in a random potential, where in addition a constant magnetic field is applied. Since the magnetic field is by itself a focusing mechanism, we can study the interplay between this deterministic focusing and the random focusing by the disorder potential. We also perform numerical simulations for a wide range of random potentials to test existing results and to confirm our analytical results.

In chapter three, data from a magnetic focusing experiment in a semiconductor microdevice is analyzed. The theory developed in the previous chapter can be applied in order to understand where branching can take place and how it affects the experimental results. Together with detailed simulations of the experiment, we can explain the data as a results of magnetic and random focusing. Our results have important consequences for the conductance properties of semiconductor microdevices.

Chapter four shifts to a more technical delineation of the stability of the particle trajectories and how this can be used as an alternative approach to obtain caustic statistics to the one described in the second chapter. Not only simple caustics are treated, but a general method for finding caustics of higher order is devised using the example of the cusp. Stochastic equations for the quantities involved are derived, solved and compared to numerical simulations.

The final chapter describes the transition of caustic statistics along a trajectory to statistics in a fixed reference frame, i.e. the transition from a Lagrangian to an Eulerian view. Here, results from other chapters are combined in order to derive an approximate analytical expression for the number of branches per unit distance transverse to the flow and a certain distance away from the source, which is in excellent agreement with numerical simulations. We show that there exists a universal curve describing this quantity, valid for a wide range of random potentials. 



\section{Fundamentals of caustics and branched flow}

In this chapter, fundamental concepts and ideas which will be used throughout this thesis will be introduced. We begin by describing the theoretical framework which we will be working in and state some basic facts about the Hamilton-Jacobi-equation which are important for the theory of caustics and branched flow. We then study caustics in terms of Lagrangian manifolds, which provide an intuitive way of understanding the phase space geometry at caustics. Following this, we turn to a description of the density or intensity of the Hamiltonian flow in order to show that at caustics, the intensity of the flow diverges classically. This thesis is about transport through random media. Therefore, we also include a brief description of how the random potentials used in this thesis are modeled. This leads us to the meaning of the term branched flow in relation to caustics and random potentials, from where we move on to considering the structural stability and the geometry of caustics which will be done in the context of catastrophe theory. Following this, we introduce the stability matrix, which will be important throughout this thesis, and which provides a different approach to studying caustics. Finally, we construct the simplest possible model of cusp formation (without a random potential) in order to illustrate the quantities and concepts introduced in this chapter.

From a mathematical point of view, caustics appear ubiquitously in nonlinear partial differential equations (PDEs). In this thesis, they will be studied in the context of Hamiltonian flows, at the heart of which is the Hamilton-Jacobi-Equation (HJE) [16, $50-52]$,

$$
\frac{\partial}{\partial t} S(t, \vec{x})+H\left(t, \vec{x}, \frac{\partial S}{\partial \vec{x}}\right)=0
$$

where in the usual notation $S$ is the classical action, $H$ the Hamiltonian, $\vec{x}$ the position vector and $t$ the time. We will deal exclusively with Hamiltonians of the form

$$
H\left(t, \vec{x}, \frac{\partial S}{\partial \vec{x}}\right)=\frac{1}{2}\left(\frac{\partial S}{\partial \vec{x}}\right)^{2}+V(\vec{x})
$$

and thus will be considering conservative systems with non-interacting particles with mass equal to unity. The potential $V(\vec{x})$ will be a smooth, random function of the position. Since the HJE is a non-linear, first-order equation, it allows an analysis using the 
method of characteristics [53-55]. The characteristics of the HJE are the familiar trajectories which obey Newton's law. In any non-trivial Hamiltonian system, trajectories cross in finite time in position space, leading to a multivalued solution of the HJE. If the transition to a multivalued solution occurs smoothly, a caustic or focus forms. We begin the chapter by building a geometrical intuition using Lagrangian manifolds.

\subsection{Lagrangian manifolds and caustics}

\subsubsection{Heuristic definition}

Consider an action function $S(\vec{x})$ and its associated momentum vector field

$$
\vec{p}(\vec{x})=\frac{\partial S(\vec{x})}{\partial \vec{x}}
$$

with initial condition $\vec{p}_{0}\left(\vec{x}_{0}\right)$, and a swarm or bundle of particles which are described by their position and momenta. Then in phase space, because eq. (2.3) represents $n$ independent constraints on the $2 n$ variables $(\vec{x}, \vec{p})$, the bundle of particles is forced onto an $n$-dimensional surface. This surface is the Lagrangian manifold (LM), $\mathcal{L}$. It is the graph of the function $\vec{p}(\vec{x})$, i.e. the set of points in phase space of the form $(\vec{x}, \vec{p}(\vec{x}))[56]$. The surface thus created satisfies some differential constraints because of its derivation from a scalar in eq. 2.3. For example, it is immediately obvious that the momentum field $\vec{p}(\vec{x})$ is curl-free, i.e.

$$
\frac{\partial}{\partial x_{j}} p_{i}(x)=\frac{\partial}{\partial x_{j}} \frac{\partial S}{\partial x_{i}}=\frac{\partial}{\partial x_{i}} \frac{\partial S}{\partial x_{j}}=\frac{\partial}{\partial x_{i}} p_{j}(x) \quad i, j=1, \ldots, n .
$$

For a more precise definition, which also holds when the derivative of the action does not exist, see [56].

\subsubsection{Caustics, Lagrangian manifolds, curvature of the action function}

Since the LM is $n$-dimensional, one can impose $n$ new coordinates on it, $\left(h_{1}, \ldots, h_{n}\right)$. They label each point on $\mathcal{L}$ uniquely, and $\vec{x}$ and $\vec{p}$ on $\mathcal{L}$ can be given as $\vec{x}(\vec{h})$ and $\vec{p}(\vec{h})$. As long as $\vec{x}(\vec{h})$ is invertible, we can always write $\vec{p}(\vec{x})=\vec{p}(\vec{h}(\vec{x}))$. If, however, one approaches a point on $\mathcal{L}$ at which the determinant of the Jacobian $\operatorname{det}\left(d x_{i} / d h_{j}\right)$ vanishes, this is no longer possible, and the matrix

$$
\frac{\partial p_{i}}{\partial x_{j}}=\frac{\partial p_{i}}{\partial h_{k}} \frac{\partial h_{k}}{\partial x_{j}}
$$

will "behave badly" (in 1-d it will diverge because $\partial p / \partial h$ cannot be zero at the same time, in higher dimensions the exact behaviour also depends on $\left.\partial p_{i} / \partial h_{k}\right)[56,57]$. The 
set of points where $\partial x_{j} / \partial h_{k}$ vanishes is called the singular set, and the projection of this set onto configuration space are the places where caustics occur.

The formation of a caustic is illustrated for simplicity in a quasi-2D model. Here, the force in the first spatial coordinate, $x$, is ignored, and therefore is proportional to time (we choose it to be equal to $t$ w.l.o.g., we also call the second spatial coordinate $y$ ). In terms of the Lagrangian manifold, this means that a cut through the two-dimensional manifold at constant $x$ corresponds to the Lagrangian manifold of the quasi-2D system at time $t=x$. For illustration purposes, we choose a plane wave initial condition, which is defined by $x_{s}(0)=0, y_{s}(0)=s, p_{x, s}(0)=p_{0}, p_{y, s}(0)=0$, where $s$ is a parameter along the LM, and $p_{x}$ and $p_{y}$ denote the horizontal and vertical momentum, respectively. As $p_{x, s}(t)=p_{0}=$ const. and $x_{s}(t)=p_{0} t$ is independent of $s$, the manifold representing the bundle can be characterized at any time $t$ by a line in the $y-p_{y}$ or $y-v_{y}$ plane $\left(v_{y}\right.$ is the velocity in the $y$-direction). In fig. 2.1 an initially plane wave travels through a potential (green and white, the quasi-2D approximation means that the force in the $x$ direction is ignored) and develops a pair of caustics between $t_{4}$ and $t_{5}$. Here, the density (gray) is high, while in phase space (lower panel) the caustics can be identified as turning points of the Lagrangian manifold, marked in purple. At the turning point, two rays coalesce, and from here on the momentum and the action are multivalued.

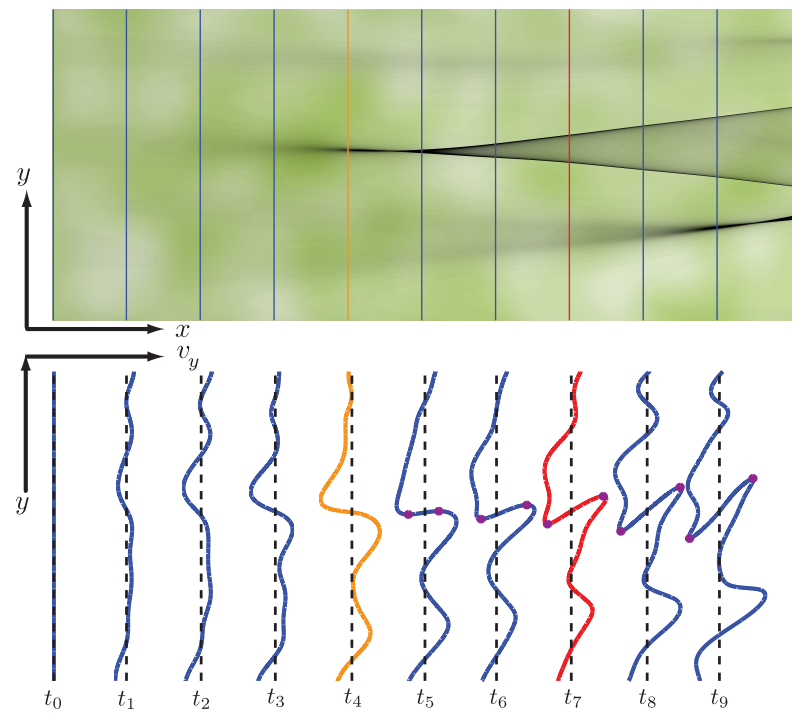

Figure 2.1: Plane wave flow (grey) and projections of LM onto configuration space (colored). Below the LM in phase space, with purple dots indicating turning points (caustics).

If one relaxes the quasi-2D condition, the turning points can also be observed in configuration space, as illustrated in fig. 2.2 with a point source initial condition in a full 2D potential. 


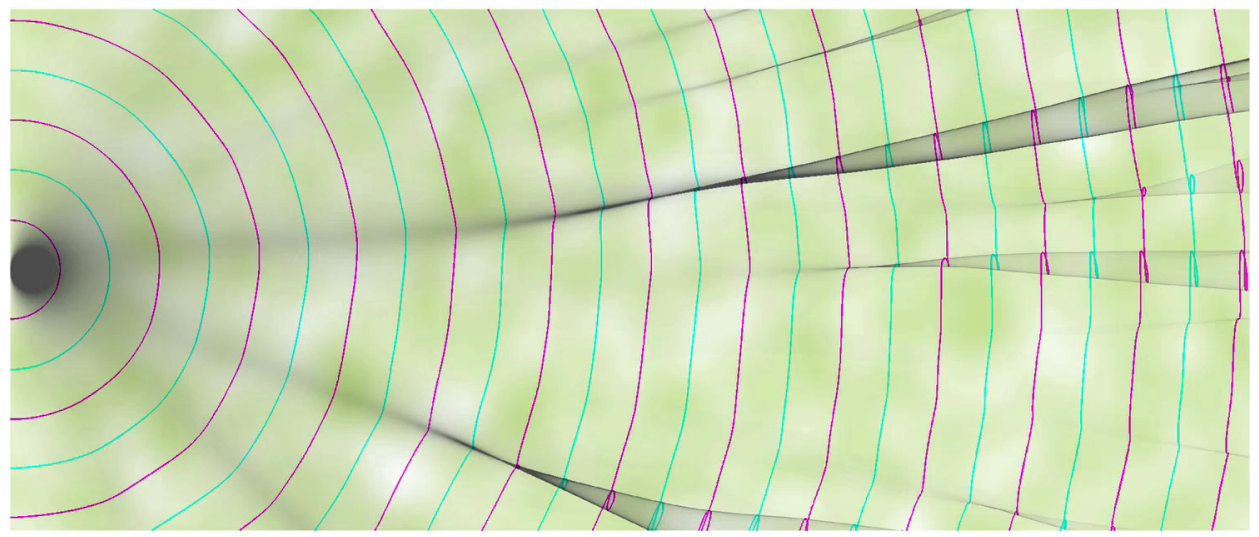

Figure 2.2: Point source with LM projections (colored). Turning points indicate caustic locations.

The location of the caustics can also be given as a condition on the curvature of the action $S$. The elements of the curvature matrix are given by

$$
u_{i j}=\frac{\partial^{2} S}{\partial x_{i} \partial x_{j}}=\frac{\partial p_{i}}{\partial x_{j}}
$$

which is, of course, eq. (2.5). The eigenvalues of this matrix, $\lambda_{i}$, are the principle curvatures of the action $[58,59]$. When one of the eigenvalues becomes infinite, a caustic is reached. This idea will be the basis of chapter 3, where equations for the curvature $u_{i j}$ and its eigenvalues $\lambda_{i}$ will be derived.

\subsubsection{Intensity along trajectories}

We now analyze the density that the rays carry and show that it becomes infinite at caustics. Consider again a ray bundle which is defined by the initial conditions $x_{s}(0)=$ $0, y_{s}(0)=s, p_{x, s}(0)=p_{0}, p_{y, s}(0)=0$ (plane wave initial condition). We derive the density generated by the ray bundle at a point $\vec{r}_{s}(t)=\left(x_{s}(t), y_{s}(t)\right)$ along the path of the trajectory with parameter $s$.

We start by taking a small initial area $\left(\vec{p}_{0} \Delta t\right) \times \vec{\delta}_{0}$ where $\vec{\delta}_{0}$ is along the initial Lagrangian manifold in position space, and $\delta_{0}$ its length. This area carries a weight of $\rho_{0}\left(\vec{p}_{0} \Delta t\right) \times \vec{\delta}_{0}=\rho_{0} p_{0} \Delta t \delta_{0}$, where $\rho_{0}$ is the initial (uniform) density. The density at $(t, \vec{x})$ is therefore given by

$$
\rho(t, \vec{x})=\sum_{\text {traj }} \frac{\rho_{0} p_{0} \Delta t \delta_{0}}{|(\vec{p}(t) \Delta t) \times \vec{\delta}(t, \vec{x})|}=\sum_{t r a j} \frac{\rho_{0} p_{0}}{\left|\vec{p}(t) \times \frac{\partial \vec{x}}{\partial s^{\prime}}\right| \frac{\partial s^{\prime}}{\partial s}}
$$

where the sum is over all trajectories which cross the point $(t, \vec{x})$ and $s^{\prime}$ now parametrizes the manifold at time $t$. For the quasi-2D case, where $x=t, p_{0}=1$ and the spatial 


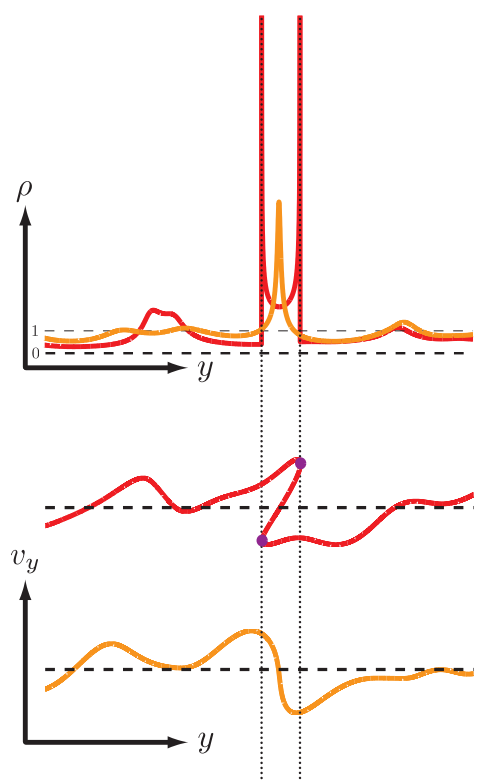

Figure 2.3: Densities $\rho$ for the flow in fig. 2.1. Lower two panels are the corresponding LMs. At the turning points, the density becomes classically infinite. At the center of the branch, there is an approximately three-fold increase of the density because the region is covered three times with the LM (there are always three trajectories at every point).

variable is $y$, this becomes

$$
\rho_{\text {quasi-2D }}=\sum_{\text {traj }} \frac{\rho_{0}}{\frac{\partial y}{\partial s^{\prime}} \frac{\partial s^{\prime}}{\partial s}}
$$

from which two different contributions to the density can be observed. The first is the projection of the manifold onto real space, $\partial y / \partial s^{\prime}$, which, when it is zero, is responsible for the caustics. The second is the stretching of the manifold along the direction of its parametrization, $\partial s^{\prime} / \partial s$, which gives an additional multiplicative change in the density. We can also write this expression in terms of the curvature defined in eq. (2.6) as follows. Since

$$
\delta s^{\prime}=\sqrt{\delta y^{2}+\delta p_{y}^{2}}=\delta y \sqrt{1+\left(\partial p_{y} / \partial y\right)^{2}}=\delta y \sqrt{1+u^{2}}
$$

we can write eq. (2.8) as

$$
\rho_{\text {quasi-2D }}=\sum_{\text {traj }} \frac{\rho_{0}}{\frac{\partial y}{\partial s^{\prime}} \frac{\partial s^{\prime}}{\partial s}}=\sum_{t r a j} \rho_{0} \frac{\partial s}{\partial s^{\prime}} \sqrt{1+u^{2}} .
$$

In fig. 2.3, the densities $t_{4}$ and $t_{7}$ from fig. 2.1 are plotted with their respective LM.

\subsubsection{Intensities at caustics}

Since the shape of the manifold directly at the caustic can be calculated, the density close to it can be inferred from this approximately. We will use the quasi-2D model for 
simplicity.

At a fold caustic (see sec. 2.4 for exact definition), the line in $y-p_{y}$ space has one turning point, which means that the lowest term in the Taylor expansion is therefore $y=p_{y}^{2}$ or $p_{y}= \pm y^{1 / 2}$. At a cusp, two turning points coalesce, which means a relation of the form $y=p_{y}^{3}$. From eq. 2.9, we can then calculate that the density transverse to a fold scales like

$$
\rho_{\text {fold }} \propto \sqrt{1+\left(\frac{\partial p_{y}}{\partial y}\right)_{\text {fold }}^{2}}=\sqrt{1+\frac{1}{4 y}} \approx \frac{1}{2} y^{-1 / 2}
$$

and across a cusp like

$$
\rho \text { cusp } \propto \sqrt{1+\left(\frac{\partial p_{y}}{\partial y}\right)_{\text {cusp }}^{2}}=\sqrt{1+\frac{1}{9 y^{4 / 3}}} \approx \frac{1}{3} y^{-2 / 3}
$$

to first order.

\subsection{Model of the random potential}

In this thesis, we consider flow through random media or potentials. The model used for the random medium is a 2D stationary Gaussian random field with a correlation function which has one spatial length scale but is arbitrary otherwise, as long as it fulfills the usual conditions for a correlation function. It must be twice differentiable at the origin (otherwise leading to a fractal potential without a well-defined length scale) and its Fourier transform (FT) must be positive definite (since by the Wiener-Khinchin theorem the FT of the autocorrelation is the power-spectrum which is positive definite) [60,61]. We will see in the following chapters, that we will additionally require the correlation function to be up to six times differentiable at the origin, in order to obtain analytics results on the statistics of random caustics. The correlation function is then given by

$$
c(\vec{x})=\left\langle V\left(\vec{x}^{\prime}\right) V\left(\vec{x}^{\prime}+\vec{x}\right)\right\rangle=\epsilon^{2} f_{c}\left(\vec{x}, \ell_{c}\right)
$$

where $f_{c}$ fulfills the above-mentioned criteria but is otherwise an arbitrary function of the correlation length $\ell_{c}$, and $\epsilon$ is the standard deviation of the Gaussian point process at any $\vec{x}$, i.e. the strength of the random potential. The potential is constructed by convoluting the correlation function with a random phase field. After taking the Fourier transform of the correlation function, the square root of the Fourier components is taken, which are then multiplied by phases $2 \pi \phi(\mathbf{x})$ with $\phi(\mathbf{x})$ distributed uniformly between $[0,1)$, and then transformed back to real space:

$$
V(\vec{x})=\mathcal{F} \mathcal{T}^{-1}\left[\sqrt{\mathcal{F} \mathcal{T}[c(\vec{x})]} e^{2 \pi i \phi(\vec{x})}\right]
$$

where $\mathcal{F} \mathcal{T}$ denotes the Fourier transform. This yields a Gaussian random field $V(\vec{x})$ because of the central limit theorem (the Fourier transform here is a sum of independent 


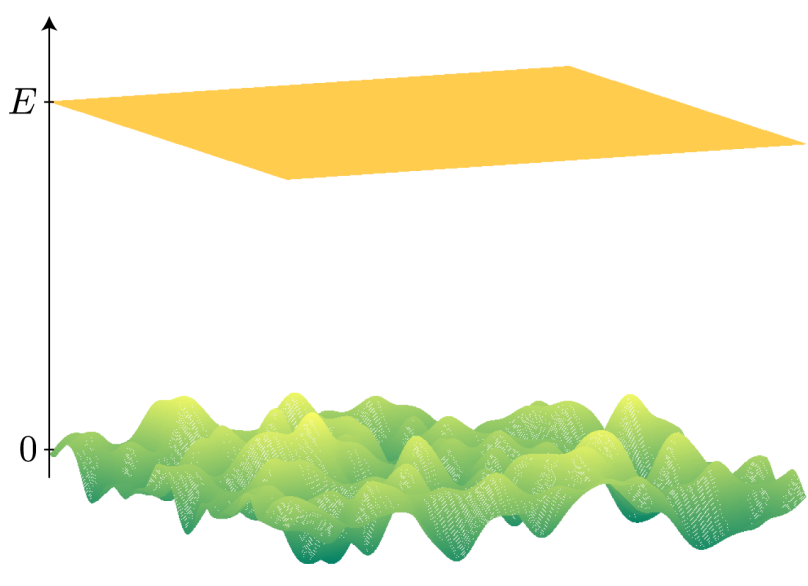

(a)

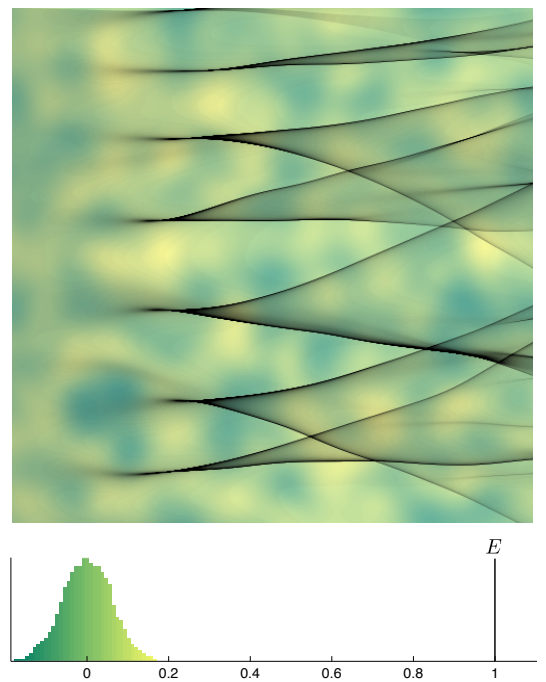

(b)

Figure 2.4: (a) A random potential with a Gaussian correlation function. The orange plane corresponds to the energy of the particles, E. (b) An initially plane flow develops caustics, caused by a random potential (colored). The potential used here is the same as in (a). Below we show a histogram of the values of the random potential used above with the same color code. The potential is clearly very weak compared to the energy of the flow (here normalized to one).

random variables because of $\phi(\vec{x}))$. A picture of a potential generated in this manner is shown in fig. 2.4a.

Numerically, the potential is calculated on a grid and points in between are interpolated using 2D splines (code developed in [62]). It should be emphasized that the random potentials used throughout this thesis are weak compared to the energy of the particles that constitute the flow. This is illustrated in fig. $2.4 \mathrm{~b}$, where a flow is shown together with a histogram of the random potentials underlying the flow. Also indicated is the energy of the particles, which we denote by $E$.

\subsection{Branched flow and random caustics}

The term branched flow is employed when caustics are caused by a weak, correlated, random potential and form lines, which, possibly washed out by quantum or other wave effects and observed from some distance, resemble branches of a tree. A branch is always bounded by two caustics and has an increased density at its core, compared to its surroundings, as shown in fig. 2.3. An example of this is the electron flow in a twodimensional electron gas, such as seen in [45], from which we take fig. 2.5. Since in this 


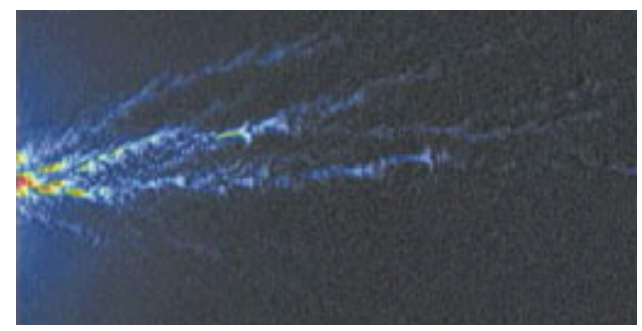

Figure 2.5: Scanning probe microscopy image of a branched flow in a two-dimensional electron gas (from [45]). Electrons flow out of a point contact on the left. Branches, resulting from a weak potential that deflects the flow, are clearly visible.

thesis we consider flow through weak, correlated disorder potentials, the caustics will always take the shape of branches.

\subsection{Catastrophes and branched flow}

We have seen that caustics are singularities of the ray density, and that they occur where the action $S(\vec{x})$ becomes multivalued. Catastrophe theory can explain the shapes the caustics take as follows:

The multivalued $S(\vec{x})$ is embedded into a function $\phi(\vec{s}, \vec{x})$ such that

$$
\partial \phi / \partial s_{i}=0
$$

is the condition for the trajectories, which is represented by a (possibly infinite) set of $s_{i}[16,20]$. For example, one can think of the trajectory being split up into many small pieces, with each piece being described by two of the $i$ variables $s_{i}$. In our case, we have a smooth random potential deflecting a particle at every instant. We would therefore require a continuous set of $s_{i}$.

In catastrophe theory, $\phi$ is called a generating function, since the trajectories can be derived from the gradient map eq. (2.12). When two trajectories coalesce, not only eq. (2.12) is fulfilled, but $\phi$ is also stationary to higher order, i.e. (2.12) becomes singular, and the caustics are equivalent to singularities of a gradient map. Catastrophe theory can now determine the stability of these singularities, which are the catastrophes. For this, each separate branch of the action is equipped with an index $\mu$, such that $S_{\mu}(\vec{x})$ is now unique, and

$$
S_{\mu}=\phi\left(s^{\mu}(\vec{x}), \vec{x}\right)
$$

are the actions of the branches. The $s_{i}$ are called state variables, the $\vec{x}$ and all other possible parameters are termed control parameters, denoted by $C=\left(C_{1}, C_{2}, \ldots\right)$. The catastrophes can now be classified according to their codimension $K$, which is the number of control parameters $C$ minus the dimensionality of the catastrophe, as is done below. 

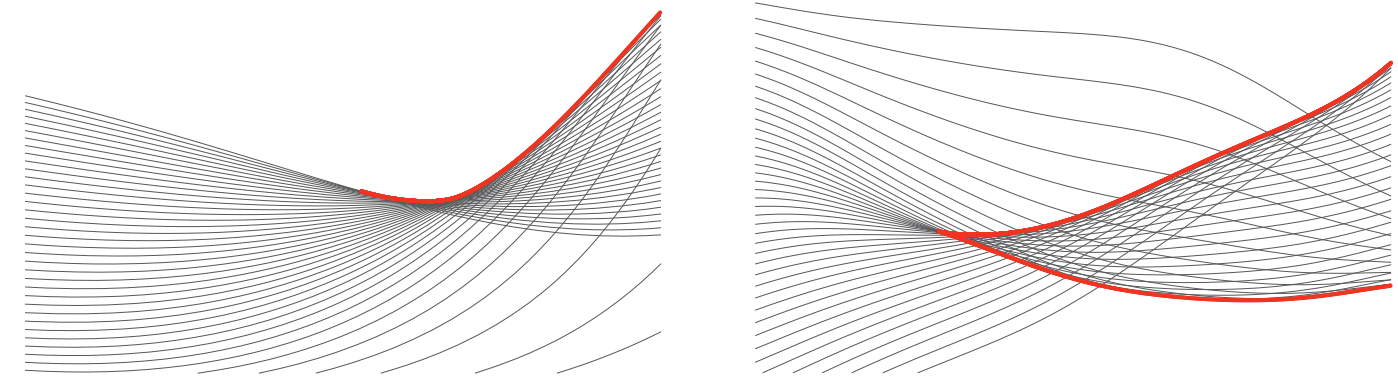

Figure 2.6: The possible stable catastrophes in a two-dimensional random potential, the fold and the cusp unfolding into two folds. The latter case is the one encountered generically in a random potential.

Most importantly, the catastrophes with codimension less than seven have been shown to be structurally stable, i.e. they retain their form under perturbations. More precisely, the perturbed action $S^{\prime}$ is related to the original $S$ by a diffeomorphism of $C$, and the generating function $\phi$ can be cast into standard form, a characteristic polynomial $[16,18,20,63]$.

In the 2D, stationary branched flows studied in this thesis there are two control parameters. These are the spatial coordinates of the observer, $x$ and $y$. The possible (stable) catastrophes which appear in this thesis are therefore the cusp (codimension $2-0=2$ ) and the fold (codimension $2-1=1$ ). The cusps are said to organize the folds, which means that every pair of folds has a cusp at their common origin. Fold and cusp are illustrated in fig. 2.6.

For illustratory and pedagogical purposes we have built a catastrophe machine, the details of which can be found in appendix A.

\subsection{Monodromy and its connection to caustics and intensities}

The monodromy or stability matrix $\mathbf{M}$ is an important concept in the study of dynamical systems. It describes how a small volume of phase space is changed over time and will be studied in more detail in chapter 5. For this introduction, we content ourselves with giving the two-dimensional form

$$
\left(\begin{array}{c}
\delta x(t) \\
\delta y(t) \\
\delta p_{x}(t) \\
\delta p_{y}(t)
\end{array}\right)=\mathbf{M}(t)\left(\begin{array}{c}
\delta x(0) \\
\delta y(0) \\
\delta p_{x}(0) \\
\delta p_{y}(0)
\end{array}\right), \quad \mathbf{M}(0)=1
$$


with obvious extension to other dimensions [42,64,65]. The time evolution of $\mathbf{M}(t)$ is given by

$$
\dot{\mathbf{M}}(t)=\left(\begin{array}{cc}
0 & \mathbb{1} \\
-\mathbb{1} & 0
\end{array}\right)\left(\frac{\partial^{2} H}{\partial \gamma_{i} \partial \gamma_{j}}\right)=\mathbf{K}(t) \mathbf{M}(t)
$$

where $\gamma_{i}$ is a phase space variable. The monodromy matrix can be used to classify the motion as stable or unstable and is linked to the Lyapunov exponent (see chap. 5).

The monodromy matrix can also be used to find caustics and to calculate flow densities by observing that the small piece of manifold $\vec{\delta}$ from $(2.7)$ is equal to the projection onto position space of the time-transported piece $\vec{\delta}_{0}$ of the initial manifold in phase space, i.e. $\vec{\delta}(t)=\mathbf{P}_{\text {pos. space }}\left(\mathbf{M}(t) \vec{\delta}_{0}\right)$, where $\mathbf{P}$ is the projection operator to position space. Then the flow density is

$$
\rho(t, \vec{x})=\sum_{\text {traj }} \frac{\rho_{0} p_{0} \Delta t \delta_{0}}{|(\vec{p} \Delta t) \times \delta(t, \vec{x})|}=\sum_{\text {traj }} \frac{\rho_{0} p_{0} \delta_{0}}{\left|\vec{p} \times \mathbf{P}_{\text {pos. space }}\left(\mathbf{M} \vec{\delta}_{0}\right)\right|}
$$

where the sum is over all trajectories at $(t, \vec{x})$. The condition for the density to diverge is then the condition for a caustic, i.e.

$$
\vec{p} \times \mathbf{P}_{\text {pos. space }}\left(\mathbf{M} \vec{\delta}_{0}\right)=\left(-p_{y}, p_{x}, 0,0\right)^{T} \mathbf{M} \vec{\delta}_{0}=0 .
$$

In chapter 5 this will be studied in more detail for several initial conditions, and the concept of the stability matrix itself will be extended.

\subsection{Toy model of branch formation}

As discussed above, the only stable caustic structure in a 2D random medium is the cusp with two fold lines. We construct a simple toy model which allows us to study all the concepts mentioned above analytically.

\subsubsection{Model}

We use the simplest model, a pseudo-2D cusp in a zero potential which is determined just by its initial conditions (in particular, the initial angle or velocity). This is similar in spirit to a model used in [66], although both the equations and the aims are different.

The initial condition for the density is a plane wave of constant density. A Liouville density approach is used to propagate the initial conditions across the cusp. Since inside a cusp, the action function will be (at least) three-valued, we choose the simplest initial condition which allows us to solve "only" a cubic equation (and not a quartic or higher order equation). The equation of motion and the initial conditions we choose are

$$
\begin{aligned}
y & =f_{x}\left(y_{0}\right)=y_{0}+p\left(y_{0}\right) x \\
p\left(y_{0}\right) & =-\frac{b y_{0}}{1+a^{2} y_{0}^{2}} .
\end{aligned}
$$




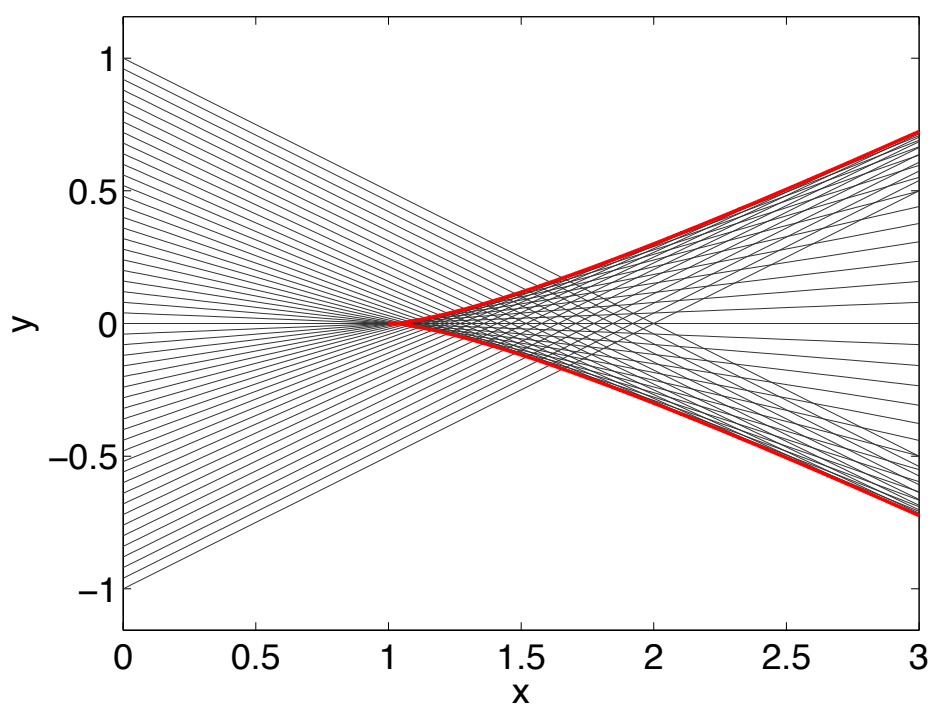

Figure 2.7: Simple model which produces a cusp from which two fold caustics emerge $(a=1)$. The caustics (red) are the envelopes of the trajectories (black lines), and appear where the discriminant $\Delta=0$.

We will need, for a given position $(x, y)$, to be able to determine the initial $y_{0}$ and therefore need to invert the equations above to get $y_{0}(x, y)$. Now the particular form of $p\left(y_{0}\right)$ becomes apparent, since attempting to solve for $y_{0}$ yields

$$
y_{0}^{3}-y y_{0}^{2}+\left(-b / a^{2} x+a^{-2}\right) y_{0}-a^{-2} y=0
$$

which is cubic in $y_{0}$ as desired. For our purposes, we can set $b=1$ and retain all important features.

From the discriminant $\Delta$ one can infer whether there is one or three distinct real roots to the equation. It is given by [53]

$\Delta=4 a^{-2} y^{4}-y^{2}\left(-x a^{-1}+a^{-2}\right)+4\left(-x a^{-1}+a^{-2}\right)^{3}-18 y^{2} a^{-2}\left(-x a^{-1}+a^{-2}\right)+27 a^{-4} y^{2}$.

For $\Delta<0$ there exist three real roots, whereas for $\Delta>0$ there is one real root. The border of this region, where the action function goes from being single valued to threevalued is just the cusp which is implicitly given by the equation $\Delta=0$ (fig. 2.7).

\subsubsection{General expression for the flow density}

In order to obtain an expression for the flow density we propagate the initial density $\rho_{0}=\rho(0, y)=$ const. to some later value using the Frobenius-Perron operator $[67,68]$

$$
\rho(x, y)=\int d y_{0} \delta\left(y-f_{x}\left(y_{0}\right)\right) \rho_{0}(y)=\sum_{y_{0}=f_{-x}(y)} \frac{\rho_{0}(y)}{\left|\frac{\partial y}{\partial y_{0}}\right|}
$$


where the Jacobian in the denominator is evaluated at $y_{0}$, which is why we need solutions to 2.13 . Setting the constant $\rho_{0}$ to unity, we can now give the density as

$$
\rho(x, y)=\left\{\begin{array}{ccc}
\left|J^{-1}\left(y_{1}\right)\right| & \text { if } & \Delta(x, y)>0 \\
\infty & \text { if } & \Delta(x, y)=0 \\
\sum_{i=1}^{3}\left|J^{-1}\left(y_{i}\right)\right| & \text { if } & \Delta(x, y)<0
\end{array}\right.
$$

where the Jacobian is given by

$$
J\left(x, y_{0}\right)=1-\frac{1-a^{2} y_{0}^{2}}{\left(1+a^{2} y_{0}^{2}\right)} a x
$$

and the three roots of $y_{0}$ (of which $y_{1}$ is always real) are

$$
\begin{aligned}
& y_{1}=-\beta / 3-\frac{2^{1 / 3}\left(-\beta^{2}+3 \gamma\right)}{\nu}+\frac{\nu}{32^{1 / 3}} \\
& y_{2}=-\beta / 3+(1+i \sqrt{3}) \frac{\left(-\beta^{2}+3 \gamma\right)}{32^{2 / 3} \nu}-(1-i \sqrt{3}) \frac{\nu}{6 \cdot 2^{1 / 3}} \\
& y_{3}=-\beta / 3+(1-i \sqrt{3}) \frac{\left(-\beta^{2}+3 \gamma\right)}{32^{2 / 3} \nu}-(1+i \sqrt{3}) \frac{\nu}{6 \cdot 2^{1 / 3}}
\end{aligned}
$$

with $\omega, \nu$ introduced to make the equations compact and $\beta, \gamma, \delta$ as the coefficients of the cubic equation (2.13):

$$
\begin{aligned}
\omega & =\sqrt{4 \beta^{3} \delta+27 \delta^{2}-18 \beta \delta \gamma-\beta^{2} \gamma^{2}+4 \gamma^{3}} \\
\nu & =\left(-2 \beta^{3}-27 \delta+9 \beta \gamma+3 \sqrt{3} \omega\right)^{1 / 3} \\
\beta & =y \\
\gamma & =-a^{-1} x+a^{-2} \\
\delta & =a^{-2} y .
\end{aligned}
$$

Because of symmetry, the cusp begins at $y=0$ and $\Delta(x, 0)=0$ from which it follows for the $x$-value of the cusp

$$
x_{\text {cusp }} \stackrel{(y=0)}{=} a^{-1} \text {. }
$$

\subsubsection{Density at cusp along $\mathrm{y}$}

We would now like to derive an expression for the density directly at the cusp point and compare its scaling behaviour with the one predicted in eq. (2.10). At the cusp, the expression for the Jacobian (2.15) becomes 


$$
J\left(a, y_{0}(y)\right)=1-\frac{1-a^{2} y_{1}^{2}}{\left(1+a^{2} y_{1}^{2}\right)}
$$

Taylor expanding at the cusp in the $y$ - direction gives

$$
\rho(x=1 / a, y)=1 / 6+\frac{1}{2} y^{-2 / 3}+\mathcal{O}\left(y^{2 / 3}\right)
$$

which scales as $y^{-2 / 3}$ to first order, just as predicted in sec. 2.1.4.

\subsubsection{Monodromy}

In the pseudo-2D case $x=t$, and for this simple model with $V=0$ the equations for the monodromy become

$$
\begin{aligned}
\mathbf{M}(0) & =\mathbb{1} \\
\dot{\mathbf{M}}(t) & =\mathbf{K} \mathbf{M}=\left(\begin{array}{cc}
0 & -\frac{\partial^{2} V}{\partial y^{2}} \\
1 & 0
\end{array}\right) \mathbf{M}=\left(\begin{array}{ll}
0 & 0 \\
1 & 0
\end{array}\right) \mathbf{M}=\left(\begin{array}{cc}
0 & 0 \\
m_{11}(t) & m_{12}(t)
\end{array}\right) \\
\Rightarrow \mathbf{M}(t) & =\left(\begin{array}{ll}
1 & 0 \\
t & 1
\end{array}\right) .
\end{aligned}
$$

This can be used to propagate the initial manifold ( $\delta p$ denotes the momentum in $y$ direction, and the prime a derivative with respect to $\left.y_{0}\right)$ :

$$
\begin{aligned}
\left(\begin{array}{c}
\delta y \\
\delta p
\end{array}\right) & =\left(\begin{array}{c}
t \delta p_{0}+\delta y_{0} \\
\delta p_{0}
\end{array}\right)=\left(\begin{array}{c}
t p_{0}^{\prime}\left(y_{0}\right)+1 \\
p_{0}^{\prime}\left(y_{0}\right)
\end{array}\right) \delta y_{0} \\
p_{0}^{\prime}\left(y_{0}\right) & =\frac{-b\left(1+a^{2} y_{0}^{2}\right)+2 b a^{2} y_{0}^{2}}{\left(1+a^{2} y_{0}^{2}\right)^{2}}=\frac{-b\left(1-a^{2} y_{0}^{2}\right)}{\left(1+a^{2} y_{0}^{2}\right)^{2}}
\end{aligned}
$$

The density contributed by a single trajectory is, from eq. 2.14, simply the inverse of the matrix element $m_{11}, \rho(x, y)=\left(\delta y / \delta y_{0}\right)^{-1}$

$$
\delta y / \delta y_{0}=x p_{0}^{\prime}\left(y_{0}\right)+1=x p_{0}^{\prime}\left(y_{0}(y)\right)+1=\frac{-b x\left(1-a^{2} y_{0}^{2}\right)}{\left(1+a^{2} y_{0}^{2}\right)^{2}}+1 .
$$

This is consistent with 2.15) and (2.16), and completes our analytical treatment of the toy model.

A plot for the density is shown in fig. 2.8. Also displayed are several vertical cuts through the density shortly before the cusp begins (in red), at the cusp (lighter red) and equidistantly after the cusps. 

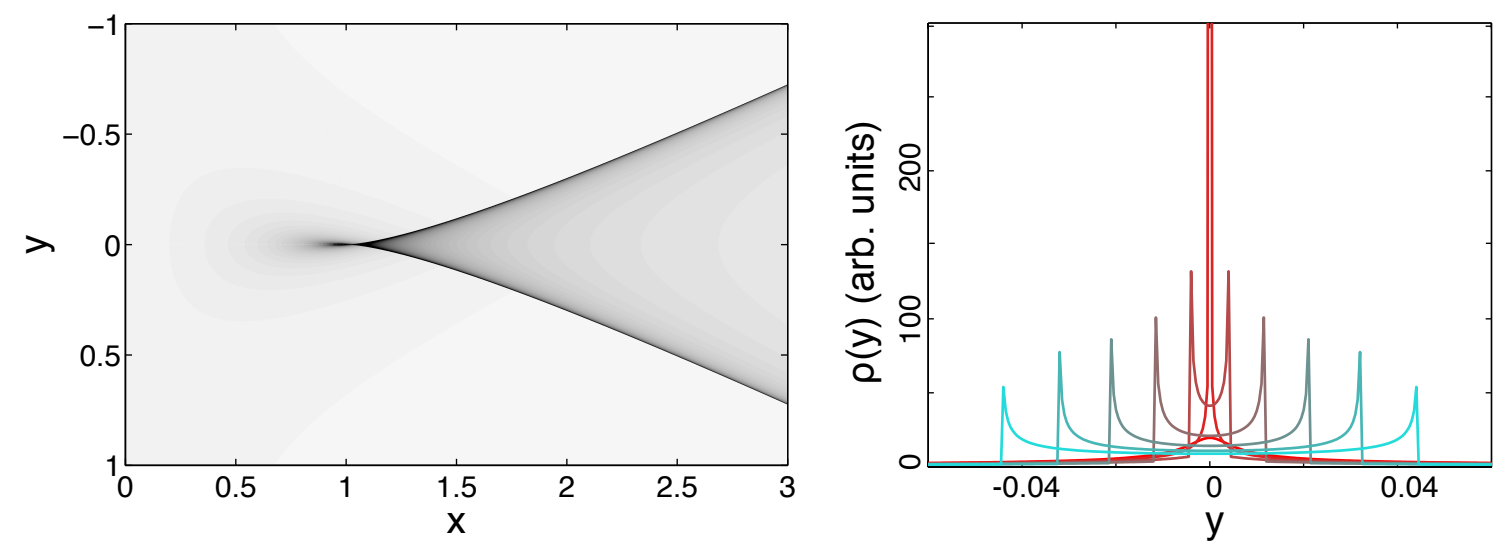

Figure 2.8: Density $\rho(x, y)$ and cuts through $\rho(x, y)$ at several constant values of $x$.

\subsection{Conclusion}

In this chapter, we have introduced the basic ingredients of this thesis. We have shown that caustics are singularities of the classical flow, and have analyzed their phase-space geometry using Lagrangian manifolds. This has also allowed us to calculate how the intensity scales across different types of caustics. These types can be classified using catastrophe theory, and we have shown that in a random potential the caustics generically appear as pairs of lines (or folds in the language of catastrophe theory), which emerge from a cusp. The appearance of these structures in a random potential is called branched flow. We have also shown how to calculate the location of caustics using the stability matrix and have applied all the concepts to a toy model of branch formation. 


\section{Lagrangian caustic statistics in magnetic fields}

In the previous chapter, we have seen that even a very weak random potential quickly focuses a flow from an ordered source, and that these random foci appear as branches of high intensity. We have interpreted this in terms of Lagrangian manifolds associated with the flow, which stretch and fold, thereby creating caustics at random locations. The locations of the caustics are related to the characteristics of the underlying disorder potential. Therefore, by observing caustics, one can infer from this information about the random potential, and when the characteristics of the random potential are known, one can predict the statistics of the caustics. In this chapter, we therefore study the statistics of the random caustics in terms of the parameters of the random potential.

First, we review some of the results on the statistics of caustics and test them numerically. We then ask a further question: How does a random potential influence a deterministic focusing device? This is important since in any experiment which employs a focusing device there are small perturbations, leading themselves to random focusing. We study this by adding a constant magnetic field to our equations. The magnetic field focuses charged particles and allows us to study the interplay between this deterministic focusing and the random focusing of the disorder potential. Our theory will be applied to a transverse magnetic focusing experiment in semiconductor microstructures in the next chapter.

We begin our detailed analysis of the statistics of caustics by using a Lagrangian approach which has been widely used in the literature. It consists of changing the equations derived from the Hamilton-Jacobi-Equation (HJE) to ordinary differential equations along the characteristics of the HJE, which are the trajectories. This method is very useful, since it will allow us to analyze an equation which will serve to identify the caustics along a trajectory within the framework of ordinary stochastic differential equations (OSDE). This means that we can use the well-established tools of OSDEs such as the Fokker-Planck equation. Since in the derivation of the Fokker-Planck equation, it is assumed that the stochasticity enters as white noise in the time domain, we need to make an approximation which will be used in all the analytical calculations in this thesis: In a weak random potential, the particles move in their main propagation direction very quickly. This direction is identified with time (quasi-2D approach) and since the particles move across the potential quickly in this direction, it is assumed that it appears as an almost uncorrelated series of random numbers. The assumption is therefore that the 
correlation function in time is just the delta function, and that the characteristics of the random potential appear in the prefactor of the delta function, which will depend on the parameters of the random potential.

With this in mind, we begin this chapter by deriving an equation for the curvature of the action from the HJE and its corresponding FPE, as was introduced in the previous chapter, sec. 2.1. From this, we perform a first passage time calculation to obtain statistics of the location of caustics. We then introduce a magnetic field as a different focusing mechanism. We also derive detailed initial conditions for the elements of the curvature matrix. As in the remainder of the thesis, we will focus on the two most important initial conditions, the point source and the plane wave. However, the method used to derive them can in principle be applied to different initial conditions as well.

\subsection{Derivation of the curvature equation}

Consider again the HJE (2.1) in the form

$$
\frac{\partial}{\partial t} S(t, \vec{x})+\frac{1}{2}\left(\frac{\partial S}{\partial \vec{x}}\right)^{2}+V(\vec{x})=0 .
$$

Taking two derivatives with respect to the components $x_{i}$ and $x_{j}$, one obtains (summation convention implied)

$$
\left(\frac{\partial}{\partial t}+\vec{p}(t, \vec{x}) \vec{\nabla}\right) u_{i j}(t, \vec{x})+u_{i k}(t, \vec{x}) u_{k j}(t, \vec{x})+\frac{\partial^{2}}{\partial x_{i} \partial x_{j}} V(t, \vec{x})=0
$$

where $u_{i j}=\partial S / \partial x_{i} \partial x_{j}$ is the curvature of the action, as introduced in sec. 2.1. Note that when one introduces characteristics, $d \vec{x} / d t=\vec{p}$, this is just the velocity field, and the term in brackets on the left-hand side in eq. (3.1) is just the convective derivative, which allows to switch from the partial derivatives to total derivatives and therefore from an Eulerian to a Lagrangian framework [69,70]. So in the Lagrangian view, i.e. along the characteristics, (3.1) becomes

$$
\frac{d}{d t} u_{i j}(t)+u_{i k}(t) u_{k j}(t)+\frac{\partial^{2}}{\partial x_{i} \partial x_{j}} V(t, \vec{x})=0
$$

or in quasi-2D [71-74]

$$
\frac{d}{d t} u(t)+u^{2}(t)+\frac{\partial^{2}}{\partial y^{2}} V(t, y(t))=0
$$

where we now call the spatial coordinate $y$, since the time $t$ will play, in a quasi-2D model, the role of the spatial coordinate $x$. 


\subsection{Derivation of the Fokker-Planck equation}

In order to derive a FPE for the probability density of the curvature, we approximate the random potential as seen by the particle in the $x$ or $t$-direction as white noise, since it moves fast in this direction compared to the transverse one. We therefore write eq. (3.3) as

$$
\frac{d u(t)}{d t}=-u^{2}(t)-\frac{D}{2} \Gamma(t)
$$

where $\Gamma(t)$ is a random function with correlation function

$$
c\left(t, t^{\prime}\right)=\left\langle\Gamma(t) \Gamma\left(t^{\prime}\right)\right\rangle=\delta\left(t-t^{\prime}\right)
$$

and $D$ is chosen in order to keep the integral of the correlation function constant. This will be discussed in more detail when considering related stochastic equations in chap. 5. $D$ is given by $[72,73,75]$

$$
D=\left.\int_{-\infty}^{\infty} \frac{\partial^{4}}{\partial y^{4}} c(x, y)\right|_{y=0} d x .
$$

and we can now proceed to derive the drift and diffusion constants of the FPE. From eqs. (B.6) from appendix B, we obtain

$$
\begin{aligned}
& D^{(1)}=-u^{2} \\
& D^{(2)}=D / 2
\end{aligned}
$$

such that the FPE (eq. (B.4) is given by

$$
\frac{\partial}{\partial t} P(u, t)=\left[\frac{\partial}{\partial u} u^{2}+\frac{\partial^{2}}{\partial u^{2}} \frac{D}{2}\right] P(u, t)
$$

We proceed by calculating the mean time to the first caustic, based on eq. (3.6).

\subsection{First caustic theory}

When is the first caustic encountered, i.e. when does the solution to eq. (3.6) become infinite for the first time? This requires solving eq. (3.6) with appropriate boundary conditions, which can be phrased in terms of a standard mean first passage time calculation (e.g. $[76-78])$ :

First, we need to analyze what happens at the boundaries of $u= \pm \infty$. It is instructive to look at the curvature equation without the random potential first. Setting $D=0$ in eq. (3.4), we can solve the equation by separation of variables to yield

$$
u(t)=\left(t+1 / u_{0}\right)^{-1}
$$



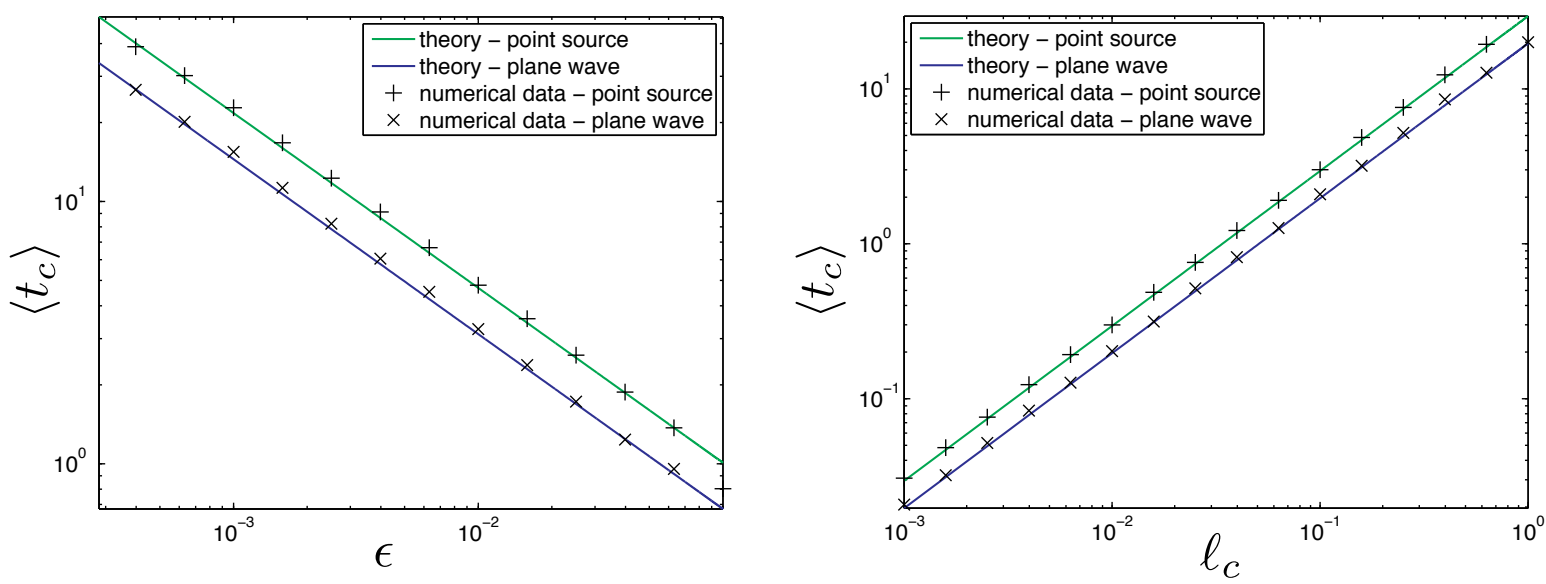

Figure 3.1: Test of the results of eqs. 3.10 for a Gaussian random potential and for a range of parameters of the random potential, $\epsilon$ and $\ell_{c}$. The average is taken over 50 realizations of the random potential, with 1000 trajectories simulated in each realization. The simulations match the theory well.

from which we observe that if $u_{0}$ is negative, $u(t)$ will approach $-\infty$ in the finite time $t=-1 / u_{0}$, from which it will reemerge with $+\infty$. A positive curvature will simply tend to flatten out over time $(u \rightarrow 0)$. A random potential can make the process diffuse from a positive curvature to a negative one, from where it will quickly approach a caustic [75]. We can now set up the boundary condition for the mean first passage problem of how long it takes to reach a caustic for the first time. In order to obtain the probability of this happening, we ask the inverse question: What is the probability of not having a singular point until $t$, at which $u \rightarrow-\infty$. For this, we classify the boundary $u=-\infty$ as an exit boundary and $u=\infty$ as an entrance boundary in the terminology of Feller [78,79]. This requires the boundary conditions

$$
\begin{aligned}
& P\left(u, t \mid u_{0}\right) \rightarrow 0 \quad \text { for } \quad u \rightarrow-\infty \\
& J(u, t) \rightarrow 0 \text { for } u \rightarrow+\infty
\end{aligned}
$$

where $J(u, t)$ is the probability flow (cf. appendix $\mathrm{B}$ ), which for eq. (3.6) is given by

$$
J(u, t)=\left[u^{2}-\frac{\partial}{\partial u} \frac{D}{2}\right] P(u, t) .
$$

What is now the probability of no singularity appearing until $t$ and the process stopping there? Assuming that $P\left(u, t \mid u_{0}\right)$ satisfies the boundary conditions above, this is given by

$$
G\left(t \mid u_{0}, t_{0}\right)=\int_{-\infty}^{+\infty} d u P\left(u, t \mid u_{0}\right)
$$


and the probability of a focus appearing at $t$ is the $1-G$. The probability density of this happening, $p_{f}$, is given by

$$
p_{f}\left(t \mid u_{0}\right)=-\frac{\partial}{\partial t} \int_{-\infty}^{\infty} d u P\left(u, t \mid u_{0}\right)
$$

which itself satisfies the backward FPE (cf. appendix B):

$$
\frac{\partial}{\partial t} p_{f}=-u_{0}^{2} \frac{\partial}{\partial u_{0}} p_{f}+\frac{\partial^{2}}{\partial u_{0}^{2}} p_{f} \quad \lim _{t \rightarrow 0, t \rightarrow \infty} p_{f}=0
$$

The average time during which $u_{0}$ goes to $u \rightarrow-\infty$ is called $t_{c}$ and is given by

$$
\left\langle t_{c}\left(u_{0}\right)\right\rangle=\int_{0}^{\infty} d t t p_{f}\left(t \mid u_{0}\right)
$$

To get an equation for $\left\langle t_{c}\left(u_{0}\right)\right\rangle$, we multiply $(3.9)$ by $t$ and integrate. The right hand side does not contain derivatives w.r.t. $t$ and is trivial. The left hand side gives

$$
\int_{0}^{\infty} d t t \frac{\partial}{\partial t} p_{f}\left(t \mid u_{0}\right)=\left.t p_{f}\left(t \mid u_{0}\right)\right|_{0} ^{\infty}-\int_{0}^{\infty} d t p_{f}\left(t \mid u_{0}\right)=0-1=-1
$$

and therefore the whole equation becomes

$$
-1=-u_{0}^{2} \frac{d}{d u_{0}}\left\langle t_{c}\left(u_{0}\right)\right\rangle+\frac{d^{2}}{d u_{0}}\left\langle t_{c}\left(u_{0}\right)\right\rangle, \quad \lim _{u_{0} \rightarrow-\infty}\left\langle t_{c}\left(u_{0}\right)\right\rangle=0, \lim _{u_{0} \rightarrow \infty}\left\langle t_{c}\left(u_{0}\right)\right\rangle=\text { finite. }
$$

This equation can be integrated twice as follows (see also [80]): Consider $u_{0}=x$ and $y=d\left(\left\langle t_{c}\left(u_{0}\right)\right\rangle / d u_{0}\right.$, then we first solve

$$
\frac{1}{2} y^{\prime}=x^{2} y-1
$$

which is a first-order, linear (in $y$ ) equation with the solution

$$
y=C e^{2 x^{3} / 3}-e^{2 x^{3} / 3} \int e^{-2 x^{3} / 3} d x .
$$

We set $C=0$ because we want $y$ to remain finite as $x \rightarrow \infty$. We now integrate $y$ to get $\left\langle t_{c}\left(u_{0}\right)\right\rangle$ and transform back to our original problem to obtain

$$
\left\langle t_{c}\left(u_{0}\right)\right\rangle=\frac{2}{D} \int_{-\infty}^{u_{0}} e^{2 \epsilon^{3} / 3 D} \int_{\epsilon}^{\infty} e^{-2 \eta^{3} / 3 D} d \eta d \epsilon .
$$

This can the be evaluated at $\infty$ or 0 to give a numerical value for the mean distance to reach the first focus from a point source or a plane wave source, respectively. It is given by $[73,75]$

$$
\left\langle t_{c}(\infty)\right\rangle=6.27 D^{-1 / 3} \quad \text { and } \quad\left\langle t_{c}(0)\right\rangle=4.18 D^{-1 / 3} \text {. }
$$




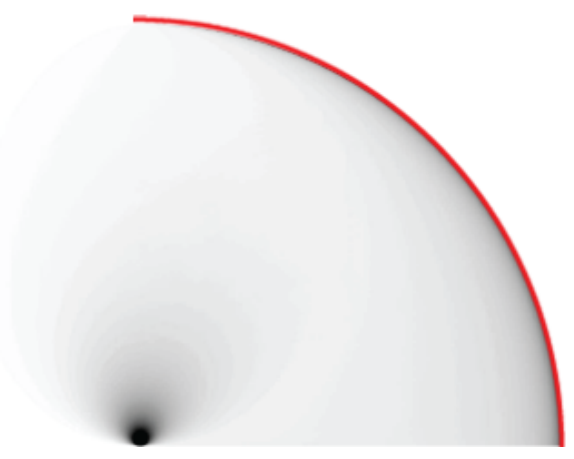

Figure 3.2: Flow emerging from a point source in a constant magnetic field pointing into the plane. The cyclotron orbits are enveloped by a circular caustic (red).

The results of eqs. 3.10 have not been tested numerically in detail. Rather, fully two-dimensional simulations confirming the validity of the quasi-2D approach have only been performed for special cases [72]. We therefore test the results of eqs. (3.10) for several correlation functions and a range of parameters of the random potential. The details of how the two-dimensional simulations are performed are given in sec. 3.6. We present some of the results of the simulations in fig. 3.1, and conclude that the quasi-2D approach is valid for a wide range of parameters of the random potential.

\subsection{Magnetic focusing}

We have seen how a random potential can focus trajectories and would now like to introduce a second, deterministic focusing mechanism. An important example is the deterministic focusing of charged particles by a constant magnetic field, which is called magnetic focusing, and which will play an important role in the experiment in the next chapter.

A constant magnetic field, pointing into the two-dimensional plane causes charged particles to move along cyclotron orbits, whose radius is inversely proportional to the magnetic field $B$ :

$$
r=\frac{m v}{q B}
$$

where we use units in which $m=q=v=1$. When the particles emerge from a point source, they form a circular caustic with twice the cyclotron radius, which envelopes the cyclotron orbits and which is the basic mechanism of magnetic focusing. We illustrate a flow from a point source in a constant magnetic field in fig. 3.2 

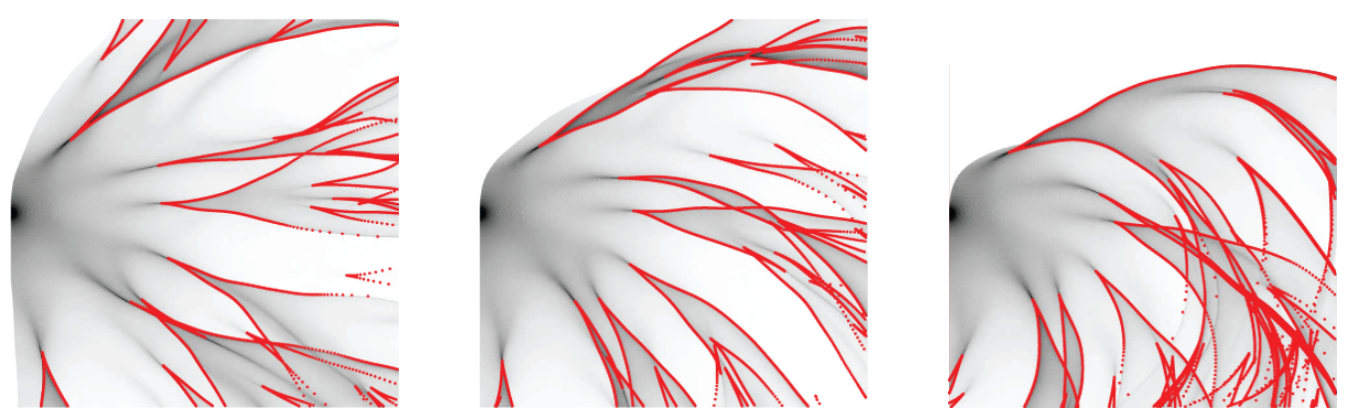

Figure 3.3: Particle flow from a point source for zero magnetic field and two increasing magnetic field strengths. Caustic locations are indicated in red.

\subsection{First caustics in a magnetic field}

We are now prepared to combine both focusing mechanisms, the random potential and the magnetic field. Three examples (for no magnetic field, and for two magnetic field strengths) are shown in fig. 3.3. Branching as well as the typical bending of the flow due to the magnetic field leading to magnetic focusing can be observed.

Including the magnetic field complicates the problem since we can now no longer identify one spatial direction with time and use the quasi-2D approximation as before. The solution to this problem is to find another variable which we can use to parametrize our equations. This is achieved by using polar coordinates $\{r, \varphi\}$ and by identifying the angular variable $\varphi$ with the time $t$. We can then look at small deviations from the circular motion of the particles, just as we looked at small lateral deviations from a straight line before. We again derive a curvature equation and perform a mean first passage time calculation.

The HJE in polar coordinates is

$$
\partial_{t} S+\frac{1}{2}\left(\partial_{r} S\right)^{2}+\frac{1}{2} \frac{1}{r^{2}}\left(\partial_{\varphi} S\right)^{2}+V(r, \varphi)=0 .
$$

The angular frequency of the charged particles is given by $\dot{\varphi}=v / r=\omega=q B / m$ which is just $B$ in our units. The momentum conjugate to $\varphi, p_{\varphi}$, is given by $p_{\varphi}=r^{2} \dot{\varphi}=r^{2} B$. Therefore, our new HJE, in which we can identify $t$ with $\varphi$, is given by

$$
\partial_{t} S+\frac{1}{2}\left(\partial_{r} S\right)^{2}+\frac{1}{2} r^{2} B^{2}+V(r(t))=0 .
$$

Taking two derivatives with respect to $r$ and evaluating the equation for the curvature $u=\partial_{r r} S$ along the characteristics, we obtain the following form of the curvature equation:

$$
\frac{d}{d t} u+u^{2}+B^{2}+\partial_{r r} V(r)=0
$$

We can now again derive a FPE equation. It is given by

$$
\frac{\partial}{\partial t} p(t, u)=\frac{\partial}{\partial u}\left(u^{2}+B^{2}\right) p(t, u)+\frac{D}{2} \frac{\partial^{2}}{\partial u^{2}} p(u, t)
$$


with $D$ still given by eq. (3.5). We derive from this, following the steps outlined above, the mean time to the first caustic starting from an initial curvature $u_{0}$ which is given by

$$
\left\langle t_{c}\left(u_{0}\right)\right\rangle=\frac{2}{D} \int_{-\infty}^{u_{0}} e^{2 / D\left(\epsilon^{3} / 3+\epsilon B^{2}\right)} \int_{\epsilon}^{\infty} e^{-2 / D\left(\eta^{3} / 3+\eta B^{2}\right)} d \eta d \epsilon .
$$

The expression can be simplified using the following transformation, which turns the variable integral limit of the second integral into a constant one:

$$
\begin{aligned}
\epsilon & =\frac{1}{\sqrt{2}}\left(\epsilon^{\prime}-\eta^{\prime}\right) \\
\eta & =\frac{1}{\sqrt{2}}\left(\epsilon^{\prime}+\eta^{\prime}\right) .
\end{aligned}
$$

We then obtain

$$
\left\langle t_{c}\left(u_{0}\right)\right\rangle=\frac{2}{D} \int_{-\infty}^{u_{0}} d \eta^{\prime} \int_{-\infty}^{\infty} d \epsilon^{\prime} e^{\sqrt{2} /(3 D)\left(6 \eta^{\prime} B^{2}+3 \eta^{\prime} \epsilon^{\prime 2}+\eta^{\prime}\right)}
$$

from which the Gaussian integral in $\epsilon^{\prime}$ can now be performed. For the case of a point source $\left(u_{0}=\infty\right)$, we can give an explicit expression in terms of Airy functions,

$$
\left\langle t_{c}(\infty)\right\rangle=\pi^{2}\left(\frac{2}{D}\right)^{1 / 3}\left(\mathrm{Ai}\left[-\left(\frac{2}{D}\right)^{2 / 3} B^{2}\right]^{2}+\mathrm{Bi}\left[-\left(\frac{2}{D}\right)^{2 / 3} B^{2}\right]^{2}\right)
$$

where $\mathrm{Ai}$ and $\mathrm{Bi}$ are the Airy functions of the first and second kind, respectively [81].

We confirm that our results give the expected values in the two limiting cases of no magnetic field and of no potential. The expression for the mean time until a caustic is reached from a point source in the limit as $B \rightarrow 0$ is given by

$$
\left\langle t_{c}(\infty)\right\rangle=\pi^{2} 2^{1 / 3}\left(\mathrm{Ai}[0]^{2}+\mathrm{Bi}[0]^{2}\right) D^{-1 / 3}=6.27 D^{-1 / 3}
$$

which reproduces the familiar result from eq. (3.10). For the case of the random potential disappearing, i.e. $D \rightarrow 0$, we calculate the limit by using approximations of the Airy functions for large negative values of their arguments from [81], and by using the shorthand $\alpha=(2 / D)^{1 / 3}$ :

$$
\begin{aligned}
\lim _{D \rightarrow 0}\left\langle t_{c}(\infty)\right\rangle & =\lim _{\alpha \rightarrow \infty} \pi^{2} \alpha\left(\sin \left[\frac{2}{3}(\alpha B)^{4 / 3}+\frac{\pi}{4}\right]^{2}+\cos \left[\frac{2}{3}(\alpha B)^{4 / 3}+\frac{\pi}{4}\right]^{2}\right) /(\pi \alpha B) \\
& =\pi / B
\end{aligned}
$$

which is the expected result, since without the random potential, a particle has to travel half a circle of radius $1 / B$ to reach a caustic.

We plot our solution, eq. (3.11), for different magnetic fields and varying parameters $\epsilon$ and $\ell_{c}$ of the correlation function of the random potential in fig. 3.4, and compare it to numerical simulations. The simulations are again fully two-dimensional in order to test the validity of the quasi-2D approach used for the analytical solution, and we observe that they coincide with the analytical prediction. 


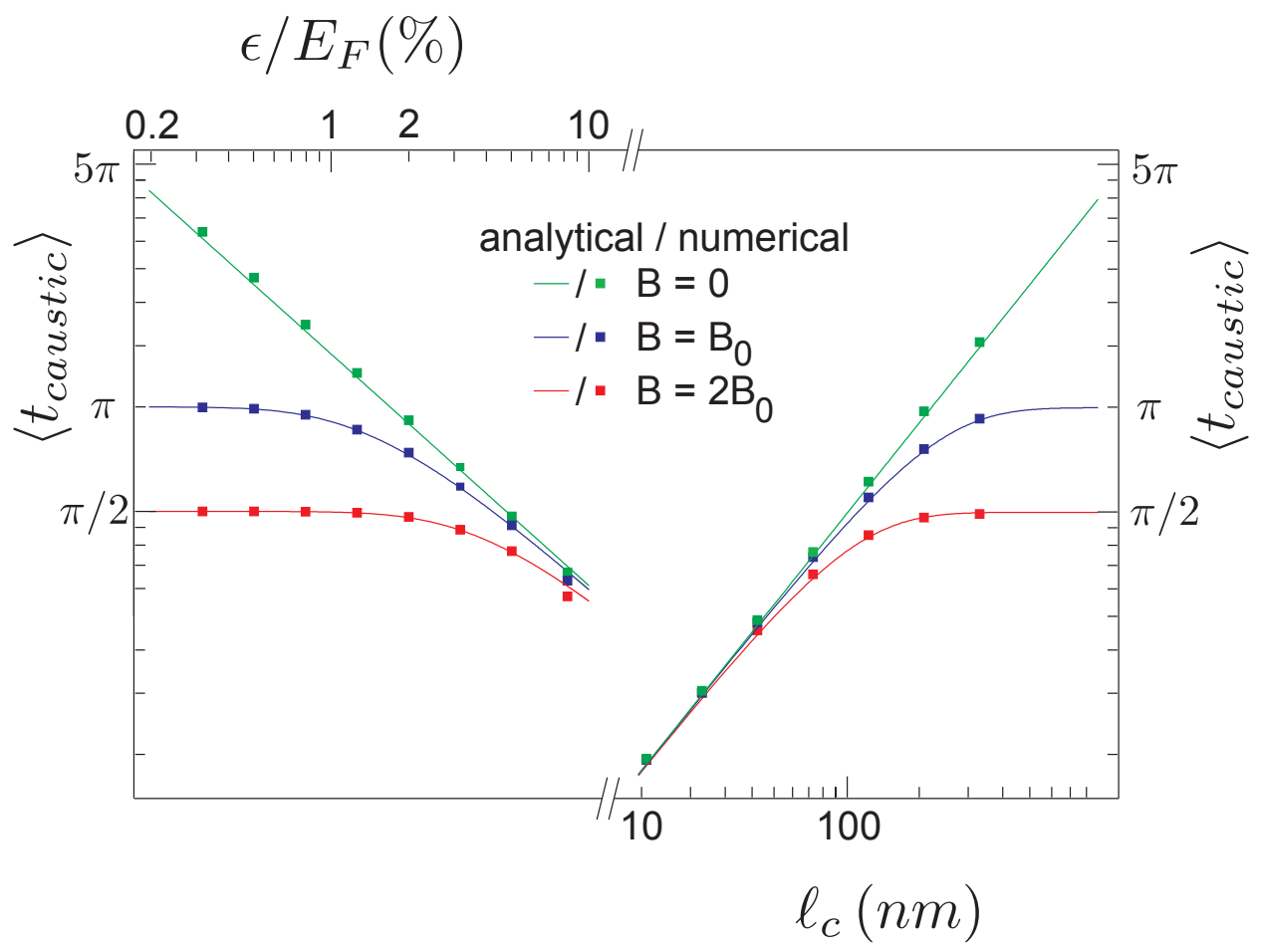

Figure 3.4: Scaling of the mean time (or distance) of the first caustic along a trajectory as a function of the two parameters of the random potential and for different values of the magnetic field. For very weak potentials (small $\epsilon$ or large $\ell_{c}$ ), the focal time approaches that of the deterministic magnetic focusing. Numerical values obtained from a fully two-dimensional simulation (see sec. 3.6 for details) match the theoretical results well. 


\subsubsection{Higher moments}

Until now, we have only calculated the mean time to a caustic. Here, we also describe a method to calculate the higher moments of the caustic distribution. We demonstrate the method by calculating the second moment, however, all other moments can in principle be calculated (up to an integral) by the same method. By choosing an appropriate coordinate system, the limits of the integrals, which now depend on the integration variables, can be transformed to constant values, making a numerical integration possible. The second moment is given by $[76,78]$

$$
\begin{aligned}
\left\langle t_{c}\left(u_{0}\right)^{2}\right\rangle= & 8 \int_{-\infty}^{u_{0}} d \alpha e^{2 / 3\left(\alpha^{3}+3 \alpha B\right)} \int_{\alpha}^{\infty} d \beta e^{-2 / 3\left(\beta^{3}+3 \beta B\right)} \\
& \times \int_{-\infty}^{\beta} d \epsilon e^{2 / 3\left(\epsilon^{3}+3 \epsilon B\right)} \int_{\epsilon}^{\infty} d \eta e^{-2 / 3\left(\eta^{3}+3 \eta B\right)} .
\end{aligned}
$$

We can eliminate the variable integration limits recursively using the transformation

$$
\mathbf{T}=\left(\begin{array}{ll}
1 & 0 \\
1 & 1
\end{array}\right)
$$

(note that the Jacobian of the transformation is still unity) such that the old variables are related to the new ones as

$$
\left(\begin{array}{c}
\epsilon \\
\eta
\end{array}\right)=\mathbf{T}\left(\begin{array}{c}
\epsilon^{\prime} \\
\eta^{\prime}
\end{array}\right), \quad\left(\begin{array}{c}
\beta \\
\epsilon^{\prime}
\end{array}\right)=\mathbf{T}\left(\begin{array}{c}
\beta^{\prime} \\
\epsilon^{\prime \prime}
\end{array}\right), \quad\left(\begin{array}{c}
\alpha \\
\beta^{\prime}
\end{array}\right)=\mathbf{T}\left(\begin{array}{c}
\alpha^{\prime} \\
\beta^{\prime \prime}
\end{array}\right)
$$

and so

$$
\begin{gathered}
\alpha=\alpha^{\prime} \\
\beta=\beta^{\prime}=\alpha^{\prime}+\beta^{\prime \prime} \\
\epsilon=\epsilon^{\prime}=\beta^{\prime}+\epsilon^{\prime \prime}=\alpha^{\prime}+\beta^{\prime \prime}+\epsilon^{\prime \prime} \\
\eta=\eta^{\prime}+\epsilon^{\prime}=\eta^{\prime}+\alpha^{\prime}+\beta^{\prime \prime}+\epsilon^{\prime \prime} \\
\left\langle t_{c}\left(u_{0}\right)^{2}\right\rangle=8 \int_{-\infty}^{u_{0}} d \alpha^{\prime} \int_{0}^{\infty} d \beta^{\prime \prime} \int_{-\infty}^{0} d \epsilon^{\prime \prime} \int_{0}^{\infty} d \eta^{\prime} \\
\times e^{2 / 3\left(\alpha^{\prime 3}-\left(\alpha^{\prime}+\beta^{\prime \prime}\right)^{3}+\left(\alpha^{\prime}+\beta^{\prime \prime}+\epsilon^{\prime \prime}\right)^{3}-\left(\alpha^{\prime}+\beta^{\prime \prime}+\epsilon^{\prime \prime}+\eta^{\prime}\right)^{3}-3 B\left(\beta^{\prime \prime}+\eta^{\prime}\right)\right)}
\end{gathered}
$$

which can now be integrated numerically, e.g. using a Monte Carlo method.

\subsection{D equations and numerics for the curvature equation}

We have studied the statistics of random caustics analytically in a quasi-2D model in order to describe a two-dimensional system with one-dimensional equations. To test 
the validity of this approximation, we perform extensive numerical simulations in two dimensions, which allow us to obtain statistics on the location of caustics. This requires setting up the two-dimensional equations for the curvature with their associated initial conditions. We will first perform the calculations without the magnetic field, and introduce it into the $2 \mathrm{D}$ equations in sec. 3.7.

Since the curvature matrix $\mathbf{U}$ with elements $u_{i j}$ is symmetric, we can always diagonalize it using a rotation matrix $\mathbf{R}$. In two dimensions, this gives

$$
\mathbf{R}^{\mathbf{T}} \mathbf{U} \mathbf{R}=\left(\begin{array}{cc}
\cos \theta & \sin \theta \\
-\sin \theta & \cos \theta
\end{array}\right)\left(\begin{array}{cc}
u_{11} & u_{12} \\
u_{21} & u_{22}
\end{array}\right)\left(\begin{array}{cc}
\cos \theta & -\sin \theta \\
\sin \theta & \cos \theta
\end{array}\right)=\left(\begin{array}{cc}
\lambda_{1} & 0 \\
0 & \lambda_{2}
\end{array}\right)=\boldsymbol{\Lambda} .
$$

The eigenvalues $\lambda_{1,2}$ are the two principal curvatures of the action. When one of the eigenvalues becomes infinite (its inverse goes through zero), the trajectory has touched a caustic. Numerically, we simulate the equations for $\lambda_{1}, \lambda_{2}$ and the diagonalization parameter $\theta$, which are then solved together with the equations of motion of the trajectory. The differential equations for these can be derived by taking the derivative of eq. (3.12) and inserting eq. (3.2). We also define $F_{i j}=\partial^{2} V /\left(\partial x_{i} \partial x_{j}\right)$ and obtain [73]

$$
\begin{aligned}
\frac{d}{d t} \lambda_{1} & =-\lambda_{1}^{2}+F_{11} \cos ^{2} \theta+F_{22} \sin ^{2} \theta+F_{12} \sin 2 \theta \\
\frac{d}{d t} \lambda_{2} & =-\lambda_{2}^{2}+F_{22} \cos ^{2} \theta+F_{11} \sin ^{2} \theta-F_{12} \sin 2 \theta \\
\frac{d}{d t} \theta & =\frac{1}{\lambda_{1}-\lambda_{2}}\left[\left(F_{22}-F_{11}\right) \sin 2 \theta+F_{12} \cos 2 \theta\right] .
\end{aligned}
$$

\subsubsection{Initial Conditions}

In order to study the curvature equation and its statistics numerically, we need to supply it with initial conditions. The two most important ones are the plane wave and the point source initial condition. Together with requiring all trajectories to have the same total energy, one can uniquely determine the initial curvature matrix.

\subsubsection{Plane Wave}

We first derive an equation for the plane wave initial condition, i.e. the matrix elements $u_{i j}(0)=u_{i j}^{0}$. We assume two trajectories very close to each other, with phase space coordinates

$$
\begin{aligned}
y^{\prime \prime} & =y^{\prime}+\frac{1}{2} F_{y}(\Delta t)^{2} \\
x^{\prime \prime} & =x^{\prime}+p_{x}^{\prime} \Delta t \\
p_{x}^{\prime \prime} & =p_{x}^{\prime}+F_{x} \Delta t \\
p_{y}^{\prime \prime} & =p_{y}^{\prime}+F_{y} \Delta t
\end{aligned}
$$




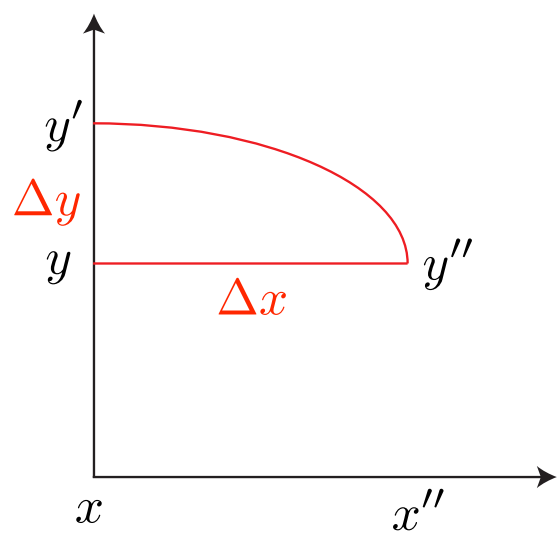

Figure 3.5: Geometry of the plane wave initial condition with a slightly displaced ray at $y^{\prime}$. Together with the condition that both rays must have the same energy the initial conditions for the curvature matrix can be derived.

where the primed and unprimed quantities are illustrated in fig. 3.5. We introduce the notation $y^{\prime}-y=\Delta y=\frac{1}{2} F_{y}(\Delta t)^{2}$ and $x^{\prime \prime}-x=\Delta x=p_{x}^{\prime} \Delta t$. Also $p_{x}^{\prime 2}-p_{x}^{2}=F_{y} \Delta y$, such that

$$
\begin{gathered}
\Delta x=p_{x}^{\prime} \Delta t=\sqrt{2 F_{y} \Delta y+p_{x}^{2}} \Delta t \\
u_{12}^{0}=\frac{\Delta p_{x}}{\Delta y}=\frac{p_{x}^{\prime}-p_{x}}{\Delta y}=\frac{\sqrt{p_{x}^{2}+2 F_{y} \Delta y}-p_{x}}{\Delta y} \approx \frac{p_{x}+\frac{F_{y} \Delta y}{p_{x}}-p_{x}}{\Delta y}=\frac{F_{y}}{p_{x}} \\
u_{21}^{0}=u_{12}^{0}=\frac{\Delta p_{y}}{\Delta x}=\frac{p_{y}^{\prime \prime}-p_{y}}{\Delta x}=\frac{\frac{F_{y} \Delta x}{p_{x}}}{\Delta x}=\frac{F_{y}}{p_{x}} \\
u_{11}^{0}=\frac{\Delta p_{x}}{\Delta x}=\frac{p_{x}^{\prime \prime}-p_{x}}{\Delta x}=\frac{p_{x}+F_{x} \Delta t-p_{x}}{\Delta x}=\frac{F_{x} \Delta t}{\Delta x}=\frac{F_{x}}{p_{x}}
\end{gathered}
$$

and $u_{22}^{0}=0$ by definition of the plane wave. So the initial conditions for the elements of the curvature equation are

$$
u_{i j}^{0}=\left(\begin{array}{cc}
\frac{F_{x}}{p_{x}} & \frac{F_{y}}{p_{x}} \\
\frac{F_{y}}{p_{x}} & 0
\end{array}\right)
$$

which can be diagonalized by $\mathbf{R}^{\mathbf{T}} \mathbf{U} \mathbf{R}=\boldsymbol{\Lambda}$.

A special case is $F_{x}=0$. In this case, the matrix is diagonalized using an angle of $\theta=\pi / 4$ and yields

$$
\boldsymbol{\Lambda}=\left(\begin{array}{cc}
\frac{F_{y}}{p_{x}} & 0 \\
0 & -\frac{F_{y}}{p_{x}}
\end{array}\right)
$$

Otherwise the diagonalization is more general and the angle is given by

$$
\theta=\tan ^{-1}\left(\frac{1}{2}\left(-\alpha \pm \sqrt{\alpha^{2}+4}\right)\right)
$$


where $\alpha=\left(u_{11}^{0}-u_{22}^{0}\right) / u_{12}^{0}=\frac{u_{11}^{0}}{u_{12}^{0}}=\frac{F_{x}^{0}}{F_{y}^{0}}$, while the eigenvalues are

$$
\begin{aligned}
& \lambda_{1}^{0}=u_{11}^{0} \cos ^{2} \theta+u_{12}^{0} \sin 2 \theta \\
& \lambda_{2}^{0}=u_{11}^{0} \sin ^{2} \theta-u_{12}^{0} \sin 2 \theta .
\end{aligned}
$$

\subsubsection{Point Source}

For the point source, we go to polar coordinates, establish the matrix $\tilde{u}_{i j}$, and transform it back to $u_{i j}$. In the new polar coordinate system $(r, \varphi)$,

$$
\tilde{u}_{i j}^{0}=\left(\begin{array}{cc}
\frac{F_{r}}{p_{r}} & \frac{F_{\varphi}}{p_{r}} \\
\frac{F_{\varphi}}{p_{r}} & 0
\end{array}\right)
$$

and we can transform the individual components back using the chain rule:

$$
\begin{aligned}
\frac{\partial S}{\partial x} & =\frac{\partial S}{\partial r} \frac{\partial r}{\partial x}+\frac{\partial S}{\partial \varphi} \frac{\partial \varphi}{\partial x} \\
u_{11}^{0}=\frac{\partial^{2} S}{\partial x^{2}} & =\frac{\partial^{2} S}{\partial r^{2}}\left(\frac{\partial r}{\partial x}\right)^{2}+2 \frac{\partial^{2} S}{\partial r \partial \varphi} \frac{\partial \varphi}{\partial x} \frac{\partial r}{\partial x}+\frac{\partial^{2} S}{\partial \varphi^{2}}\left(\frac{\partial \varphi}{\partial x}\right)^{2}+\frac{\partial S}{\partial r} \frac{\partial^{2} r}{\partial x^{2}}+\frac{\partial S}{\partial \varphi} \frac{\partial^{2} \varphi}{\partial x^{2}} .
\end{aligned}
$$

The last term vanishes because $p_{\varphi}=\partial S / \partial \varphi=0 . u_{12}^{0}$ and $u_{22}^{0}$ are calculated analogously, the result being

$$
\begin{aligned}
& u_{11}^{0}=\tilde{u}_{11} \cos ^{2} \varphi-\tilde{u}_{12} \frac{\sin 2 \varphi}{r}+p_{r} \frac{\sin ^{2} \varphi}{r}+\frac{p_{\varphi}}{r^{2}} \sin 2 \varphi \\
& u_{12}^{0}=\frac{\tilde{u}_{11}}{2} \sin 2 \varphi+\tilde{u}_{12} \frac{\cos 2 \varphi}{r}-p_{r} \frac{\sin 2 \varphi}{2 r}-\frac{p_{\varphi}}{r^{2}} \cos 2 \varphi \\
& u_{22}^{0}=\tilde{u}_{11} \sin ^{2} \varphi+\tilde{u}_{12} \frac{\sin 2 \varphi}{r}+p_{r} \frac{\cos ^{2} \varphi}{r}+\frac{p_{\varphi}}{r^{2}} \sin 2 \varphi .
\end{aligned}
$$

The matrix $u_{i j}^{0}$ is now diagonalized by a rotation with angle $\theta$ as given by eq. (3.15). We first calculate

$$
\alpha=\frac{u_{11}^{0}-u_{22}^{0}}{u_{12}}=\frac{-2 \tilde{u}_{12}^{0} \sin 2 \varphi-p_{r} \cos 2 \varphi+r \tilde{u}_{11}^{0} \cos 2 \varphi}{\tilde{u}_{12}^{0} \cos 2 \varphi-\frac{p_{r}}{2} \sin 2 \varphi+r \tilde{u}_{11}^{0} \sin 2 \varphi}
$$

and then perform the limit $r \rightarrow 0$, which yields the initial condition:

$$
\lim _{r \rightarrow 0} \alpha=\frac{-2 \tilde{u}_{12}^{0} \sin 2 \varphi-p_{r} \cos 2 \varphi}{\tilde{u}_{12}^{0} \cos 2 \varphi-\frac{p_{r}}{2} \sin 2 \varphi}
$$

and also $\tilde{u}_{12}^{0}=\frac{F_{\varphi}}{p_{r}}$ with $p_{r}$ being some nonzero number proportional to the square root of the energy (minus the potential) at the source. $F_{\varphi}$, however, is the torque and therefore proportional to $r$ and so vanishes at $r=0$. Then the limit can be evaluated as

$$
\lim _{r \rightarrow 0} \alpha=\frac{2}{\tan 2 \varphi} .
$$


Inserting this into eq. 3.15 yields

$$
\theta=\varphi
$$

So in order to diagonalize the coordinate system we have to rotate the system in the direction the trajectory is moving. A corresponding calculation shows that the initial eigenvalues $\lambda_{1,2}^{0}$ are (assuming $p_{\varphi}=0$ and $r \rightarrow 0$ )

$$
\begin{aligned}
\lambda_{1}^{0} & =\tilde{u}_{11}^{0}=\frac{F_{r}}{p_{r}}=F_{x} \cos \theta+F_{y} \sin \theta \\
\lambda_{2}^{0} & =\frac{p_{r}}{r}=\infty
\end{aligned}
$$

\subsubsection{Inverse equations}

Inverse equations are needed when there is a caustic, since one of the eigenvalues explodes. Before this happens, the relevant equation is inverted and the solution moving through a zero then indicates the appearance of a caustic. In particular, the inverse equation for $\lambda_{2}$ has to be used initially for the point source, since this is nothing but a caustic. The inverse equations are:

$$
\begin{aligned}
& \frac{d}{d t} \kappa_{1}=1-\kappa_{1}^{2}\left(F_{11} \cos ^{2} \theta+F_{22} \sin ^{2} \theta+F_{12} \sin 2 \theta\right) \\
& \frac{d}{d t} \kappa_{2}=1-\kappa_{2}^{2}\left(F_{11} \sin ^{2} \theta+F_{22} \cos ^{2} \theta-F_{12} \sin 2 \theta\right) .
\end{aligned}
$$

\subsection{Curvature equation with magnetic field}

We now assume charged particles and extend the equations to include a magnetic field perpendicular to the two-dimensional plane in which the particles are moving. We change the curvature equation and include a vector potential with components $A_{i}$ (i.e. $\vec{p} \rightarrow(\vec{p}-q \vec{A}), p_{i} \rightarrow\left(p_{i}-q A_{i}\right)$, with charge $q=1$ ) to obtain (summation convention implied)

$$
\begin{aligned}
& \frac{d}{d t} u_{i j}(t)+u_{i k}(t) u_{k j}(t)+\left(\partial_{j} A_{k} \partial_{i} A_{k}\right)-\partial_{j} A_{k} u_{k i}-\partial_{i} A_{k} u_{k j}- \partial_{i j} A_{k}\left(\partial_{k} S-A_{k}\right) \\
&+\frac{\partial^{2}}{\partial x_{i} \partial x_{j}} V(t, \vec{x})=0 .
\end{aligned}
$$

We again look for the diagonalized $\Lambda$ such that

$$
\mathbf{R}^{\mathrm{T}} \mathbf{u R}=\Lambda
$$

with the rotation matrix

$$
\mathbf{R}=\left(\begin{array}{rr}
\cos \theta & -\sin \theta \\
\sin \theta & \cos \theta
\end{array}\right)
$$


and differentiate eq. 3.17) giving

$$
\left(\frac{d}{d t} \mathbf{D}^{\mathbf{T}}\right) \mathbf{u D}+\mathbf{D}^{\mathbf{T}}\left(\frac{d}{d t} \mathbf{u}\right) \mathbf{D}+\mathbf{D}^{\mathbf{T}} \mathbf{u}\left(\frac{d}{d t} \mathbf{D}\right)=\frac{d}{d t} \Lambda
$$

The new terms result from the middle term in eq. (3.18), in particular, from the modified $\left(\frac{d}{d t} \mathbf{u}\right)$. This is given by

$$
\frac{d}{d t} u_{l m}=-u_{l m} u_{i m}-C_{m i} C_{l i}+C_{m i} u_{l i}+C_{l i} u_{m i}
$$

where we have introduced the notation $\partial_{i} A_{k}=C_{i k}$. We treat the new terms involving the matrix $C_{i j}$ separately, using the Landau gauge $\vec{A}=(-y B, 0,0)$ :

$$
\begin{aligned}
-D_{j l}^{T} C_{m i} C_{l i} D_{m k} & =-D_{j 2}^{T}\left(C_{21}\right)^{2} D_{2 k}=-B^{2} D_{2 j} D_{2 k} \\
D_{j l}^{T} C_{m i} u_{l i} D_{m k} & =\Lambda_{j l} D_{l i}^{T} C_{m i} D_{m k}=-B\left[\Lambda_{j 1} D_{11} D_{2 k}+\Lambda_{j 2} D_{12} D_{2 k}\right] \\
D_{j l}^{T} C_{l i} u_{i m} D_{m k} & =D_{j l}^{T} C_{l i} D_{i m} \Lambda_{m k}=-B\left[\Lambda_{1 k} D_{11} D_{2 j}+\Lambda_{2 k} D_{12} D_{2 j}\right]
\end{aligned}
$$

The modifications for the equation for $\left\{\lambda_{1}, \lambda_{2}, \theta\right\}$ are obtained by setting $\{(j=k=$ $1),(j=k=2),(j=1, k=2)\}$ and then adding the three terms from eq. (3.19):

$$
\begin{aligned}
\lambda_{1}: & -B\left[B \sin ^{2} \theta+\lambda_{1} \sin 2 \theta\right] \\
\lambda_{2}: & -B\left[B \cos ^{2} \theta-\lambda_{2} \sin 2 \theta\right] \\
\theta: & -B\left[\frac{B}{2} \sin 2 \theta+\left(\lambda_{1} \cos ^{2} \theta-\lambda_{2} \sin ^{2} \theta\right)\right] .
\end{aligned}
$$

The final result for the differential equations for $\left\{\lambda_{1}, \lambda_{2}, \theta\right\}$ is then

$$
\begin{aligned}
\frac{d}{d t} \lambda_{1} & =-\lambda_{1}^{2}+F_{11} \cos ^{2} \theta+F_{22} \sin ^{2} \theta+F_{12} \sin 2 \theta-B^{2} \sin ^{2} \theta-B \lambda_{1} \sin 2 \theta \\
\frac{d}{d t} \lambda_{2} & =-\lambda_{2}^{2}+F_{22} \cos ^{2} \theta+F_{11} \sin ^{2} \theta-F_{12} \sin 2 \theta-B^{2} \cos ^{2} \theta+B \lambda_{2} \sin 2 \theta \\
\frac{d}{d t} \theta & =\frac{1}{\lambda_{1}-\lambda_{2}} \\
& {\left[\left(F_{22}-F_{11}\right) \sin 2 \theta+F_{12} \cos 2 \theta-\frac{B^{2}}{2} \sin 2 \theta-B\left(\lambda_{1} \cos ^{2} \theta-\lambda_{2} \sin ^{2} \theta\right)\right] . }
\end{aligned}
$$




\subsubsection{Initial Conditions}

With a magnetic field we need to alter our definition of the force (assuming no movement in the $z$-direction):

$$
\begin{aligned}
& H=\frac{1}{2}\left(p_{i}-A_{i}\right)^{2}+V=\frac{1}{2}\left(p_{x}+B y\right)^{2}+\frac{1}{2} p_{y}^{2}+V \\
& F_{x}=\dot{p}_{x}=-\frac{\partial H}{\partial x}=-\frac{\partial V}{\partial x} \\
& F_{y}=\dot{p}_{y}=-\frac{\partial H}{\partial y}=-\frac{\partial V}{\partial y}-B\left(p_{x}+B y\right)=-\frac{\partial V}{\partial y}-B v_{x}
\end{aligned}
$$

where $v_{x}$ is the velocity in the $x$-direction. Note that because of the choice $\vec{A}=$ $(-B y, 0,0)$, only the force in the $y$ direction is modified. So the new $u_{i j}$ with which one calculates $\left\{\lambda_{1}, \lambda_{2}, \theta\right\}$ is the same as 3.14 and 3.16 except with $F_{y}$ from 3.21 and $v_{i}$ instead of $p_{i}$.

\subsection{Full first caustic distribution}

Up to now, we have only been concerned with the mean time to the first caustic, i.e. the first moment of the caustic distribution function $P_{c}(t)$. An expression for the probability to reach the first caustic $P_{f c}(t)$ was derived from an equivalent formulation of the curvature equation by White and co-workers [71,72, 75, 82]. In our formulation of the problem, their approximate solution of $P_{f c}(t)$ for the case of an initially plane source is given by

$$
P_{f c}(t)=\left(\alpha^{2}(2 \pi D)^{-1 / 2} t^{-5 / 2}+C_{F} D^{1 / 3}\right) e^{-\lambda_{1} D^{1 / 3} t-\alpha^{4} /\left(6 D t^{3}\right)}
$$

where $D$ was defined in eq. 3.5 and $\alpha \approx 1.854, \lambda_{1} \approx 0.281, C_{F} \approx 0.314$. Eq. (3.22) approximates well the probability to reach the first caustic. However, we will also need the probability to reach any caustic. This is accomplished by observing that every caustic, at least for the formulation in terms of the curvature equation, acts like a point source. Therefore, after the first caustic has been reached, the probability to reach a further caustic quickly settles to a constant, which is the inverse of the average time to the first caustic from a point source. This asymptote was already given in [75]. Here, we use it to construct a combined solution for $P_{c}(t)$, the probability to reach any caustic. We note that this is not a probability distribution in the usual sense because it is not normalizable. It is given by

$$
P_{c}(t)= \begin{cases}\left(\alpha^{2}(2 \pi D)^{-1 / 2} t^{-5 / 2}+C_{F} D^{1 / 3}\right) e^{-\lambda_{1} D^{1 / 3} t-\alpha^{4} /\left(6 D t^{3}\right)} & \text { if } t \leq t_{1} \\ 1 /\left(6.27 D^{-1 / 3}\right) & \text { if } t>t_{1}\end{cases}
$$

where $t_{1}$ is the value at which $1 /\left(6.27 D^{-1 / 3}\right)$ is larger than the other expression, but only after the latter has peaked. We define

$$
t_{0}=6.27 D^{-1 / 3}
$$




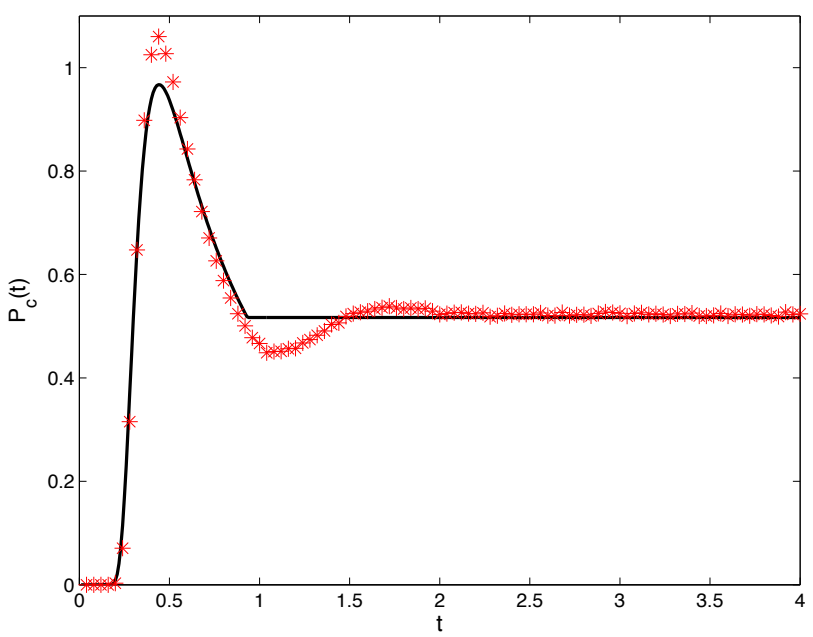

Figure 3.6: $P_{c}(t)$ analytic quasi 2D solution (black) and 2D numerical simulation for $\epsilon=8 \%$ of the particles' energy and $\ell_{c}=0.1$ (Gaussian correlation function).

since this is the mean time between two foci. It is emphasized, however, that this definition, which will become important in chapter 6, differs from the usual one as in [83] by a prefactor, which depends on the type of correlation function used.

We perform numerical simulations of the full two-dimensional problem and compare it to the quasi-2D analytics (eq. (3.23) ) in fig. 3.6. The numerical $P_{c}$ is slightly underestimated at the peak and overestimated due to our simple construction of $P_{c}(t)$. Apart from this, we obtain excellent agreement. We find that this holds true for a wide range of parameters and correlation functions. We also note curves for different parameters and correlation functions collapse onto one universal curve if the time axis is rescaled by $t_{0}$. This idea will be reused and extended in chapter 6 to calculate the density of branches transverse to the flow.

\subsection{Conclusion}

In this chapter, we have analyzed caustic statistics in a random potential along trajectories. We have tested existing results using detailed two-dimensional simulations, and have found that the simulations match well the theoretical predictions for a wide range of parameters and different correlation functions. We extended the theory to include as a second, deterministic focusing mechanism a constant magnetic field. We have shown how the two focusing processes compete in causing caustics, and we have again confirmed the validity of the quasi-2D theory using fully two-dimensional numerical simulations. We have described in detail how to perform the numerical simulations. This chapter also naturally leads to the next, which is an analysis of a magnetic focusing experiment in 
which both the magnetic field and a random potential play important roles. In addition to this, we have given an expression for the first caustic distribution valid for all times, which will be needed in chapter 6 


\section{Application: Magnetic Focusing}

In the previous chapter, we have studied the interplay of focusing of charged particles by a magnetic field and focusing by a random potential. The results obtained there will be applied to experimental data from a magnetic focusing experiment in this chapter. Magnetic focusing of charged particles has been widely used to study fundamental transport properties of semiconductor microstructures, in particular in two-dimensional electron gases (2DEGs) [84-90]. In these systems, electrons are confined in one spatial direction, but move almost freely in the other two dimensions, which means that their mean free path is much greater than the system size. The motion of the electrons is therefore ballistic, and can be described classically.

When studying transport in a 2DEG, quantum point contacts (QPCs) are usually used as emitters and receivers of the electrons. A QPC is a narrow constriction (with a width of the order of the Fermi wavelength of the electrons) in the electrostatic walls which define the geometry of the system. In an experiment, there are typically several point contacts, to which leads are attached. Electrons can flow into or out of the system through the contacts, and the charge transport through the system is treated as a scattering process within the Landauer-Büttiker formalism [91-93], which relates the conductance to the transmittance of the system. Magnetic focusing experiments are typically three or four-terminal devices. The resistance between the emitter and the receiver is obtained by dividing the voltage measured across these two contacts by the current which flows between the emitter and an additional contact. The resistance thus obtained translates directly into the transmission of electrons from the emitter to the receiver.

When a perpendicular magnetic field is applied, this can focus the electron flow as described in the previous chapter. When the magnetic field is such that it focuses the flow at the receiver, a lot of electrons will be transmitted from emitter to receiver, and therefore a large resistance will be measured.

In the standard magnetic focusing geometry the emitter and the collector are arranged along a line, which is also called transverse magnetic focusing (TMF). In this geometry, which is illustrated in fig. 4.1 a, focusing occurs when a multiple of the cyclotron diameter of the electrons matches the distance between the two QPCs. At the corresponding values of the magnetic field, a circular caustic with twice the diameter of the cyclotron diameter is located at the receiver, which will appear as a focusing peak in the resistance. These peaks are observed at multiples of $B_{0}$, which is the magnetic field at which the cyclotron diameter of the electrons is equal to the distance between emitter and receiver. 


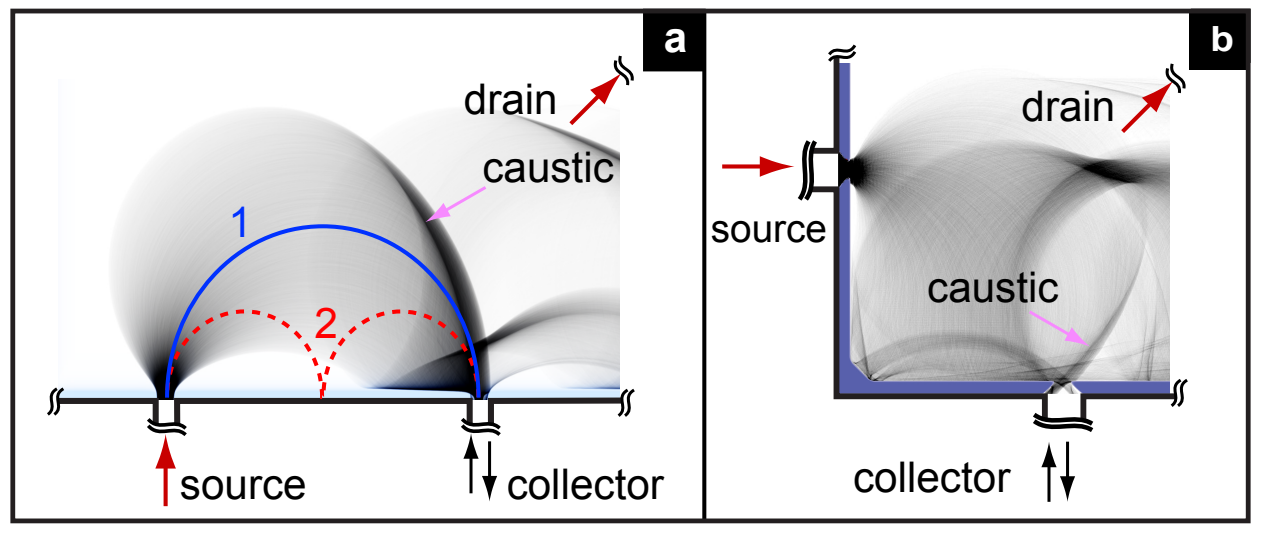

Figure 4.1: (a) Transverse magnetic focusing (TMF): Electrons flowing out of a quantum point contact are focused at the emitter at multiples of $B_{0}$, which is the magnetic field forcing centrally emitted particles on a half-circle between emitter and collector (trajectory 1 in blue). The next focusing occurs at $2 B_{0}$ (trajectory 2 in red). (b) Magnetic focusing in a corner device. The focusing structure is more complicated, for example there is an additional focusing at $\sqrt{2} B_{0}$, which is plotted here.

In contrast to TMF, the experiment analyzed here was performed in a corner-shaped geometry depicted in fig. 4.1 b. This geometry complicates the experiment in several ways, but also allows new insight into ballistic transport in semiconductor microstructures. For example, there is a focusing of the electron flow at a non-integer value of the magnetic field. Also, we will find that some peaks in the new device are more sensitive to random focusing by a disorder potential and that, even though the mean free path of the electrons is much larger than the system size, a strong influence of branching on the conductance properties of the device can be observed.

The chapter is organized in the following way: First, the experimental setup will be described and the experimental results will be presented. This is followed by a detailed analysis of the geometry and its effects on the measurements. We then consider the role of branching by a random potential and use the results from the previous chapter to show that the experimental data is a result of the focusing by the magnetic field, the focusing by a random potential, and the peculiarities of the geometry used here. The results obtained in collaboration with our experimental colleagues (D. Maryenko and J. Smet, MPI for Solid State Research, Stuttgart) are being prepared for publication [94].

\subsection{Experimental setup and results}

The experiment is performed on a modulation doped GaAs/AlGaAs heterostructure in which the 2DEG is located $150 \mathrm{~nm}$ underneath the crystal surface. Two types of devices are studied. One with a normal, sharp corner (type I), and the other with a chamfered corner (type II). The two types are shown in the inset of fig. 4.2 a and c, respectively. The 

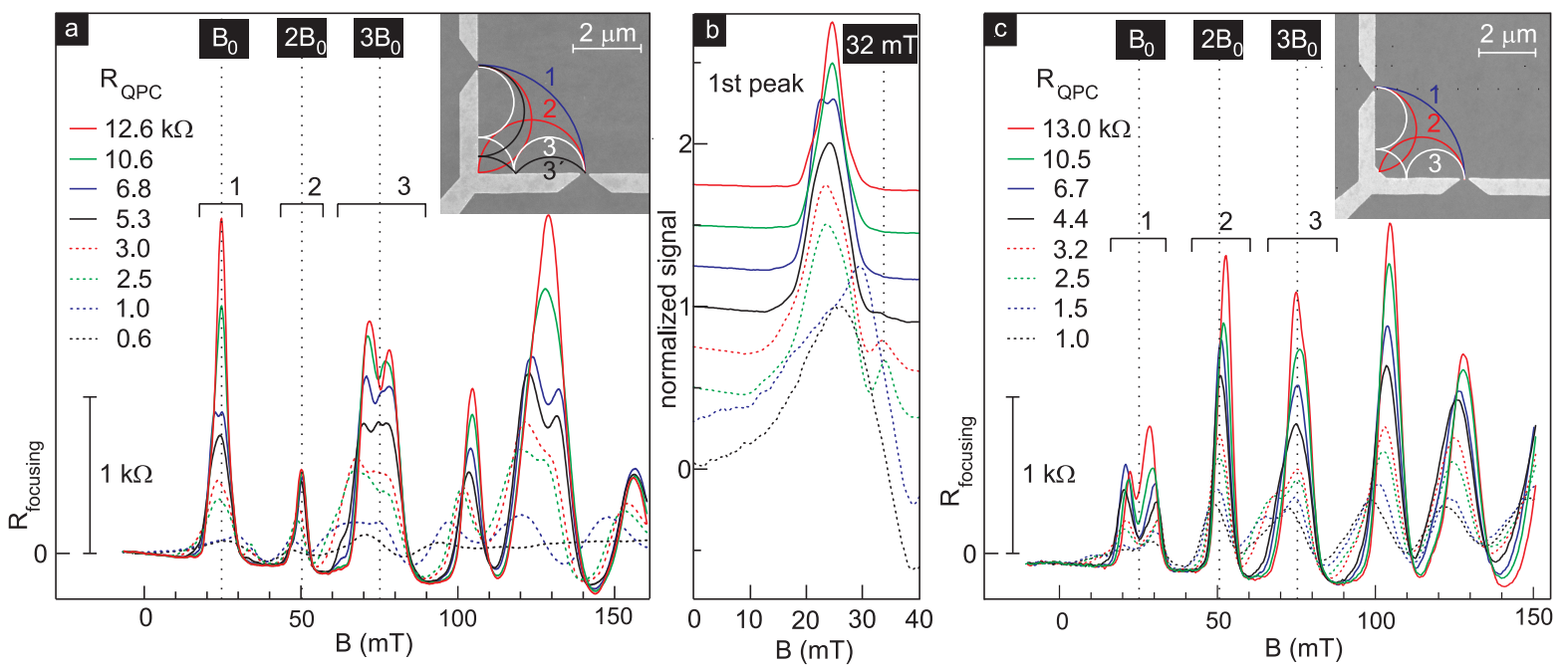

Figure 4.2: Experimental data. (a) Resistance curves for different magnetic fields show peaks at multiples of $B_{0}$ in a type I geometry. The peaks are very distinct, and clearly exhibit splitting, here in the third peak. Curves in different colors are for different values of the resistance across the QPC. The inset shows a picture of the experimental setup, together with some important trajectories (see text for details). (b) Detailed view of the first peak from (a). A small peak at a value of approximately $\sqrt{2} B_{0}$ is observed for weak resistances across the QPC (see sec. 4.3 for details) (c) Experimental data for type II geometry. Here, the first peak splits up. The peaks at even multiples of $B_{0}$ are larger due to the chamfered corner.

electron density $n$ is $n=2.5 \times 10^{11} \mathrm{~cm}^{-2}$ and $n=2.2 \times 10^{11} \mathrm{~cm}^{-2}$ for the type I and type II devices, respectively. The wall length from each QPC to the corner is $a=3 \mu \mathrm{m}$. The 2DEG exhibits an electron mean free path of $45 \mu \mathrm{m}$, one order of magnitude larger than the ballistic electron trajectories relevant for these studies. Transport measurements in a perpendicular magnetic field are carried out at $1.4 K$. The value of $B_{0}$ is given by $B_{0}=\hbar \sqrt{2 \pi n} / e a$, where $e$ is the electron charge.

A part of the experimental data is shown in fig. 4.2. together with images of the experimental setup in the insets, for the curves obtained here. It is observed that the experiment reproduces very clean peaks at regular intervals, just as is to be expected from a magnetic focusing experiment. However, several peaks show interesting substructure such as double-peaks. In particular, the first peak, which is attributed to trajectory 1 in the inset, is seen to split into two in several experiments conducted on different pieces of the heterostructure. In order to understand the experimental resistance curves, we first analyze the geometry and its influence on the peak structure. 

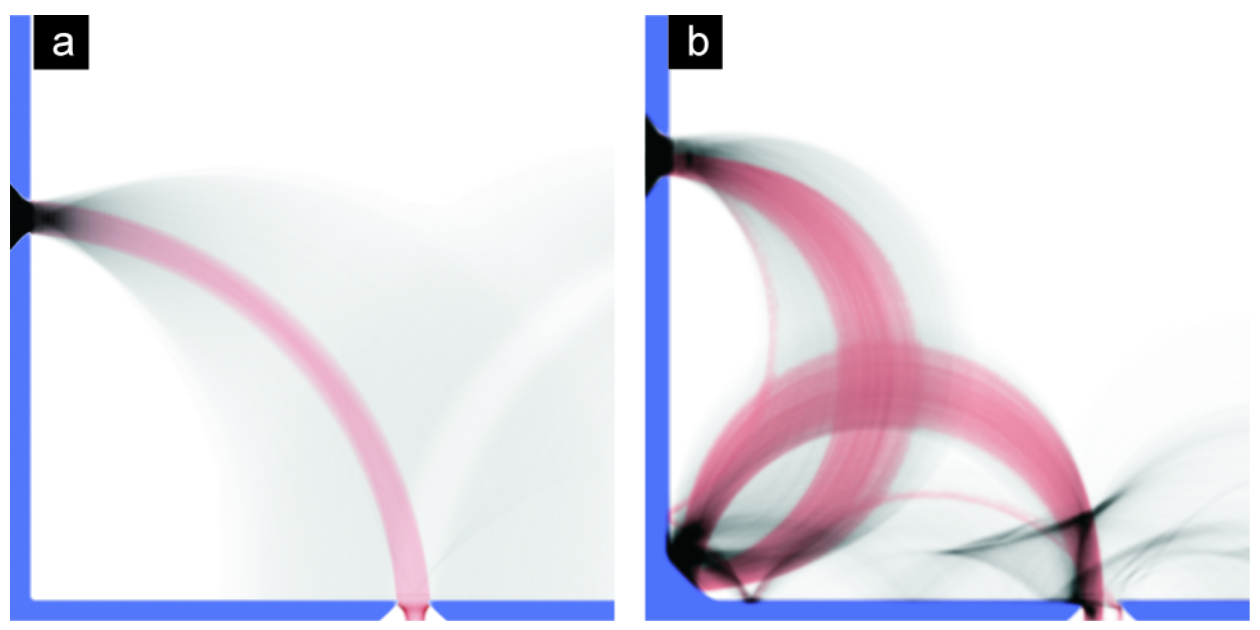

Figure 4.3: (a) Simulation of the particle flow (gray) in the corner devices of type I at $B=B_{0}$. Flow transmitted to the collector is additionally colored in red. The expected peak in the transmission at this value of $B$ is only due to the collimation of the beam by the $\mathrm{QPC}$, and not due to focusing. (b) Flow at $B=2 B_{0}$ in type II corner. The flow is focused both at the corner and at the receiver.

\subsection{Device geometry and its implications}

The corner device is different to the standard TMF experiment in several ways. Whereas in TMF, trajectories can be given explicitly in such a simple form that the caustics can be derived from it analytically [87], the trajectories in the corner geometry cannot be given in a simple way and the caustic structure is complicated. Most of our arguments therefore rely on detailed simulations of the corner device. Nevertheless, some important differences to TMF can be derived before studying the numerical results.

First of all, we note that the focusing occurs at different magnetic fields than naively expected. In particular, the peaks corresponding to the trajectories with odd numbers and therefore to odd multiples of $B_{0}$ in the insets of fig. 4.2 cannot be due to magnetic focusing because, as shown in the previous chapter, they focus after having traversed half a circle and not a quarter circle. Only the trajectories at even multiples of $B_{0}$ focus at the receiver, since these always hit the walls after traversing half a circle and can therefore be considered as being re-emitted from the wall as from a point contact (in the sense that a point source is nothing but a caustic, cf. chapter 3). Only for magnetic fields corresponding to the even trajectories should magnetic focusing be expected. What, however, are then the peaks corresponding to odd multiples of $B_{0}$ ? Let us look at two illustrations of the system for magnetic fields corresponding to trajectories 1 and 2, i.e. to magnetic fields $B_{0}$ and $2 B_{0}$.

A simulation of this case is shown in fig. 4.3, the gray scale indicates the particle density, while the flow that actually reaches the receiver is additionally colored in red. We observe that for $B=2 B_{0}$, (fig. $4.3 \mathrm{p}$ ), the transmitted flow is focused at the corner 


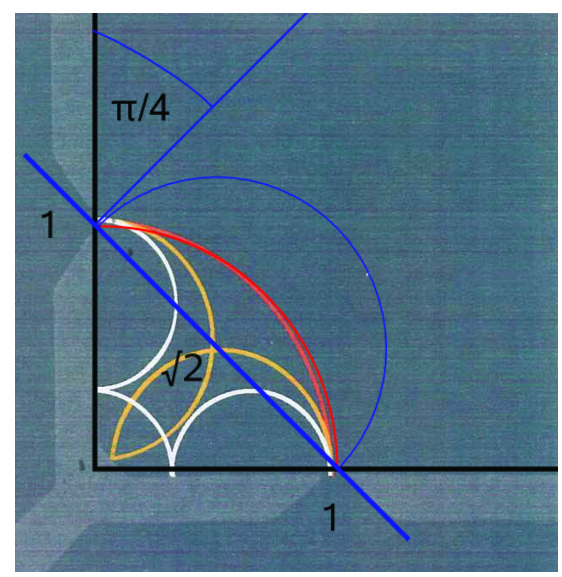

Figure 4.4: Illustration of the focusing geometry. Drawing an imaginary line between the QPCs, we observe that for values of $B=\sqrt{2} B_{0}$, corresponding to the blue trajectory (in comparison to the standard trajectories at integer multiples of $B_{0}$ ), the corner device acts like in the transverse magnetic focusing, although the caustic reaching the collector at this magnetic field is suppressed the more the beam is collimated.

and at the receiving QPC, as described above. One can clearly observe the focused beam to be diverging initially and then to recombine at the corner and the receiver, forming a caustic at these points. In contrast to this, the red flow in fig. 4.3 does not focus at the receiver. An increased density at this magnetic field can only be attributed to the collimation of the beam by the emitter, which causes most of the flow to have momentum mostly in the direction away from the QPC. These trajectories reach the collector at odd multiples of $B_{0}$ even though there is no focusing at this point. Because of this, we call peaks at odd multiples of $B_{0}$ collimation peaks, in contrast to the usual focusing peaks at even multiples of $B_{0}$. Before discussing the collimation of the QPC in sec. 4.3, we note several consequences of the above discussion. First, we show that between $B_{0}$ and $2 B_{0}$ there is a possibility for the electrons to focus as illustrated in fig. 4.4. If we draw an imaginary line from emitter to receiver, we see that we recover the TMF geometry, with the distance between the QPCs as $\sqrt{2} a$, and a corresponding focusing magnetic field of $\sqrt{2} B_{0}$, with the difference that electrons which focus at this magnetic field are emitted primarily in a direction tilted by $\pi / 4$ compared to the main perpendicular direction. The stronger the collimation of the emitter, the more this focusing peak can be suppressed. This explains why in the data we can see a small peak at $\sqrt{2} B_{0}$ (cf. fig. $4.2 \mathrm{~b}$ ) for small values of the resistance across the QPC, which determines the collimation of the QPC (see also sec. 4.3).

We also note a further difference to TMF. There exist orbits which are emitted perpendicularly but not received perpendicularly. They are also expected to show up in the transmission. We illustrate several orbits which are emitted perpendicularly in fig. 4.5. Calculating the magnetic fields at which the additional orbits are expected is a simple 


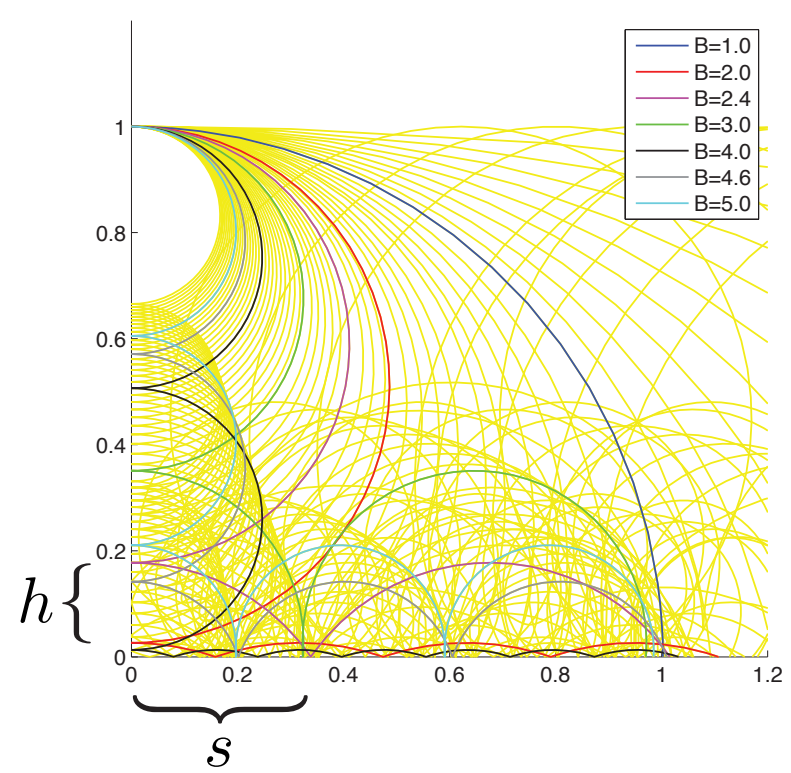

Figure 4.5: Perpendicularly emitted trajectories in a sharp-cornered device for different magnetic fields. The magnetic field is given in units of $B_{0}$ and the distance in units of $a$. The quantities $s$ and $h$ which are used to determine additional orbits are drawn for the trajectory at $B=2.4 B_{0}$. Orbits not colored in yellow are at multiples of $B_{0}$, and at the first two additional magnetic fields calculated in the text (approximately 2.4 and 4.6, see eq. (4.1)).

geometrical exercise: Assume the electrons to reflect specularly $p$ times at one wall, and to have a distance of $s$ to the corner at the last bounce. The distance from the corner to the first reflection point at the second wall is termed $h$ (see fig. 4.5), and therefore $h=1-2 p r$ with $r$ the cyclotron radius. Also,

$$
s=\sqrt{r^{2}-(r-h)^{2}}=\sqrt{r^{2}-(r+2 r p)^{2}}
$$

and we want $s=1 /(2 p+1)$ in order to find a trajectory which is emitted perpendicularly. Solving this for $r$ gives

$$
r=\frac{1+2 p+2 p^{2}}{2 p+6 p^{2}+4 p^{3}}
$$

and so the first two additional orbits are

$$
\begin{array}{cc}
B=12 / 5 B_{0}, & p=1 \\
B=60 / 13 B_{0}, & p=2 .
\end{array}
$$



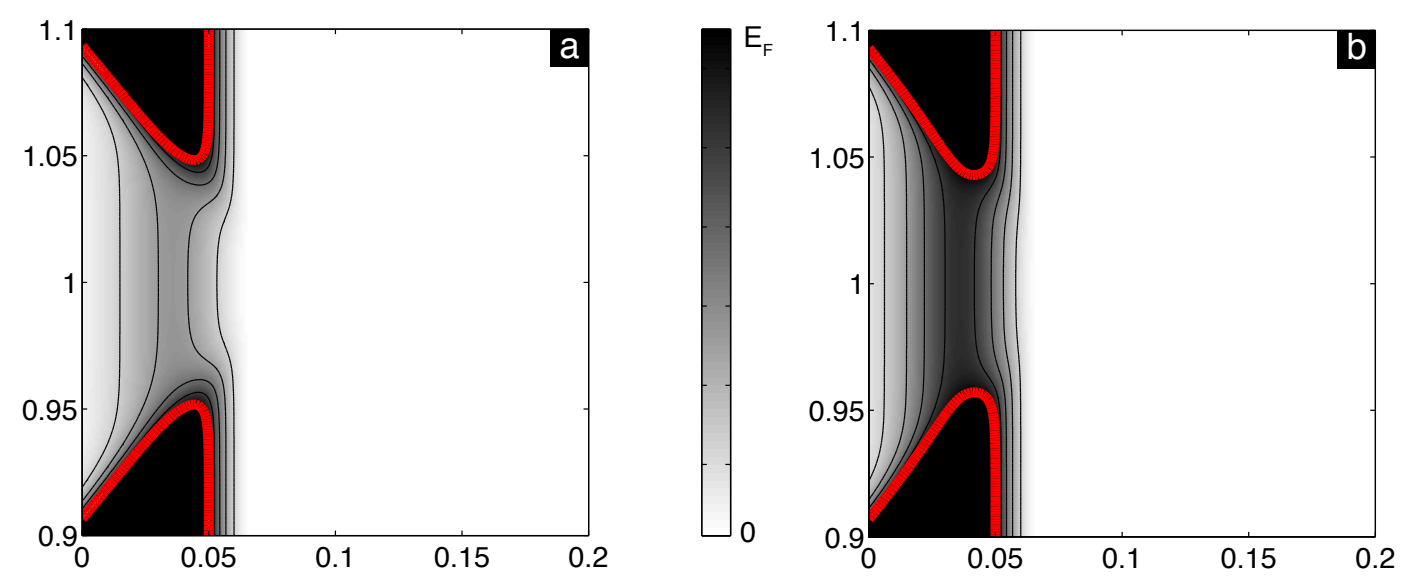

Figure 4.6: Saddle potential in the QPC. Parameters as given in appendix D, and $g_{0}=0.3$ for (a) and $g_{0}=0.6$ for (b), which corresponds to approximately 0.4 and 0.8 of the particles' energy, $E_{F}$. The red contour line denotes the value of $E_{F}$.

\subsection{Simulation of the system with quantum point contacts and soft walls}

The electrons move through the system almost freely, until they encounter the system boundaries, which consists of electrostatic walls from which the electrons reflect specularly. In an experiment, however, the electrostatic potential does not change abruptly from zero to a large value. Instead, the walls appear soft to the electrons. This is particularly important at the QPC: Because the walls are so close to each other at this point, we assume that the electrostatic potential does not drop to zero at the center of the opening, but that there is a saddle potential inside the QPC [89,95]. Including a saddle potential in the QPC also allows us to simulate different gate voltages at the QPC, which is used as an experimental parameter, and which can be used to control the collimation of the beam. We also assume that there is a lead attached to QPC from which electrons emerge with a cosine velocity distribution (see appendix C for details).

The precise definition of the model walls and QPCs is given in appendix D, together with the values of the parameters used for the simulations. We have checked numerically that our results do not depend strongly on the particular model we have chosen for the walls nor on the exact choice of parameters. We stress, however, the importance of the saddle since it allows us to simulate different collimation strengths of the QPC. Pictures of the QPC used in the simulations with two different saddle potential strengths are given in fig. 4.6. The potential energy is given as a percentage of the particles' energy, which in this chapter is denoted by $E_{F}$, the Fermi energy.

We can now examine the results of the transport simulations for different magnetic fields and for different saddle point potentials. The simulations are shown in fig. 4.7. All features discussed above manifest themselves in the simulations. A stronger saddle point 


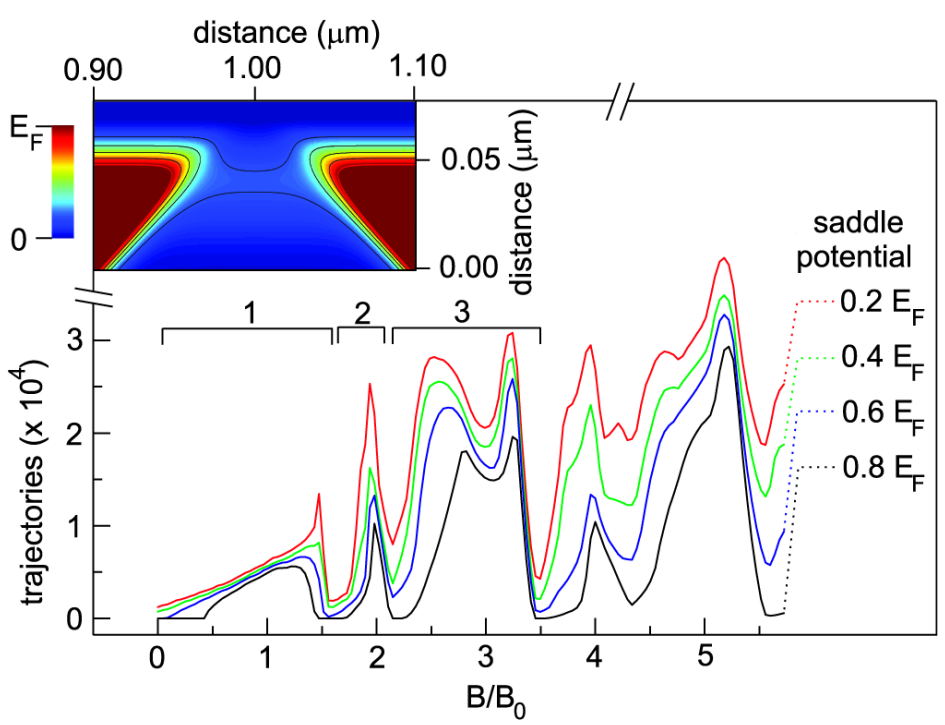

Figure 4.7: Simulated transmission curves for different saddle potentials (saddle illustrated in the inset). All features discussed in the previous sections, such as a caustic peak at $\sqrt{2} B_{0}$ which disappears for strong collimations of the beam and additional peaks at $2.4 B_{0}$ and $4.6 B_{0}$, can be observed.

potential causes a stronger collimation of the beam. This results in sharper peaks, as seen in the experiment. We also observe the predicted additional peaks at about $2.4 B_{0}$ and $4.6 B_{0}$, as well as the small caustic peak at $\sqrt{2} B_{0}$. As predicted, this peak is suppressed for stronger collimation, which means that the collimation peak at $B_{0}$ appears stronger. The collimation also, together with the soft walls, shifts the additional peak at $2.4 B_{0}$ towards the peak at $3 B_{0}$. We note that the focusing structure is complicated even in the idealized, clean case, and in particular depends strongly on the collimation of the beam. Since experimentally strong collimation peaks are observed, we will from now on assume a saddle potential of $0.8 E_{F}$.

\subsection{Quantum correction}

We briefly discuss the error we make in assuming classical propagation as opposed to quantum mechanical propagation when we deal with the location of caustics. This depends on the wavelength of the electrons which we consider, i.e. the Fermi wavelength associated with the Fermi energy. The Fermi energy is given by $\frac{\hbar^{2} k^{2}}{2 m}=\frac{n_{e}}{D O S_{2 D}}=n_{e} \frac{\pi \hbar^{2}}{m}$ where $n_{e}$ is the electron density, $k$ the Fermi wave vector, and $D O S_{2 D}$ is the density of states in two dimensions [96]. From the above calculation it follows that $k^{2}=2 \pi n_{e}$ and that the Fermi wavelength is given by

$$
\lambda_{F}=\frac{2 \pi}{k}=\sqrt{\frac{2 \pi}{n_{e}}} .
$$


In the experiment, $n_{e} \approx 2 \times 10^{11} \mathrm{~cm}^{-2}=2 \times 10^{15} \mathrm{~m}^{-2}$, and therefore $\lambda_{F} \approx 56 \mathrm{~nm}$. This is very small compared to the dimensions of the system, from which we conclude that quantum effects do not play an important role in the experiment. Additionally, we also check the expected quantum mechanical deviation of the first caustic from the classical calculation. The first caustic occurs, as shown in sec. 4.2, when trajectories have radius $r=a / \sqrt{2}$. The radius of the caustic is twice this radius, $\rho=2 r=\sqrt{2} a$. For a circular caustic, one can show that the highest intensity of the caustic is shifted quantum-mechanically from the classical position by [52]

$$
x_{\text {shift }}=1.02\left(\frac{\rho}{2 k^{2}}\right)^{1 / 3}=1.02\left(\frac{3 \sqrt{2}}{2 \times\left(1.12 \times 10^{2}\right)^{2}}\right)^{1 / 3} \approx 0.0564 \mu \mathrm{m}
$$

which is negligible and justifies our classical assumption.

\subsection{Adding a random potential}

A real 2DEG is never free from impurities. This is reflected in a finite mean free path (MFP) of the electrons. The experimentally measured value for the MFP is $\lambda_{m f p}=$ $45 \mu \mathrm{m}$, i.e. much larger than the system size $(a=3 \mu \mathrm{m})$. Experimentally, the MFP is determined by measuring the Hall resistance and the resistivity at zero magnetic field [97] of the unstructured sample, i.e. before the walls have been have been put in place. The main contribution to the measured MFP are the impurities of the semiconductor, which strongly scatter the electrons, and other bulk properties of the sample such as charged crystal defects and quantum corrections. The MFP is therefore also an approximate measure of the average distance between impurities. Since this is larger by an order of magnitude than the system size, there is normally no impurity in the region of system where transport takes place. We conclude from this that the real MFP inside the system is only a result of the small-angle scattering of the electrons by the disorder potentials created by the randomly distributed donor atoms, which create a random potential whose correlation function is approximately Gaussian [45]. As our model disorder potential, we therefore use a Gaussian correlated random potential which has a MFP which is one order of magnitude larger than the experimental one. The statistics of the random potential is not easily accessible experimentally. We therefore need to make realistic assumptions of the parameters. Specifically, we choose $\epsilon=2 \% E_{F}$ and $\ell_{c}=180 \mathrm{~nm}$, however, our results do not depend on this particular choice.

Even though the MFP is much greater than the system size, we see branching in the simulations on scales comparable to the system size. This is confirmed by the results derived in the previous chapter, where we calculated mean times to the first caustics in a random potential with a magnetic field. We plot again our scaling results from the previous chapter, this time with the parameters used for the simulations indicated (fig. 4.8 . 


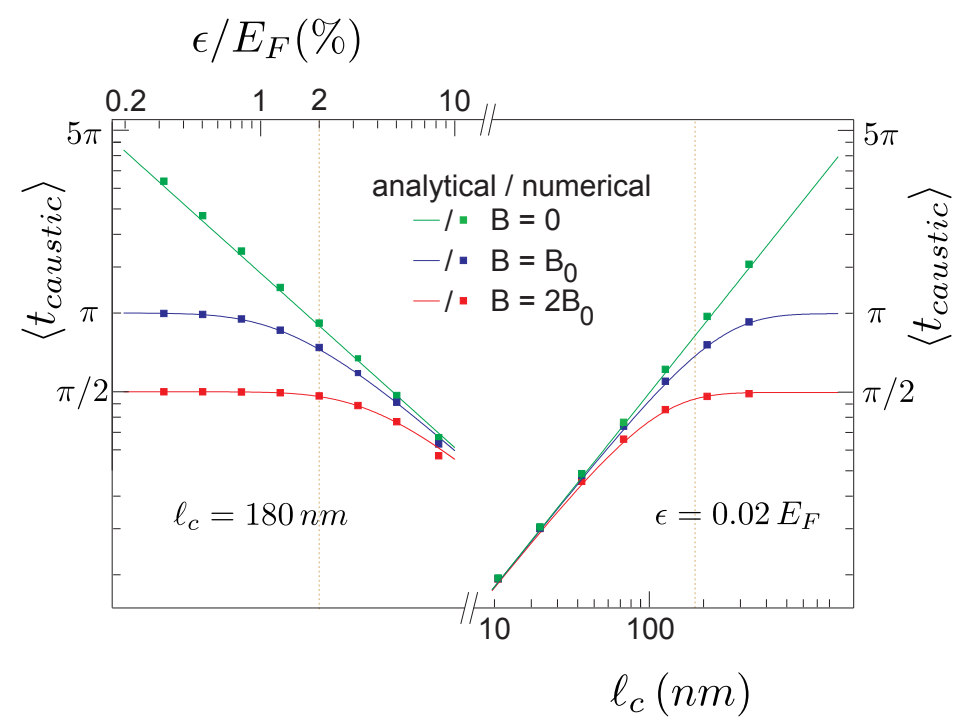

Figure 4.8: Scaling of the first caustic with the parameters of the random potential for different magnetic fields, derived in chapter 3. Here, we indicate with dotted vertical lines the parameters used for the simulation of the experiment, $\epsilon=2 \% E_{F}$ and $\ell_{c}=180 \mathrm{~nm}$. It can be observed that for these parameters, the random potential already has the effect of reducing the time to the first caustic from the expected case with only the magnetic field.

We observe that for these parameters, the random potential already has the effect of reducing the time to the first caustic from the expected case with only the magnetic field. We therefore expect branching to have a significant effect on the transport properties of our focusing device. This is most easily seen by adding a random potential to the simulations depicted in fig. 4.3 , which is shown in fig. 4.9 , with the plots from fig. 4.3 as insets.

We also find that the branching can have severe effects on the collimation peaks, enhancing, suppressing or altering them in other ways, while the focusing peaks are more robust to the disorder. This can be seen by looking at the two cases in fig. 4.9. Our interpretation is the following: The trajectories contributing to the collimation peak form a narrow bundle in phase space. If branching occurs, it is likely to influence the whole trajectory bundle. On the other hand, the trajectory bundle that is focused at a focusing peak has a broader structure in phase space (in position space this can be seen by the trajectory bundle opening between the QPCs and the walls), therefore there will always be some trajectories which focus at the collector, and the disorder only has a small effect on the focusing peak. Our analysis is confirmed by looking at several realizations of the random potential and the focusing curves that result from it, which is shown in fig. 4.10. We note that, depending on our realization, we can enhance, suppress or split the collimation peaks at odd multiples of $B_{0}$. The focusing peaks at even multiples are more robust to the disorder. We compare some of the simulated curves to experimental 

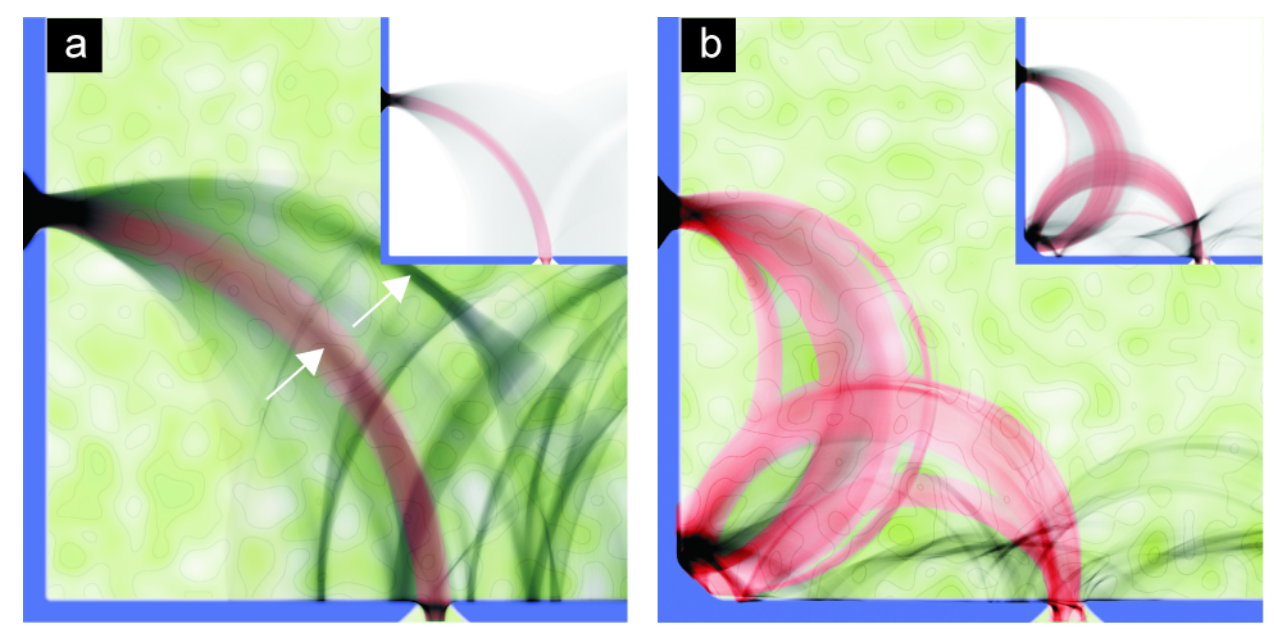

Figure 4.9: Simulations depicted in fig. 4.3 with an added random potential with parameters $\epsilon=2 \% E_{F}$ and $\ell_{c}=180 \mathrm{~nm}$. Insets show the original plots from fig. 4.3 (without random potential) as insets. (a) Caustics (indicated by arrows) form, here enhancing the collimation peak at $B_{0}$. (b) Although the random potential causes focusing, the magnetic focusing at $2 B_{0}$ is more robust to the effect of the random potential.

results in the next section.

\subsection{Comparison of experimental and simulated data}

We have shown that a random disorder, even though it is very weak, can strongly influence the transmission properties of the corner device. Since this is a statistical effect, it would be natural to try to obtain statistical quantities and compare these. However, the ensemble size is limited strongly in the experiment (it was performed with about ten different samples). We can therefore only compare realizations and show that we can qualitatively reproduce the experimental results. There are several features which are consistently reproduced in the simulations as well as the data: Most importantly, there is a probability for the collimation peaks at odd multiples of $B_{0}$ to split or show other substructure, while this effect is absent for the focusing peaks at even multiples of $B_{0}$. This effect can only be attributed to the random potential. Additional features such as the peak at $\sqrt{2} B_{0}$ and the additional focusing orbits are also observed in the experiment and the simulation, and can be enhanced or suppressed by the random potential. As an example, we compare two curves from fig. 4.2 with results from simulations in fig. 4.11 .

We additionally check that our results are not a consequence of the potential having a strong influence on the particles when they are slowing down at the QPC saddle potential by cutting out the random potential in this region. The result is shown in fig. 4.12. It shows that the random potential around the QPC is not important for the resulting transmission curve, but rather that the effects on the peak structure are due 


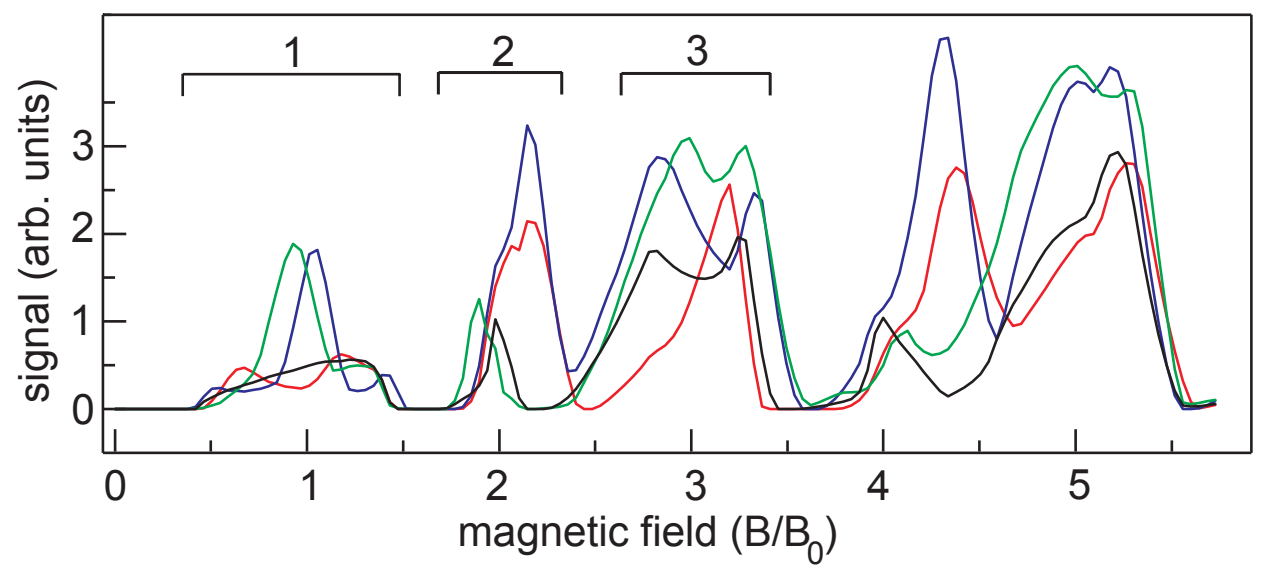

Figure 4.10: Simulated transmission curves for four different realizations of the random potential, blue and red curves obtained in a geometry with a chamfered corner and therefore with enhanced even peaks. The even peaks are robust against the disorder, while the odd peaks are strongly influenced by branching.
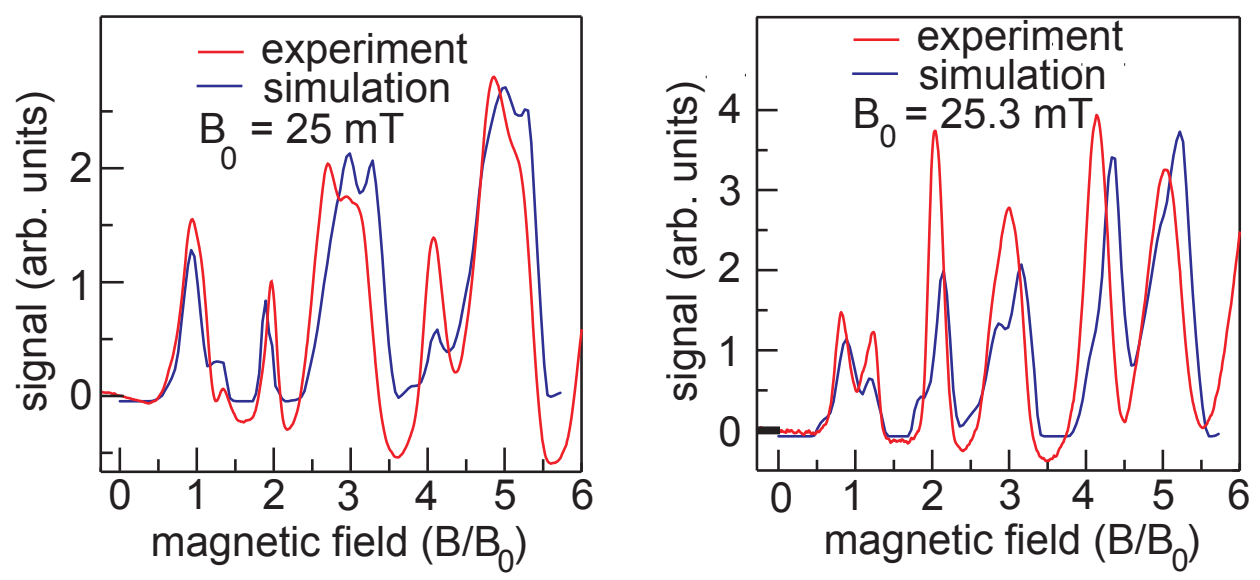

Figure 4.11: Comparison of experimental and simulated resistance curves for two experiments and two realizations of the random potential. The features of the experiment can qualitatively be reproduced. 


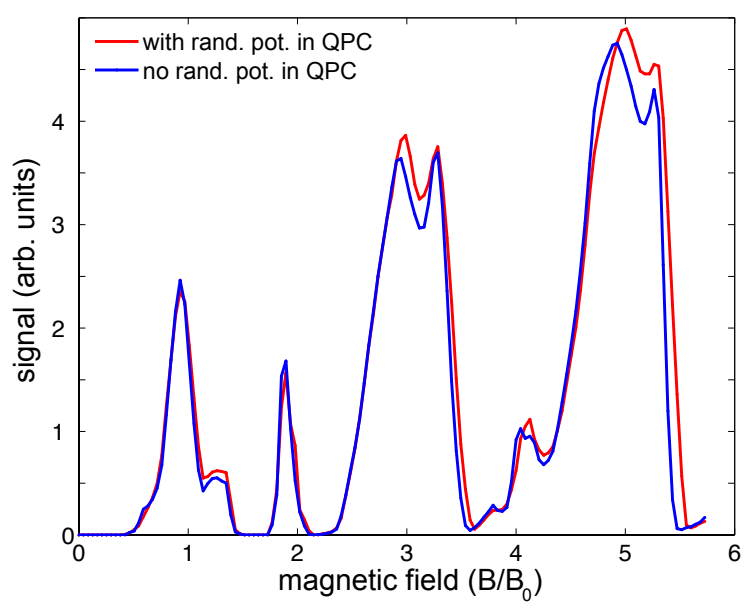

Figure 4.12: Comparison of a transmission curve with and without the random potential removed around the QPCs. The difference between the curves is negligibly small.

to the collective scattering of the potential, which is characteristic of branching.

\subsection{Conclusion}

In this chapter, we have applied methods and results derived in the previous chapter to a magnetic focusing experiment. A simulation of the geometry, including soft walls and a detailed model of the quantum point contact, already reveals several details seen in the experimental data. For a full understanding of the different appearances of the odd order peaks one needs to understand the difference between collimation and focusing peaks and, crucially, include a random potential in the simulations. This potential, although very weak, causes branching on scales comparable to the system size, which in turn can strongly influence the collimation peaks. All details of the experimental transmission curves have thus been reproduced in the simulations. Our findings show that a very weak random potential can strongly affect the DC conductance properties of very clean semiconductor microdevices, which has to be taken into account in the planning and fabrication of such devices. 



\section{Measures of stability and their relation to fold and cusp caustics}

We have seen that the caustic statistics calculated in chapter 3 have provided useful information about when to expect branching to occur in a random potential and a magnetic field, and we have used that knowledge in explaining experimental data in the previous chapter. Ultimately, however, we would like to move away from the Lagrangian description of the previous chapters to predictions which are more accessible to experiments, such as the density of caustics transverse to the direction of the flow. This will be achieved in the final chapter, however, some preliminary work is required. In particular, we will need to treat cusp caustics in more detail, since they are at the origin of every branch. As cusps are second-order singularities of the flow, we will need more information than is available in the curvature equation of chapter 3 . We therefore begin this chapter with a detailed account of the monodromy matrix, which takes into consideration the linearized surroundings of a trajectory, in order to then introduce an extended stability tensor, which will allow us to identify the locations of cusps. This will also be the basis for our numerical simulations in 2D. For analytical results, we again use the quasi-2D approximation. We derive equations for the entries of the monodromy matrix and the extended stability tensor in quasi-2D and study moments of their distribution analytically and numerically. In doing so, we take a closer look at the approximations needed for a quasi-2D treatment in terms of Fokker-Planck equations, as already mentioned in chapter 3 . In addition to this, we show how to reconcile the approach of finding caustics using the monodromy matrix with the approach using a curvature equation, as described in chapter 3.

The monodromy or stability matrix $\mathbf{M}$ plays an important role in the theory of dynamical systems and the study of chaos and quantum chaos. It describes the linearized phase space neighbourhood of a trajectory and is usually introduced when dealing with closed systems (e.g. $[65,98,99])$ where it is used to classify the stability of periodic orbits. It is also linked to the density of a flow, and therefore elements of it appear in the semiclassical (or Van Vleck) propagator [68]. Several other properties of the stability matrix are important in the study of dynamical systems, such as, most prominently, their eigenvalues (whose logarithms are the Lyapunov exponents in the limit of time going to infinity [100]). The definition of the stability matrix, however, is not limited to closed systems. Examples of successful applications to open systems include [41,42].

Caustics consist of points where trajectories which are initially separated by a small 
phase space distance coalesce in position space. As was shown in chapter 2 , the flow density diverges at these points, and the monodromy matrix can be used to locate them. We begin this chapter by showing how to perform this calculation for different initial conditions (the plane wave and point source initial conditions in 2D).

\subsection{Time evolution and initial conditions of the stability matrix}

In general, the monodromy matrix $\mathbf{M}$ with components $m_{i j}$ maps an initial phase space vector $\delta \mathbf{x}(0)$ with components $\delta x_{i}(0)$ onto a later one at time $t, \delta \mathbf{x}(t)$, such that

$$
\delta x_{i}(0)=\sum_{j} m_{i j} \delta x_{j}(t)
$$

The elements of $\mathbf{M}, m_{i j}$ are therefore given by

$$
m_{i j}(t)=\partial x_{i}(t) / \partial x_{j}(0)
$$

Clearly, $\mathbf{M}(0)=\mathbb{1}$ for all initial conditions of the Lagrangian manifold. The time evolution of $\mathbf{M}(t)$ is given by $[64,65,101]$

$$
\dot{\mathbf{M}}(t)=\mathbf{K}(t) \mathbf{M}(t)=\left(\begin{array}{cc}
0 & \mathbb{1} \\
-\mathbb{1} & 0
\end{array}\right)\left(\frac{\partial^{2} H}{\partial x_{i} \partial x_{j}}\right) \mathbf{M}(t)
$$

where $H$ is again the Hamiltonian. In case of Hamiltonians with terms including multiplication of position and momenta coordinates, the expression for $\mathbf{K}$ changes, but the expression here is sufficient for our Hamiltonian (eq. (2.2)).

\subsubsection{Initial conditions}

We now want to find the location of caustics using the results from chapter 2 where we derived the caustic condition

$$
\vec{p} \times \mathbf{P}_{\text {pos. }} \text { space }\left(\mathbf{M} d_{0}\right)=\left(-p_{y}, p_{x}, 0,0\right)^{T} \mathbf{M} \overrightarrow{d_{0}}=0 .
$$

With the time evolution of the monodromy given by eq. (5.1), we still require an expression for the initial displacement in phase space, $\vec{d}_{0}$.

We again consider our two standard initial conditions in $2 \mathrm{D}$, the plane wave and the point source. By fixing the total energy $E$ of the trajectories, conditions on all initial phase space displacements $\delta x_{i}^{0}=\delta x_{i}(0)$ can be derived. 


\subsubsection{Plane Wave}

The definition for the plane wave initial condition is $\delta p_{y}=0, \delta x=0$, and together with the initial condition for the constant energy shell $H=E$ this yields the desired conditions in the following way:

$$
\begin{gathered}
\frac{1}{2}\left(p_{x}^{0}+\delta p_{x}^{0}\right)^{2}+\frac{1}{2}\left(p_{y}^{0}\right)^{2}+V\left(x^{0}, y^{0}+\delta y^{0}\right) \\
\approx \frac{1}{2}\left(p_{x}^{0}\right)^{2}+\frac{1}{2}\left(p_{y}^{0}\right)^{2}+p_{x}^{0} \delta p_{x}^{0}+V\left(x^{0}, y^{0}\right)+V_{y}\left(x^{0}, y^{0}\right) \delta y^{0}=\frac{1}{2}\left(p_{x}^{0}\right)^{2}+\frac{1}{2}\left(p_{y}^{0}\right)^{2}+V\left(x^{0}, y^{0}\right) .
\end{gathered}
$$

Therefore, the dependence of $\delta p_{x}^{0}$ on $\delta y^{0}$ is given by

$$
\delta p_{x}^{0}=-\frac{V_{y}\left(x^{0}, y^{0}\right)}{p_{x}^{0}} \delta y^{0} \equiv \alpha \delta y^{0} .
$$

Acting on the initial displacement vector $\vec{d}_{0}$, this becomes

$$
\mathbf{M} \vec{d}_{0}=\mathbf{M}\left(\begin{array}{c}
\delta x^{0} \\
\delta y^{0} \\
\delta p_{x}^{0} \\
\delta p_{y}^{0}
\end{array}\right)=\mathbf{M}\left(\begin{array}{c}
0 \\
\delta y^{0} \\
\alpha \delta y^{0} \\
0
\end{array}\right)=\delta y^{0}\left(\begin{array}{c}
m_{12}+m_{13} \alpha \\
m_{22}+m_{23} \alpha \\
m_{32}+m_{33} \alpha \\
m_{42}+m_{43} \alpha
\end{array}\right)
$$

From eq. 5.2 , this gives the caustic condition

$$
\left(-p_{y}, p_{x}, 0,0\right)^{T} \mathbf{M} \overrightarrow{d_{0}}=-p_{y}\left(m_{12}+m_{13} \alpha\right)+p_{x}\left(m_{22}+m_{23} \alpha\right)=0 .
$$

\subsubsection{Point Source}

For the point source, the derivation is analogous, but this time with conditions $\delta x=$ $0, \delta y=0$, together with $H=E$. Therefore,

$$
\begin{gathered}
\left(p_{x}^{0}+\delta p_{x}^{0}\right)^{2}+\left(p_{y}^{0}+\delta p_{y}^{0}\right)^{2} \approx\left(p_{x}^{0}\right)^{2}+\left(p_{y}^{0}\right)^{0}+2\left(p_{x}^{0} \delta p_{x}^{0}+p_{y}^{0} \delta p_{y}^{0}\right) \\
\Rightarrow \delta p_{x}^{0}=-\frac{p_{y}^{0}}{p_{x}^{0}} \delta p_{y}^{0} \equiv \beta \delta p_{y}^{0} \\
M \vec{d}_{0}=M\left(\begin{array}{c}
\delta x^{0} \\
\delta y^{0} \\
\delta p_{x}^{0} \\
\delta p_{y}^{0}
\end{array}\right)=M\left(\begin{array}{c}
0 \\
0 \\
\beta \delta p_{y}^{0} \\
\delta p_{y}^{0}
\end{array}\right)=\delta p_{y}^{0}\left(\begin{array}{c}
m_{13} \beta+m_{14} \\
m_{23} \beta+m_{24} \\
m_{33} \beta+m_{34} \\
m_{43} \beta+m_{44}
\end{array}\right)
\end{gathered}
$$

and the caustic condition is

$$
\left(-p_{y}, p_{x}, 0,0\right)^{T} \mathbf{M} \overrightarrow{d_{0}}=-p_{y}\left(m_{13} \beta+m_{14}\right)+p_{x}\left(m_{23} \beta+m_{24}\right)=0 .
$$




\subsection{Cusps and the extended stability tensor}

Cusps are singular points of second order. This means that at a cusp, two lower order singularities (the folds) meet. Whereas at a fold, two trajectories coalesce, at a cusps, three trajectories coalesce. Inside the cusp, three initial conditions are mapped to one point in coordinate space, which causes an increased density in the cusp bounded by two folds. This is the reason why a branch does not only carry a lot of intensity at its borders, but there is also a density increase inside, as was shown in sec. 2.1.3.

For the plane wave initial condition in quasi-2D, the condition for a cusp is easily understood. It occurs at points where not only the first derivative $\partial y / \partial y_{0}$ is zero, but where the second one vanishes as well, i.e. $\partial^{2} y / \partial y_{0}^{2}=0$. This is the point where the Lagrangian manifold folds over and two turning-points are generated. These turning points coalesce initially, forming a singularity of second order which is the cusp.

For the multi-dimensional case, the situation is slightly more complicated and will be analyzed below. First, we introduce a generalization of the quasi-2D cusp condition, the extended stability tensor $\mathbf{N}$. The stability matrix provides information about the neighbourhood of the trajectory by linearizing the equations around the trajectory. The extended stability tensor takes into account the next order term, which is the higher derivative with respect to the initial conditions at $t=0$. Its components are defined by

$$
n_{i j k}:=\frac{\partial^{2} x_{i}(t)}{\partial x_{j}(0) \partial x_{k}(0)} .
$$

\subsubsection{Time evolution and initial conditions of the extended stability tensor}

The differential equations for the extended stability tensor are derived for the components as follows:

$$
\begin{aligned}
\dot{n}_{i j k} & =\frac{\partial}{\partial t}\left(\frac{\partial^{2} x_{i}(t)}{\partial x_{j}(0) \partial x_{k}(0)}\right)=\frac{\partial}{\partial x_{j}(0)}\left(\frac{\partial}{\partial t} \frac{\partial x_{i}(t)}{\partial x_{k}(0)}\right)=\frac{\partial}{\partial x_{j}(0)} \dot{m}_{i k} \\
& =\frac{\partial}{\partial x_{j}(0)} \sum_{b} \frac{\partial x_{b}(t)}{\partial x_{k}(0)} \frac{\partial \dot{x}_{i}(t)}{\partial x_{b}(t)} \\
& =\sum_{b}\left[\frac{\partial^{2} x_{b}(t)}{\partial x_{j}(0) \partial x_{k}(0)} \frac{\partial \dot{x}_{i}(t)}{\partial x_{b}(t)}+m_{b k} \frac{\partial}{\partial x_{j}(0)} \frac{\partial \dot{x}_{i}(t)}{\partial x_{b}(t)}\right] \\
& =\sum_{b}\left[n_{b j k} \frac{\partial \dot{x}_{i}(t)}{\partial x_{b}(t)}+m_{b k} \sum_{a} \frac{\partial x_{a}(t)}{\partial x_{j}(0)} \frac{\partial}{\partial x_{a}(t)} \frac{\partial \dot{x}_{i}(t)}{\partial x_{b}(t)}\right] \\
& =\sum_{b}\left[n_{b j k} \frac{\partial \dot{x}_{i}(t)}{\partial x_{b}(t)}+\sum_{a} m_{b k} m_{a j} \frac{\partial}{\partial x_{a}(t)} \frac{\partial \dot{x}_{i}(t)}{\partial x_{b}(t)}\right]
\end{aligned}
$$


which for two dimensions can be given as

$$
\dot{n}_{i j k}=\sum_{b} n_{b j k}\left(\begin{array}{cccc}
0 & 0 & 1 & 0 \\
0 & 0 & 0 & 1 \\
-V_{x x} & -V_{x y} & 0 & 0 \\
-V_{x y} & -V_{y y} & 0 & 0
\end{array}\right)_{i b}+\sum_{a, b} m_{a j} m_{b k} \frac{\partial}{\partial x_{a}(t)}\left(\begin{array}{cccc}
0 & 0 & 1 & 0 \\
0 & 0 & 0 & 1 \\
-V_{x x} & -V_{x y} & 0 & 0 \\
-V_{x y} & -V_{y y} & 0 & 0
\end{array}\right)_{i b}
$$

and the initial conditions for $n_{i j k}(t)$ are simply $n_{i j k}(0)=0$, because $\mathbf{N}$ is nothing but a derivative in several directions of the monodromy which is the unit matrix initially.

\subsubsection{Locating cusps using the extended stability tensor}

We have shown above how to obtain a condition for finding a first order caustic along a trajectory, involving the particles' momenta and entries of the monodromy matrix, i.e. the condition $\vec{p}_{\perp} \mathbf{M} \overrightarrow{d_{0}}=0$.

At a cusp, the first order caustic condition must be fulfilled, together with a higher order condition. It is crucial to note, however, that in more than one dimension, there is a non-trivial direction associated with every caustic and that the second order singularity condition has to be fulfilled with respect to this direction. This will become clear when looking at the $2 \mathrm{D}$ case, which we do next, and which can then be easily extended to higher dimensions.

In general, a trajectory defines a map from the initial conditions and the time traveled to the final position, e.g. for the plane wave initial condition in $2 \mathrm{D}$ this is denoted by $T:\left(y_{0}, t\right) \rightarrow(x, y)$. This is single valued initially, but soon becomes singular (noninvertible), when several initial condition are mapped to the same place ( $T$ goes from being bijective to surjective). The locations where the map is singular are just the caustics, and the map is singular when the Jacobian determinant $J$ vanishes. An explicit expression for $J$ is given by

$$
\begin{aligned}
J & =\operatorname{det}(D T)=\left|\begin{array}{cc}
\frac{\partial x}{\partial t} & \frac{\partial x}{\partial y_{0}} \\
\frac{\partial y}{\partial t} & \frac{\partial y}{\partial y_{0}}
\end{array}\right|=\frac{\partial x}{\partial t} \frac{\partial y}{\partial y_{0}}-\frac{\partial y}{\partial t} \frac{\partial x}{\partial y_{0}} \\
& =p_{x}\left(m_{22}+\frac{\partial y}{\partial p_{x}^{0}} \frac{\partial p_{x}^{0}}{\partial y_{0}}\right)-p_{y}\left(m_{12}+\frac{\partial x}{\partial p_{x}^{0}} \frac{\partial p_{x}^{0}}{\partial y_{0}}\right)=p_{x}\left(m_{22}+m_{23} \alpha\right)-p_{y}\left(m_{12}+m_{13} \alpha\right)
\end{aligned}
$$

which means that we have exactly recovered eq. (5.3). In addition, we can now identify the "direction" of the caustic. Since $T$ is singular at a caustic, it must have (at least) one vanishing eigenvalue. The eigenvector associated with this points in the direction we are looking for. To check whether we also are at a cusp, we must therefore take a derivative in the direction of the eigenvector $\vec{e}$ (with components $e_{1}$ and $e_{2}$ ) which is associated with the zero eigenvalue:

$$
\frac{\partial}{\partial \vec{e}} \operatorname{det}(D T)=\frac{\partial J}{\partial t} e_{1}+\frac{\partial J}{\partial y_{0}} e_{2}
$$




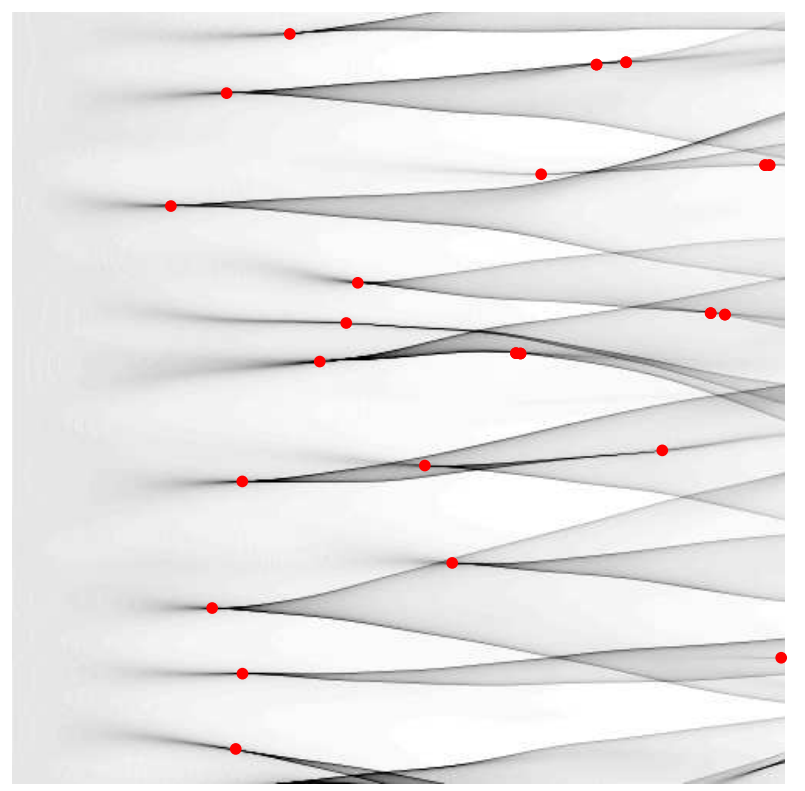

Figure 5.1: Two-dimensional simulation of a flow with plane wave initial condition. Cusp locations (red dots) are calculated using eq. (5.7).

A cusp is reached if both the caustic condition is fulfilled and (5.7) is zero. For completeness, we here give the expression for the direction of the eigenvector which is associated with the null eigenvalue for the plane wave (at least for relatively short times, where $x \approx t$, later, the other eigenvalue could be the one that vanishes):

$$
\vec{e}=\left(\left[+p_{x}-m_{22}-\sqrt{p_{x}^{2}+4 m_{12} p_{y}-2 p_{x} m_{22}+m_{22}^{2}}\right] / 2 p_{y}, 1\right)
$$

The other quantities in eq. (5.7) are

$$
\begin{aligned}
\frac{\partial J}{\partial t}= & -V_{x}\left(m_{22}+m_{23} \alpha\right)+p_{x}\left(m_{42}+m_{43} \alpha+m_{23} \alpha_{t}\right) \\
& +V_{y}\left(m_{12}+m_{13} \alpha\right)-p_{y}\left(m_{32}+m_{33} \alpha+m_{13} \alpha_{t}\right) \\
\frac{\partial J}{\partial y_{0}}= & p_{x}\left(n_{222}+n_{223} \alpha+m_{23} \alpha_{y_{0}}\right)+m_{32}\left(m_{22}+m_{23} \alpha\right) \\
& -p_{y}\left(n_{122}+n_{123} \alpha+m_{13} \alpha_{y_{0}}\right)-m_{42}\left(m_{12}+m_{13} \alpha\right)
\end{aligned}
$$

where now several elements of the stability tensor $n_{i j k}$ have been included. Fig. 5.1 shows the results of a simulation in which eq. (5.7) is used to determine the locations of the cusps. 


\subsection{Analytic results and the quasi-2D approximation}

In order to obtain analytical results for $\mathbf{M}$ and $\mathbf{N}$ in a random potential, we again reduce our system to the quasi-2D case, where time plays the role of the $x$-coordinate. We begin by rewriting the differential equations as first order stochastic differential equations, in order to allow a statistical treatment using Fokker-Planck equations later.

\subsubsection{Monodromy matrix in quasi-2D}

In quasi-2D, we are left with one spatial coordinate, $y$. The equations describing the mapping of an initial phase space displacement to a later time and the time evolution of the elements of the monodromy matrix derived from eq. (5.1) in $2 \mathrm{D}$ are given by

$$
\begin{aligned}
\left(\begin{array}{c}
\delta y \\
\delta p
\end{array}\right) & =\mathbf{M}\left(\begin{array}{c}
\delta y_{0} \\
\delta p_{0}
\end{array}\right)=\left(\begin{array}{ll}
\frac{\partial y_{t}}{\partial y_{0}} & \frac{\partial y_{t}}{\partial p_{0}} \\
\frac{\partial p_{t}}{\partial y_{0}} & \frac{\partial p_{t}}{\partial p_{0}}
\end{array}\right)\left(\begin{array}{c}
\delta y_{0} \\
\delta p_{0}
\end{array}\right) \\
\dot{\mathbf{M}} & =\mathbf{K} \mathbf{M}=\left(\begin{array}{cc}
0 & 1 \\
-\partial_{y y} V & 0
\end{array}\right)\left(\begin{array}{ll}
m_{11} & m_{12} \\
m_{21} & m_{22}
\end{array}\right)=\left(\begin{array}{cc}
m_{21} & m_{22} \\
-\partial_{y y} V m_{11} & -\partial_{y y} V m_{12}
\end{array}\right)
\end{aligned}
$$

and from the individual equations we can derive a second order equation for $m_{11}=$ $\partial y / \partial y_{0}$ :

$$
\begin{aligned}
\dot{m}_{11} & =m_{21} \\
\dot{m}_{21} & =-\partial_{y y} V m_{11} \\
\ddot{m}_{11} & =-\partial_{y y} V m_{11} \\
\ddot{m}_{11}(t)+\partial_{y y} V(t, y) m_{11}(t) & =0 .
\end{aligned}
$$

\subsubsection{Second-order stability tensor in quasi-2D}

The quasi-2D equivalent of eq. (5.5) is

$$
\begin{aligned}
\dot{n}_{i j k} & =\frac{\partial}{\partial t}\left(\frac{\partial^{2} x_{i}(t)}{\partial x_{j}(0) \partial x_{k}(0)}\right)=\frac{\partial}{\partial x_{j}(0)}\left(\frac{\partial}{\partial t} \frac{\partial x_{i}(t)}{\partial x_{k}(0)}\right)=\frac{\partial}{\partial x_{j}(0)} \dot{m}_{i k} \\
& =\left[\sum_{b} n_{b j k} \frac{\partial}{\partial x_{b}(t)}+\sum_{a, b} m_{a j} m_{b k} \frac{\partial^{2}}{\partial x_{a}(t) \partial x_{b}(t)}\right]\left(\frac{\partial x_{i}(t)}{\partial t}\right) \\
& {\left[\sum_{b} n_{b j k}+\sum_{a, b} m_{a j} m_{b k} \frac{\partial}{\partial x_{a}(t)}\right]\left(\begin{array}{cc}
0 & 1 \\
-\partial_{y y} V & 0
\end{array}\right)_{i b} }
\end{aligned}
$$


and the time evolution of $n_{111}$ is now given by

$$
\begin{aligned}
\dot{n}_{111} & =n_{211} \\
\dot{n}_{211} & =-n_{111} \partial_{y y} V-m_{11}^{2} \partial_{y y y} V \\
\Rightarrow \ddot{n}_{111} & =-n_{111} \partial_{y y} V-m_{11}^{2} \partial_{y y y} V .
\end{aligned}
$$

Note that we can also derive this directly from the equation for the first entry of monodromy matrix (5.8) as follows:

$$
\begin{array}{r}
\frac{\partial}{\partial y_{0}}\left[\ddot{m}_{11}(t)+\partial_{y y} V(t, y) m_{11}(t)\right]=0 \\
\ddot{n}_{111}+\partial_{y y} V(t, y) n_{111}+\partial_{y_{0}}\left(\partial_{y y} V(t, y)\right) m_{11}=0 \\
\ddot{n}_{111}+\partial_{y y} V(t, y) n_{111}+\left(\frac{\partial p}{\partial y_{0}} \partial_{p}+\frac{\partial y}{\partial y_{0}} \partial_{y}\right)\left(\partial_{y y} V(t, y)\right) m_{11}=0 \\
\ddot{n}_{111}+\partial_{y y} V(t, y) n_{111}+\frac{\partial p}{\partial y_{0}} \underbrace{\partial_{p}\left(\partial_{y y} V(t, y)\right)}_{=0} m_{11}+\partial_{y y y} V(t, y) m_{11}^{2}=0
\end{array}
$$

which is the same as eq. (5.9).

\subsubsection{Derivation of the Fokker-Planck equation}

We have seen that $\mathbf{N}$ depends on $\mathbf{M}$. Therefore, we will write a set of first-order coupled differential equations for the elements of the vector $\vec{a}(t)=(m(t), \dot{m}(t), n(t), \dot{n}(t))$, where we have shortened the notation $m \equiv m_{11}$ and $n \equiv n_{111}$ :

$$
\begin{aligned}
& \dot{a}_{1}(t)=a_{2}(t), \\
& \dot{a}_{2}(t)=-\partial_{y y} V(t, y) a_{1}(t), \\
& a_{1}(0)=1 \\
& \dot{a}_{3}(t)=a_{4}(t) \text {, } \\
& a_{2}(0)=0 \\
& \dot{a}_{4}(t)=-\partial_{y y} V(t, y) a_{3}(t)-\partial_{y y y} V(t, y) a_{1}^{2}(t), \\
& a_{3}(0)=0 \\
& a_{4}(0)=0 \text {. }
\end{aligned}
$$

The initial conditions have been included in accordance with sections 5.1 and 5.2 . In order to obtain a FPE, we must first assume again that the particles move fast in the $t$-direction (which is assumed to be equal to $x$ in the quasi-2D approach), and that the random potential appears to them as white noise in this direction. We write the equations 5.10 as

$$
\begin{array}{llrl}
\dot{a}_{1}(t) & =a_{2}(t), & & a_{1}(0)=1 \\
\dot{a}_{2}(t) & =\sigma_{1} \Gamma_{1}(t) a_{1}(t), & & a_{2}(0)=0 \\
\dot{a}_{3}(t) & =a_{4}(t), & & a_{3}(0)=0 \\
\dot{a}_{4}(t) & =\sigma_{1} \Gamma_{1}(t) a_{3}(t)+\sigma_{2} \Gamma_{2}(t) a_{1}^{2}(t), & & a_{4}(0)=0
\end{array}
$$


with

$$
\left\langle\Gamma_{i}(t) \Gamma_{j}\left(t^{\prime}\right)\right\rangle=2 \delta_{i j} \delta\left(t-t^{\prime}\right)
$$

The constants $\sigma_{1}$ and $\sigma_{2}$ are determined using the following argument: Although we assume delta-correlated noise in the $t$-direction, we want to retain the characteristics of the random potential in the transverse $y$-direction. This is achieved by keeping the integral over the correlation function constant, since this is related to the normalization of the potential, as discussed in [74,75,77,102-104]. We illustrate this in the general one-dimensional case first. Assume a correlation function $c\left(x-x^{\prime}\right)$ which we want to approximate as white noise,

$$
c\left(x-x^{\prime}\right) \rightarrow \delta\left(x-x^{\prime}\right)
$$

but which also conserves the integral of the correlation function. This is achieved by allowing

$$
c\left(x-x^{\prime}\right) \rightarrow \delta\left(x-x^{\prime}\right) \int_{-\infty}^{\infty} d x^{\prime \prime} c\left(x^{\prime \prime}\right)
$$

since then the integral of the right side of expression $(5.12)$ is then simply

$$
\int_{-\infty}^{\infty} d x \delta(x) \int_{-\infty}^{\infty} d x^{\prime \prime} c\left(x^{\prime \prime}\right)=\int_{-\infty}^{\infty} d x c(x)
$$

just as the integral of the left side of expression (5.12). For our random potential, this means that if we assume a transition to white noise (where we assume a Gaussian random field, allowing us to move the derivatives [61]) as

$$
\left\langle\partial_{y y} V(x, y) \partial_{y^{\prime} y^{\prime}} V\left(x^{\prime}, y^{\prime}\right)\right\rangle=\partial_{y y} \partial_{y^{\prime} y^{\prime}} c\left(x-x^{\prime}, y-y^{\prime}\right) \rightarrow \delta\left(x-x^{\prime}\right) 2 \sigma_{1}^{2}
$$

then the coefficient $\sigma_{1}$ in eq. (5.11) must now be given as an integral over the derivatives of the correlation function as

$$
\sigma_{1}^{2}=\left.\frac{1}{2} \int_{-\infty}^{\infty} d x \frac{\partial^{4} c(x, y)}{\partial y^{4}}\right|_{y=0} .
$$

This idea was already employed in chapter 3 , and we can now see that the $D$ defined in eq. (3.5) is related to $\sigma_{1}$ by $\sigma_{1}=D / \sqrt{2}$. In a similar way, the constant $\sigma_{2}$ can be shown to be

$$
\sigma_{2}^{2}=-\left.\frac{1}{2} \int_{-\infty}^{\infty} d x \frac{\partial^{6} c(x, y)}{\partial y^{6}}\right|_{y=0} .
$$

In order to give an expression for the FPE, we must now derive drift and diffusion coefficients for eqs. (5.11). By comparison with eqs. (B.6) from appendix B, we obtain 
for the drift coefficients

$$
\begin{aligned}
& D_{1}^{(1)}=a_{2} \\
& D_{3}^{(1)}=a_{4} \\
& D_{22}^{(2)}=\sigma_{1}^{2} a_{1}^{2} \\
& D_{24}^{(2)}=\sigma_{1} \sigma_{2} a_{1} a_{3} \\
& D_{42}^{(2)}=D_{24}^{(2)} \\
& D_{44}^{(2)}=\sigma_{1}^{2} a_{3}^{2}+\sigma_{2}^{2} a_{1}^{4}
\end{aligned}
$$

and all others are zero. Therefore, the FPE is given by

$$
\begin{aligned}
\partial_{t} P\left(\vec{a}, t \mid \vec{a}^{\prime}, t^{\prime}\right)= & {\left[-a_{2} \partial_{a_{1}}-a_{4} \partial_{a_{3}}+\sigma_{1}^{2} a_{1}^{2} \partial_{a_{2} a_{2}}+2 \sigma_{1}^{2} a_{1} a_{3} \partial_{a_{2} a_{4}}^{2}\right.} \\
& \left.+\partial_{a_{4} a_{4}}^{2}\left(\sigma_{2}^{2} a_{1}^{4}+\sigma_{1}^{2} a_{3}^{2}\right)\right] P\left(\vec{a}, t \mid \vec{a}^{\prime}, t^{\prime}\right) .
\end{aligned}
$$

We note that we use Stratonovich calculus, since this is a more natural choice when the white noise term is only an approximation of a correlation function with a certain width, as is the case here. Since the equations (5.11) are non-linear, so is eq. (5.13). It cannot be solved in general, so we turn to calculating moments of the elements of $\vec{a}(t)$.

\subsubsection{Moments of the distribution of the monodromy and the extended stability tensor}

In general, calculating moments from non-linear stochastic equations results in a recursive, non-closed set of ordinary differential equations for the moments. In our case, the equations are only weakly coupled (the equations for $m$ and $\dot{m}$ do not couple to $n$ and $\dot{n}$, but only the other way round), which is just enough to obtain closed equations for the moments. First, it is noted that all equations are symmetric around their average, and that therefore the odd moments are zero. We thus begin with the differential equations for the second moments of $m$ and $\dot{m}$ and denote by $k_{i}$ the second moments of the elements of $\vec{a}$ and their various combinations. Thus,

$$
\begin{aligned}
& \dot{k}_{1}=\left\langle\dot{a_{1}^{2}}\right\rangle=-\int d \vec{a} a_{1}^{2} a_{2} \partial_{1} P=2\left\langle a_{1} a_{2}\right\rangle=2 k_{2} \\
& \dot{k}_{2}=\left\langle a_{1} a_{2}\right\rangle=-\int d \vec{a} a_{2}^{2} a_{1} \partial_{1} P=\left\langle a_{2}^{2}\right\rangle=k_{3} \\
& \dot{k}_{3}=\left\langle\dot{a_{2}^{2}}\right\rangle=\sigma_{1}^{2} \int d \vec{a} a_{1}^{2} a_{2}^{2} \partial_{22} P=2 \sigma_{1}^{2}\left\langle a_{1}^{2}\right\rangle=2 \sigma_{1}^{2} k_{1}
\end{aligned}
$$

where the integrals are over all possible values from $-\infty$ to $\infty$ and are carried out using partial integration. We have obtain a closed set of equations which are linear in 
$\left\{k_{1}, k_{2}, k_{3}\right\}$ and which can easily be solved with initial conditions $k_{1}(0)=1, k_{2}(0)=$ $k_{3}(0)=0$. The result for the second moment of $m$ is

$$
k_{1}=\left\langle m^{2}(t)\right\rangle=\frac{1}{3}\left(\exp \left[\left(2 \sigma_{1}\right)^{2 / 3} t\right]+2 \exp \left[-\left(\sigma_{1} / \sqrt{2}\right)^{2 / 3} t\right] \cos \left(\sqrt{3}\left(\sigma_{1} / \sqrt{2}\right)^{2 / 3} t\right)\right.
$$

which is precisely the result obtained in [41], although obtained through a different method, as we describe at the end of this section. The equations for the second moments of $n$ and $\dot{n}$ are more complicated and involve the fourth moments of $m$. However, since this is in turn not coupled to $n$, again a closed set of equations can be obtained:

$$
\begin{aligned}
& \dot{k}_{4}=\left\langle\dot{a}_{3}^{2}\right\rangle=2\left\langle a_{3} a_{4}\right\rangle=2 k_{5} \\
& \dot{k}_{5}=\left\langle a_{3} a_{4}\right\rangle=\left\langle a_{4}^{2}\right\rangle=k_{6} \\
& \dot{k}_{6}=\left\langle\dot{a}_{4}^{2}\right\rangle=2 \sigma_{2}^{2}\left\langle a_{1}^{4}\right\rangle+2 \sigma_{1}^{2}\left\langle a_{3}^{2}\right\rangle=2 \sigma_{2}^{2} k_{7}+2 \sigma_{1}^{2} k_{4} \\
& \dot{k}_{7}=\left\langle\dot{a_{1}^{4}}\right\rangle=4\left\langle a_{1}^{3} a_{2}\right\rangle=4 k_{8} \\
& \dot{k}_{8}=\left\langle a_{1}^{3} a_{2}\right\rangle=3\left\langle a_{1}^{2} a_{2}^{2}\right\rangle=3 k_{9} \\
& \dot{k}_{9}=\left\langle a_{1}^{2} a_{2}^{2}\right\rangle=2\left\langle a_{1} a_{2}^{3}\right\rangle+2 \sigma_{1}^{2}\left\langle a_{1}^{4}\right\rangle=2 k_{10}+2 \sigma_{1}^{2} k_{7} \\
& \dot{k}_{10}=\left\langle a_{1} a_{2}^{3}\right\rangle=\left\langle a_{2}^{4}\right\rangle+6 \sigma_{1}^{2}\left\langle a_{1}^{3} a_{2}\right\rangle=k_{11}+6 \sigma_{1}^{2} k_{8} \\
& \dot{k}_{11}=\left\langle\dot{a}_{2}^{4}\right\rangle=12 \sigma_{1}^{2}\left\langle a_{1}^{2} a_{2}^{2}\right\rangle=12 \sigma_{1}^{2} k_{9} .
\end{aligned}
$$

The initial conditions for these $k_{i}$ are $k_{7}=1, k_{4}(0)=k_{5}(0)=k_{6}(0)=k_{8}(0)=k_{9}(0)=$ $k_{10}(0)=k_{11}(0)=0$. Again, the equations are closed and linear in $k_{i}$ and can be solved. Here, we give the result for the second moment of $n$, since this will be needed in the following chapter:

$$
\begin{aligned}
k_{4}=\left\langle n^{2}(t)\right\rangle & =\frac{\sigma_{2}^{2}}{210 \sigma_{1}^{2}}\left(98 e^{-\left(\sigma_{1} / \sqrt{2}\right)^{2 / 3} t} \cos \left(\sqrt{3}\left(\sigma_{1} / \sqrt{2}\right)^{2 / 3} t\right)+49 e^{\left(2 \sigma_{1}\right)^{2 / 3} t}+e^{21^{1 / 3}\left(2 \sigma_{1}\right)^{2 / 3} t}\right. \\
& \left.+2 e^{-(21 / 2)^{1 / 3} \sigma_{1}^{2 / 3} t} \cos \left(\left(73^{5 / 2} / 2\right)^{1 / 3} \sigma_{1}^{2 / 3} t\right)-150\right) .
\end{aligned}
$$

The analytical results are compared to numerical simulations in fig. 5.2. We obtain very good agreement with simulations, although the results are only exact in the limit $\epsilon \rightarrow 0$.

An example for the expressions for $\sigma_{1}$ and $\sigma_{2}$ for the Gaussian correlated random potential are given by

$$
\begin{aligned}
\sigma_{1}^{2} & =6 \sqrt{\pi} \epsilon^{2} \ell_{c}^{-3} \\
\sigma_{2}^{2} & =60 \sqrt{\pi} \epsilon^{2} \ell_{c}^{-5}=10 \sigma_{1}^{2} \ell_{c}^{-2} .
\end{aligned}
$$

We stress, however, that our results are valid for other correlation functions as well, as will be shown in the following chapter. In particular, not only the values for $\sigma_{1}$ and 


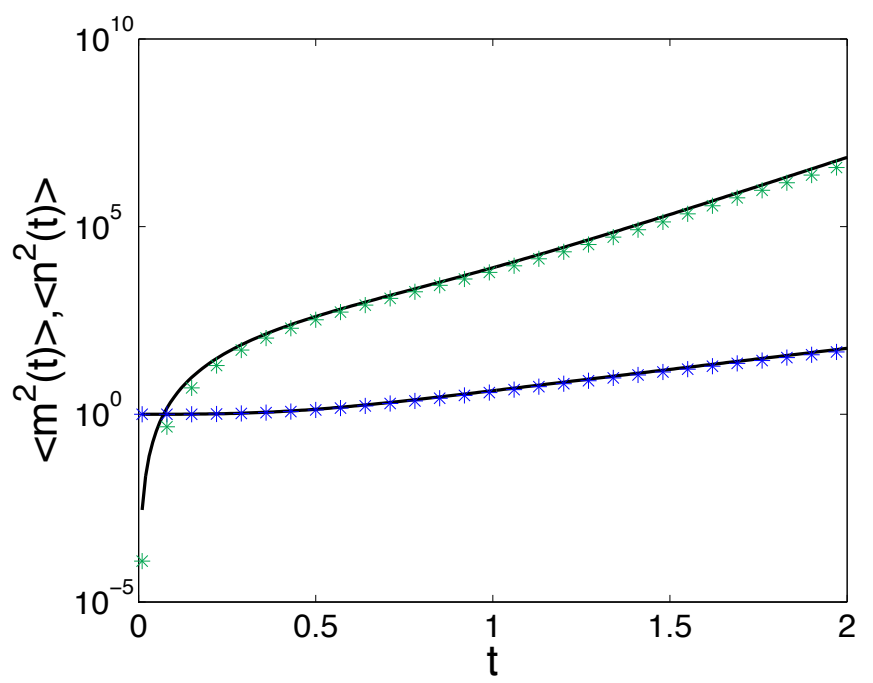

Figure 5.2: Second moments of $m$ and $n$ for $\epsilon=0.02, \ell_{c}=0.1$. Analytics (black) match well the numerical data (blue for $\left\langle m^{2}\right\rangle$, green for $\left\langle n^{2}\right\rangle$ ).

$\sigma_{2}$ differ for different correlation functions, but their ratio can also be different. For example, the ratio $\sigma_{2} / \sigma_{1}$ is $\sqrt{10} / \ell_{c}$ for the Gaussian correlation function and $\sqrt{45} / \ell_{c}$ for a power-law correlation function $c(r)=\left(1+r^{2} / \ell_{c}^{2}\right)^{-4}$. The ratio appears in the expression for the second moment of $n$, eq. (5.15), and will become important in the next chapter.

We also note that our analysis is not limited to the second moments. Indeed, for the calculation of the second moments of $n$ and $\dot{n}$, the fourth moments of $m$ have already been used, and similarly higher moments could be obtained for all quantities. Since we will only need second moments in the next chapter, we refrain from giving higher moments here.

Finally, we compare the result for the first entry of the monodromy matrix to the results in the literature [41,42]. There, for long times a scaling of $\left\langle a_{1}^{2}\right\rangle \sim \frac{1}{3} \exp \left(2 \nu^{\prime} t\right)$ was derived which, for the Gaussian correlated field, gives an exponent in front of $t$ of the form

$$
2 \nu^{\prime}=2(3 \sqrt{\pi})^{1 / 3} \epsilon^{2 / 3} / \ell_{c}
$$

When following our derivation, we obtain a long-term scaling of (cf. eq. (5.14))

$$
\left\langle a_{1}^{2}\right\rangle \sim \frac{1}{3} \exp \left(\left(2 \sigma_{1}\right)^{2 / 3} t\right)
$$

which, assuming a Gaussian correlation function $c(x, y)=\epsilon^{2} e^{-\left(x^{2}+y^{2}\right) / \ell_{c}^{2}}$, gives an exponent of

$$
\left(2 \sigma_{1}\right)^{2 / 3}=\left(24 \sqrt{\pi} \epsilon^{2} \ell_{c}^{-3}\right)^{1 / 3}=2(3 \sqrt{\pi})^{1 / 3} \epsilon^{2 / 3} / \ell_{c}
$$

which is exactly the same as eq. (5.16). 


\subsection{Connection to curvature equation}

We have given two seemingly unrelated approaches to obtaining an equation for the location of caustics. On the one hand, the curvature equation (cf. chap. 3), which is a first order, non-linear stochastic differential equation with additive noise (i.e. the random potential), and on the other hand, a second order linear stochastic differential equation with multiplicative noise for the first component of the monodromy matrix, $m_{11}$, as derived in this chapter. A relation between $m_{11}$ and the curvature $u$ can easily be derived in the quasi-2D case:

$$
u=\frac{\partial p}{\partial x}=\frac{\partial \dot{x}}{\partial x}=\frac{\partial x_{0}}{\partial x} \frac{\partial \dot{x}}{\partial x_{0}}=\frac{\dot{m}_{11}}{m_{11}}=\frac{m_{21}}{m_{11}}
$$

which implies that $u \rightarrow \infty$ is equivalent to $m_{11} \rightarrow 0$, which both signal the appearance of a caustic. This can also be shown in terms of the equations governing the evolution of $u$ and $m_{11}$ in the following way:

We start with the equation for $m_{11}$ (eq. (5.8)) and convert it into an equation with additive noise, taking into account that we assume the potential to be $\delta$-correlated in the time direction, i.e. a Wiener process $W$. We first change notation to the the usual differential notation for stochastic equations, $m_{11} \equiv x_{1}, \dot{m}_{11} \equiv x_{2}$ and we use $\sigma \equiv \sigma_{1}$ from sec. 5.3 .3 to obtain

$$
\begin{aligned}
& d x_{1}=x_{2} d t \\
& d x_{2}=\sigma x_{1} d W .
\end{aligned}
$$

We now seek a way to make the noise non-multiplicative. As opposed to the rest of the thesis, we here use Ito calculus, as the additional terms compared to Stratonovich calculus will be chosen to vanish anyway, which will make this result general for both choices. In general, if we have two functions $f_{1}\left(x_{1}, x_{2}, W\right), f_{2}\left(x_{1}, x_{2}, W\right)$ we get from Ito's Lemma [105]

$$
\begin{aligned}
d f_{1} & =\partial_{1} f_{1} d x_{1}+\partial_{2} f_{1} d x_{2}+\frac{1}{2} \partial_{11} f_{1} d x_{1}^{2}+\frac{1}{2} \partial_{22} f_{1} d x_{2}^{2}+\partial_{12} f_{1} d x_{1} d x_{2} \\
& =\partial_{1} f_{1} d x_{1}+\partial_{2} f_{1} d x_{2}+\mathcal{O}\left(d t^{2}\right)+\frac{1}{2} \partial_{22} f_{1} d x_{2}^{2}+\mathcal{O}(d t d W) \\
\Rightarrow d f_{1} & =\partial_{1} f_{1} x_{2} d t+\partial_{2} f_{1} \sigma x_{1} d W+\frac{1}{2} \partial_{22} f_{1} \sigma^{2} x_{1}^{2} d t \\
& =\left(\partial_{1} f_{1} x_{2}+\frac{1}{2} \partial_{22} f_{1} \sigma^{2} x_{1}^{2}\right) d t+\partial_{2} f_{1} \sigma x_{1} d W
\end{aligned}
$$

and similarly for $f_{2}$

$$
d f_{2}=\left(\partial_{1} f_{2} x_{2}+\frac{1}{2} \partial_{22} f_{2} \sigma^{2} x_{1}^{2}\right) d t+\partial_{2} f_{2} \sigma x_{1} d W .
$$

We now impose $\partial_{2} f_{2}=\frac{c}{x_{1}}$ and $\partial_{2} f_{1}=0$, since this will cause the term with $d W$ in $f_{1}$ to vanish and in $f_{2}$ to not contain a term in $x_{1}$ or $x_{2}$, i.e. it will make the noise additive. We 
therefore have $f_{1}=b\left(x_{1}\right)$ and $f_{2}=x_{2} \frac{c}{x_{1}}$ and we are now at the point where Stratonovich calculus would give the same results. Plugging $f_{1}$ and $f_{2}$ into (5.17) and (5.18) we obtain

$$
\begin{aligned}
& d f_{1}=b^{\prime}\left(x_{1}\right) x_{2} d t \\
& d f_{2}=\left(-c \frac{x_{2}}{x_{1}^{2}}\right) x_{2} d t+c \sigma d W
\end{aligned}
$$

where the prime denotes the derivative. So $f_{2}=c \frac{x_{2}}{x_{1}}$, and we also choose $f_{1}\left(x_{1}\right)=$ $b\left(x_{1}\right)=b_{1} x_{1}+b_{2}$. Then $x_{2}=f_{2} x_{1} / c=\frac{f_{2}\left(f_{1}-b_{2}\right)}{c b_{1}}$

$$
\begin{aligned}
& d f_{1}=b_{1} x_{2} d t=\frac{f_{2}\left(f_{1}-b_{2}\right)}{c} d t \\
& d f_{2}=-c \frac{x_{2}^{2}}{x_{1}^{2}} d t+c \sigma d W=-\frac{1}{c} f_{2}^{2} d t+c \sigma d W
\end{aligned}
$$

and we can put $c=1$. We have now achieved our aim: We have decoupled $f_{2}$ from $f_{1}$ and turned it into an equation with additive noise. The price to pay is that the equation is now non-linear. We note that when $f_{2} \rightarrow \infty, x_{1} \rightarrow 0$, which is just the condition for a caustic. This means that we have fully recovered the curvature equation if we interpret $f_{2}$ as $u$, i.e.

$$
d u=-u^{2} d t+\sigma d W
$$

which is eq. (3.3) in the form used by White et al. [72].

\subsection{Conclusion}

This chapter has been devoted to analysing the stability of trajectories in a random potential, and how this is related to caustics. We have given a detailed account of how to find caustics using the stability matrix for different initial conditions. We have also introduced a second-order stability tensor which gives information on higher-order stability properties of trajectories. The stability tensor appears naturally in the study of cusps, and therefore, implicitly, in the statistics of branches which will be the subject of the next chapter. The statistics of the elements of the stability matrix and the stability tensor have been studied analytically using a quasi-2D approach. Although the equations for the elements of the stability tensor are nonlinear, we have succeeded in calculating moments of their distribution. All results have been confirmed numerically using quasi-2D and fully 2D simulation (some of which are non-trivial, for example, for the 2D for the simulations of the extended stability tensor one needs to simultaneously solve 60 coupled differential equations). Finally, we have shown how to reconcile the approach of this chapter with the approach using the curvature equation from chapter 3. 


\section{Universal Branch Statistics}

In previous chapters, the theoretical effort was concentrated on Lagrangian statistics of caustics, stability matrix elements, and other quantities along a trajectory. This is also the approach taken in a large part of the literature. It is a very valuable method since it allows us to study ordinary stochastic differential equations instead of partial ones. Nevertheless, most of the quantities which we can observe are measured in a fixed coordinate system and not in the constantly changing one of the trajectories. In addition to this, for most systems we consider the classical trajectories to be only approximations to the actual wave-like systems.

It was demonstrated in previous chapters that in cases where we were able to identify the time taken along a trajectory with a spatial coordinate, we subsequently were able to draw some conclusions about observable quantities, for example, about the mean time and therefore the mean approximate distance to a caustic. This was possible because of the particles moving in this direction almost freely. However, once we venture into the direction transverse to the main direction of the flow, the motion is only governed by the random potential, and there is no Lagrangian quantity which can easily be attributed to an Eulerian (i.e. fixed-frame) quantity.

This chapter will be devoted to obtaining Eulerian caustic statistics, which arise naturally when one asks one of the most elementary questions that comes to mind when analyzing branched flow: How many caustics, on average, can be observed per unit transversal distance as a function of the distance from the source? The problem is illustrated in fig. 6.1, together with a cut through the flow density, which could be measured in an experiment and from which the number of branches can be extracted.

The question of how many caustics can be observed at a distance was answered for the much simpler case of initially randomly distorted trajectories, which then travel in straight lines through a constant potential by Berry and Upstill [20]. We briefly review this analysis and confirm it numerically. We then answer the question for a plane wave initial condition and an extended random medium. In doing so, we combine several results from the literature and results obtained in previous chapters to, in the end, arrive at a universal caustic distribution. As in the previous chapter, we also take into account different correlation functions. All of the analytical calculations will be done using the quasi-2D approach, but we will compare with full $2 \mathrm{D}$ numerics as well. The results are published in [106] 


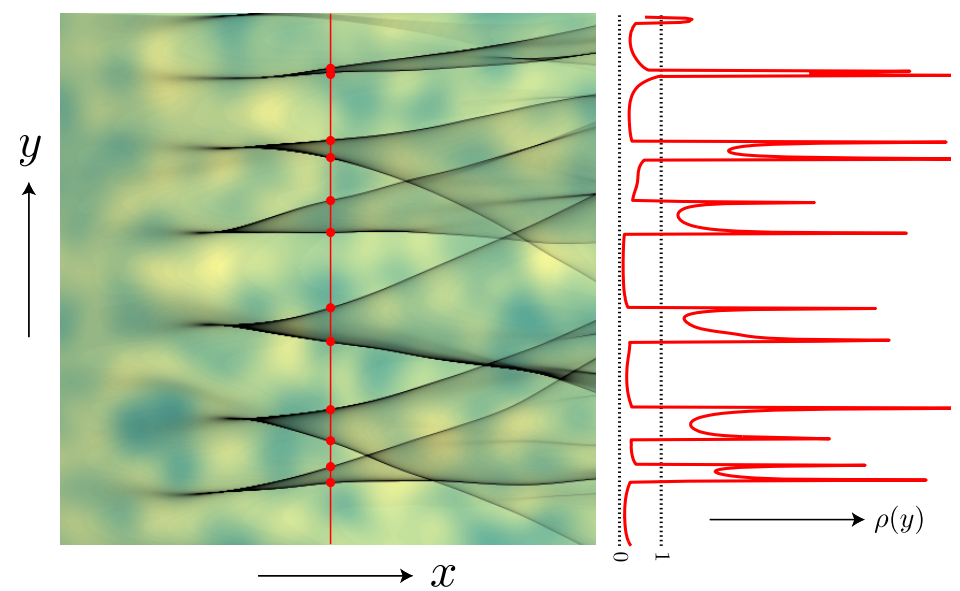

Figure 6.1: Flow density in a random potential. We want obtain the number of caustics (red dots) along the red line at some distance from the source perpendicular to the flow. To the right, the density across this line is shown. Branches show up clearly as bounded by two caustics, with increased density at the core, as was explained in chapter 2 .

\subsection{Counting caustics, counting branches}

First, we give a general expression which will allow us to calculate the number of caustics at distance $t$ per unit distance in the transverse direction. It is given by

$$
N_{\text {caustic }}(t)=\lim _{L \rightarrow \infty} \frac{1}{L}\left\langle\int_{0}^{L} d y_{0} \delta\left(\gamma\left(t, y_{0}\right)\right)\left|\frac{\partial \gamma\left(t, y_{0}\right)}{\partial y_{0}}\right|\right\rangle
$$

where $\gamma(t)$ is zero when a branch is encountered while integrating over $y_{0}$ and where the average is over realizations of the statistical quantities involved (either initial conditions as in sec. 6.2, or over random potentials as in sec. 6.4. We note that the Jacobian appearing in the expression ensures that every caustic is counted with the same weight, such that $N_{\text {caustic }}$ actually increases by one whenever a caustic is encountered along the integration path.

\subsection{Random initial condition without random potential}

Consider a slightly deformed initial plane wave, with $f(y)$ describing the deviation from the plane wave $f(y)=0$ and assuming that $f$ is random with a given correlation function. Then the number of fold lines (fold caustics) at time $t$ (or at a distance $x$ ) $N_{f}(t)$ per unit distance in the (transverse) $y$ direction is given by [20] 


$$
N_{f}(t)=\lim _{L \rightarrow \infty} \frac{1}{L}\left\langle\int_{0}^{L} d y_{0} \delta\left(1-t f_{y y}\right)\left|t f_{y y y}\right|\right\rangle
$$

where the quantity inside the delta function is just the condition for the caustic, and $\left|t f_{y y y}\right|$ is the Jacobian, as explained for eq. 6.1). Since one can show $f_{y y}$ and $f_{y y y}$ to be statistically independent, the equation simplifies to

$$
N_{f}(t)=\left\langle\delta\left(1-t f_{y y}\right)\right\rangle\left\langle\left|t f_{y y y}\right|\right\rangle .
$$

In chapter 3 we have calculated the probability to reach a caustic along a trajectory, $P_{c}(t)$. It will be useful to formulate the equation for $N_{f}(t)$ in terms of $P_{c}(t)$. We perform the calculation for this simple case for illustration purposes, since the same method will be used later for the problem including the random potential. Denoting $f_{y y}=u$ one obtains

$$
P_{c}(t)=\langle\delta(t-1 / u)\rangle=\int d u \delta(t-1 / u) P(u)=\sum_{i} \frac{P\left(u_{i}\right)}{\left|\frac{\partial(t-1 / u)}{\partial u}\right|_{u=1 / t} \mid}=\frac{P(1 / t)}{t^{2}}
$$

and

$$
\langle\delta(1-u t)\rangle=\int d u \delta(1-u t) P(u)=\int d u \frac{1}{t} \delta(1 / t-u) P(u)=\frac{P(1 / t)}{t}=P_{c}(t) t
$$

SO

$$
N_{f}(t)=P_{c} t\left\langle\left|t f_{y y y}\right|\right\rangle=\frac{F_{3}}{\pi F_{2}} \exp \left[\left(-2 t^{2} F_{2}^{2}\right)^{-1}\right]
$$

with $F_{i}=\left[\left\langle\left(\frac{d^{i} f}{d x^{i}}\right)^{2}\right\rangle\right]^{1 / 2}$ (see [20] for details).

We first turn to the numeric calculation of $N_{f}$ in order to confirm the results from [20] and then compare them to the case of a continuous random potential, which will be treated in sec. 6.4.

\subsection{Counting branches numerically}

In order to verify our analytical results, we will need accurate numerical simulations of the number of branches at a given distance. However, all our numerical methods developed in the previous chapters are Lagrangian methods and determine the statistics of caustics along a trajectory, but not the transverse caustic density. One possibility would be to use an Eulerian integration method [107-109], however, this amounts to solving the HJE in addition to an equation for the caustics and is computationally expensive. Therefore, we develop two simpler methods based on our Lagrangian caustic location code. 


\subsubsection{Direct trajectory method}

The first method consists of following one trajectory with initial condition $y_{0}$ until a caustic is reached, saving this point $\left(t_{c}, y_{c}\right)$, and then going to a trajectory with a slightly different initial condition, $y_{0}^{\prime}$. This trajectory is also integrated until its first caustic, and the position of the second caustic $\left(t_{c}^{\prime}, y_{c}^{\prime}\right)$ is compared to the first one. In general, the caustic location will have moved by a small amount, which means that it belongs to the same fold line and does not need to be counted again (unless a new bin in the $t$ direction has been reached).

However, when the caustic locations of two initially adjacent trajectories differs by a significant amount, a different sheet of the action function has been reached, and therefore also a new fold line. It is also possible that the caustic locations differ by only a small amount, but that a cusp has been passed, and that for this reason a new fold line has been found. Therefore, $\mathbf{N}$ is also tracked. When $n_{111}$ passes through zero in quasi-2D, or when eq. (5.7) is zero in 2D (cf. sec. 5.2), a cusp has been passed. To find caustics after a caustic has already been passed (we avoid calling them second-order caustic so as not to confuse them with higher-order singularity caustics), the trajectories are simply tracked up to their second caustic, and caustics following these are treated similarly.

\subsubsection{Manifold tracking method}

A more efficient method is to track a trajectory not until a caustic but up to a fixed time $t^{\prime}$ (or a position in 2D), then tracking an adjacent trajectory up to the same time and to compare the values of $m_{11}$ (or the equivalent expression in 2D). In this way, the manifold and the value $m_{11}$ on it is obtained for the time $t^{\prime}$. The number of times that $m_{11}$ crosses zero gives the number of fold lines. This method can easily be implemented with a variable step size in the initial conditions: Whenever the values of $m_{11}$ of adjacent points on the manifold differ by more than a threshold value, a new trajectory closer to the original one is calculated, thereby refining the resolution of the manifold.

\subsubsection{Comparison of numerics and analytics for simple case 6.2}

We use as an example, $\rho(y)=\left\langle f\left(y^{\prime}\right) f\left(y^{\prime}+y\right)\right\rangle=a^{2} e^{-y^{2} / b^{2}}$, so $F_{2}=2 \sqrt{3} \frac{a}{b^{2}}$ and $F_{3}=$ $2 \sqrt{30} \frac{a}{b^{3}}$, and

$$
N_{f}(t)=\frac{\sqrt{10} \exp \left[-b^{4} /\left(24 a^{2} t^{2}\right)\right]}{b \pi}
$$

which is depicted, together with the numerics in fig. 6.2. The analytical solution accurately models the numerical values. 


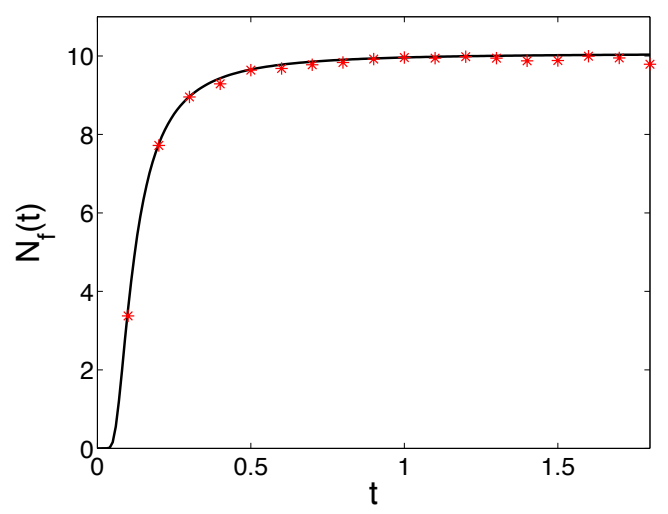

Figure 6.2: Number of fold lines for randomly distorted initial conditions $(a=0.02, b=$ 0.1 ) analytically (solid line) and numerically (red stars).

\subsection{Universal branch distribution}

We now turn to the statistics of branches in an extended random medium. As we have shown in sec. 2.4, a branch is a region of high intensity bounded by two caustics. Since experimentally one is more interested in the number of branches, we will proceed by always dividing the number of caustics by two, which is, to a very good approximation, the number of branches $N_{b}$. This is only an approximation, because of the appearance of cusp clusters: In some places, an even number of additional cusps forms close to an existing one. However, these additional cusps do not form their own extended branches, but experimentally would be identified as part of the original cusp which they belong to (see [20] for a more detailed discussion).

\subsubsection{Counting branches, first approximations}

First of all, we again shorten the notation to $m_{11} \equiv m$ and $n_{111} \equiv n$ since these are the only components of the stability matrix and tensor which will be needed. The condition for a caustic is $m=0$, which means that the mean number of branches per unit distance at time $t$ is given by

$$
N_{b}(t)=\frac{1}{2} \lim _{L \rightarrow \infty} \frac{1}{L}\left\langle\int_{0}^{L} d y \delta(m)\right\rangle
$$

or, transforming from $y$ to $y_{0}$ as in eq. (6.1), by

$$
N_{b}(t)=\frac{1}{2} \lim _{L \rightarrow \infty} \frac{1}{L}\left\langle\int_{0}^{L} d y_{0} \delta(m)\left|\partial_{y_{0}} m\right|\right\rangle=\frac{1}{2} \lim _{L \rightarrow \infty} \frac{1}{L}\left\langle\int_{0}^{L} d y_{0} \delta(m)|n|\right\rangle
$$

where the brackets denote an average over the ensemble of random potentials. Similar to eq. (6.3), it is useful to transform the $\delta$-function to $P_{c}(t)=\left\langle\delta\left(t-t_{c}\right)\right\rangle$ along the 


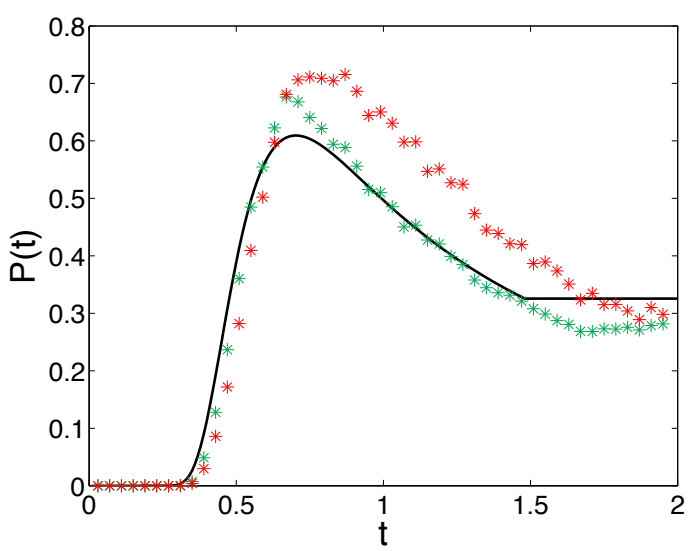

(a)

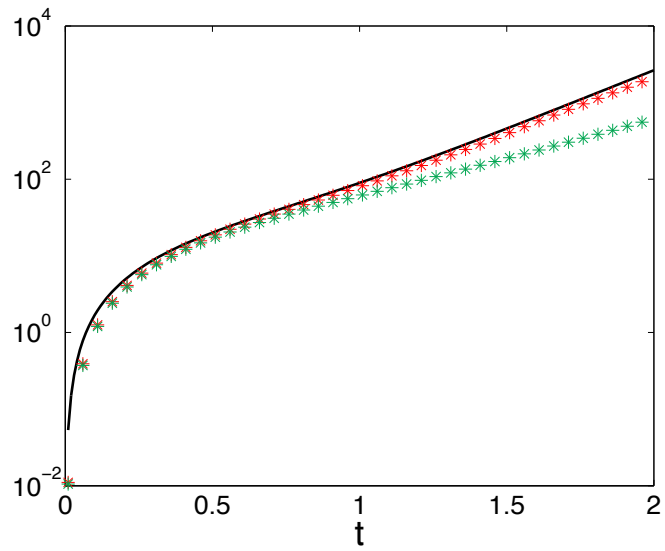

(b)

Figure 6.3: Illustration of approximations used for short-time asymptotic of $N_{c}(t)$ : (a) Comparison of $P_{c}(t)$ (analytics: black line, numerics: green dots) and $\left\langle\delta\left(t-t_{c}\right) /|\dot{m}|\right\rangle$ (red dots) multiplied by a constant for $\epsilon=0.02, \ell_{c}=0.1$ (200 realizations of the random potential). For $t$ small compared to the peak of $P_{c}(t)$, we observe that approximately $\left\langle\delta\left(t-t_{c}\right) /|\dot{m}|\right\rangle \propto P_{c}(t)$. (b) Analytical (black) and numerical (red) expression for $\sqrt{\left\langle n^{2}\right\rangle}$, together with $\langle|n|\rangle$ (green, again multiplied by a constant). This shows that for small $t$, $\langle|n|\rangle \propto \sqrt{\left\langle n^{2}\right\rangle}$ approximately.

trajectory, since we can then use the results of chapter 3 , where $P_{c}(t)$ was calculated. This is realized by

$$
\delta(m(t))=\delta\left(t-t_{c}\right) /|\dot{m}|
$$

with $t_{c}$ such that $m\left(t_{c}\right)=0$. Therefore, assuming as a first approximation that $P_{c}$ is independent of $m$ and $n$, one can write

$$
N_{f}(t)=P_{c}(t)\langle|n(t) / \dot{m}(t)|\rangle .
$$

However, it seems impossible to obtain closed equations for the second moment of $\mathrm{n} / \mathrm{m}$. For the average of the absolute value of $n / m$ this seems even more formidable, so some further approximations have to be made. We will construct short and long-time asymptotic solutions for $N_{b}(t)$, where short and long here refers to the natural time-scale of the system, which is the average time between caustics defined as (cf. eq. (3.24)

$$
t_{0}=6.27\left(2 \sigma_{1}\right)^{-1 / 3}
$$

\subsubsection{Short-term asymptotics}

For the short time limit, we assume that $\left\langle\left|\dot{m}\left(t_{c}\right)\right|\right\rangle \propto 1 / t_{0}$ at caustics and that $\langle|n(t)|\rangle$ is statistically independent and approximately proportional to $\sqrt{\left\langle n(t)^{2}\right\rangle}$. Both these 
approximations are confirmed numerically, see fig. 6.3. We can then write

$$
N_{b}(t) \approx c_{1} t_{0} P_{c}(t) \sqrt{\left\langle n^{2}(t)\right\rangle} \quad t \ll t_{0} .
$$

We note that $n(t)$ has units of inverse length because of the prefactor $\sigma_{2} / \sigma_{1}$ of eq. (5.15), which is proportional to $1 / \ell_{c}$. It also encodes the dependence on different types of correlation functions, since it varies for different functional forms of the correlation function. The universal constant $c_{1}$ is determined numerically to be $c_{1} \approx 0.033$.

\subsubsection{Long-term asymptotics}

For the long term asymptotics, we assume $m$ and $n$ to be statistically independent and their absolute value, like their even moments, to be growing exponentially. Since $P_{c} \approx$ const. for large $t$, the exponent $\lambda$ of $|n / \dot{m}|$ must be equal to the one obtained in $[83,110,111]$. We assume a similar scaling with $\sigma_{2} / \sigma_{1}$ as in the short time solution, and therefore give the long time asymptotics of the number of branches as

$$
N_{b}(t) \approx c_{2}\left(\sigma_{2} / \sigma_{1}\right) t_{0} P_{c}(t) e^{\lambda\left(t / t_{0}\right)} \quad t \gg t_{0},
$$

with $\lambda \approx 2.87$, which differs from the value used in $[83,110,111]$ because of our definition of $t_{0}$ (eq. (3.24)). This means that our result is also valid for non-Gaussian correlation functions. We numerically determine the universal constant $c_{2} \approx 0.040$.

\subsubsection{Combined solution}

Since both approximations overestimate $N_{b}$ in the regions where they are not applicable, we can construct a compound solution by always choosing the one that gives a lower value. The result is then given by

$$
\begin{aligned}
& N_{b}(t)= \begin{cases}f_{1}(t) & \text { if } f_{1}(t) \leq f_{2}(t) \\
f_{2}(t) & \text { if } f_{1}(t)>f_{2}(t)\end{cases} \\
& f_{1}(t)=c_{1} t_{0} P_{c}(t) \sqrt{\left\langle n^{2}(t)\right\rangle} \\
& f_{2}(t)=c_{2}\left(\sigma_{2} / \sigma_{1}\right) t_{0} P_{c}(t) e^{\lambda\left(t / t_{0}\right)} .
\end{aligned}
$$

From this equation, it is clear that rescaling the number of branches with $\sigma_{2} / \sigma_{1}$ and the time with $t_{0}$, the resulting curve is universal in the sense that it is independent of the parameters of the random potential and independent of the particular functional form of the correlation function.

\subsubsection{Numerical confirmation}

We perform extensive numerical simulations to confirm our theory, using a range of values for the parameters $\epsilon$ and $\ell_{c}$ and using correlation functions of Gaussian type as 


\begin{tabular}{c|c|c}
$c(|\mathbf{r}|)=c(r)$ & $\sigma_{1}^{2} \epsilon^{-2} \ell_{c}^{3}$ & $\sigma_{2}^{2} / \sigma_{1}^{2} \ell_{c}^{2}$ \\
\hline$\epsilon^{2} \exp \left(-r^{2} / \ell_{c}^{2}\right)$ & $6 \sqrt{\pi}$ & 10 \\
$\epsilon^{2} \operatorname{sech}\left(r / \ell_{c}\right)$ & 9.1876 & 10.2319 \\
$\left(1+r^{2} / \ell_{c}^{2}\right)^{-\alpha}$ & $12 \sqrt{\pi} \Gamma(\alpha+3 / 2) / \Gamma(\alpha)$ & $15+10 \alpha$
\end{tabular}

Table 6.1: Different values of $\sigma_{1}$ and $\sigma_{2} / \sigma_{1}$ for different correlation functions $c(r)$, with $\Gamma(x)$ denoting the Gamma-function [81]. Since the values for $\sigma_{1}$ and $\sigma_{2}$ of the exponentially decaying correlation function $\epsilon^{2} \operatorname{sech}\left(r / \ell_{c}\right)$ cannot be given analytically, we give the numerical approximation in this case.

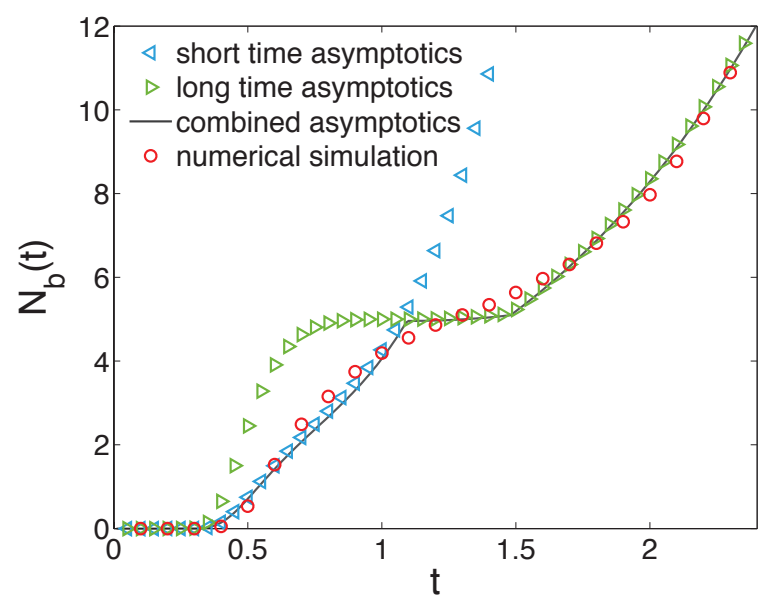

Figure 6.4: Short- and long-term asymptotics as well as compound solution of $N_{c}(t)$, numerical data for $\epsilon=4 \%$ of the particle energy and with $\ell_{c}=0.1$.

well as correlation functions with exponential and with different power law decays. We note that the correlation functions have to be six times differentiable at the origin in order for $\sigma_{2}$ to exist. The corresponding values for $\sigma_{1}$ and $\sigma_{2} / \sigma_{1}$ are given in table 6.1.

We now compare the approximations (6.5) and (6.6), the compound solution (6.7) and numerical data from one set of parameters of the random potential in fig. 6.4 for a potential with a Gaussian correlation function. We also show simulations for the different correlation functions mentioned above, and different sets of the parameters of the random potential, $\epsilon$ and $\ell_{c}$, in fig. 6.5 . We note in particular that curves with approximately the same $t_{0}$ such as the one with $\epsilon=0.08, \ell_{c}=0.1$, and the one with $\epsilon=0.04, \ell_{c}=0.06$ for the Gaussian correlation function, appear very different, although they scale with the same exponent for large $t$. In fig. 6.5p, we plot the same curves again, but rescale $t$ with $t_{0}$, and $N_{b}(t)$ with $\sigma_{2} / \sigma_{1}$, since we have predicted the resulting curve to be universal (cf. eq. (6.7)). All numerical curves collapse onto our theoretical prediction. Also included is data from a fully two-dimensional simulation (in contrast to the quasi- 


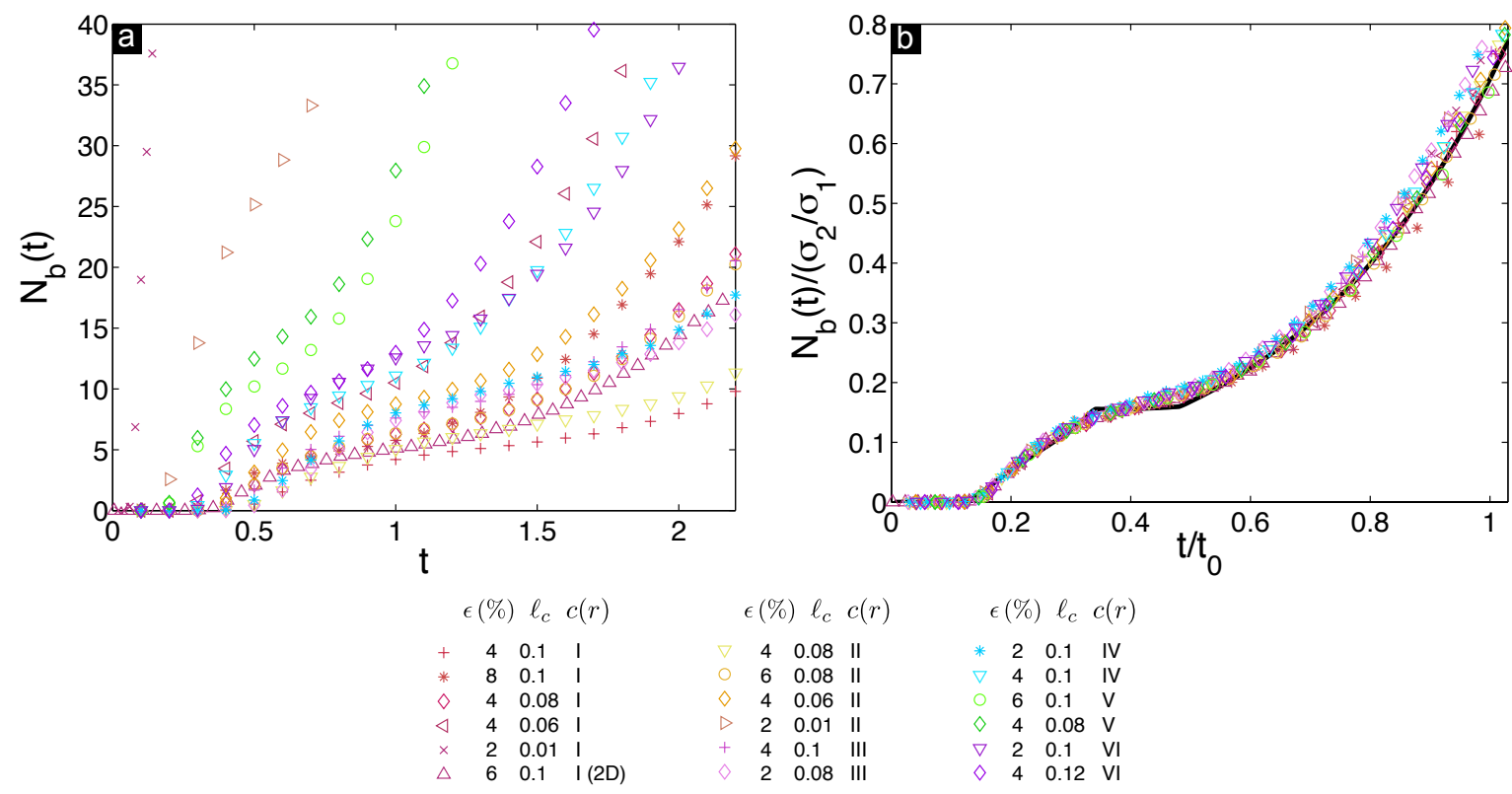

Figure 6.5: (a) $N_{b}(t)$ for different sets of parameters of the random potential ( $\epsilon$ in $\%$ of the total energy), different correlation functions, and with a fully two-dimensional simulation (2D). The type of correlation function is indicated by roman numbers: Gaussian (I), exponential (II) and power-law with exponents $\alpha=1,2,3,4$ (III, IV, V, VI) (see table 6.1 for details). (b) Same curves as in (a), but $t$ scaled by $t_{0}$ and $N_{b}(t)$ by $\sigma_{2} / \sigma_{1}$, together with analytical prediction (solid black line).

2D simulation), which fits the quasi-2D simulation and the theoretical prediction equally well. We also note the interesting shape of the universal curve. It has two inflection points, and therefore rises quickly when the first caustics become likely, but then only increases by a small amount until it reaches the purely exponential growth.

\subsection{Conclusion}

In this chapter, we have given an analytic expression for the number of branches that a flow develops in a random potential for all distances from the source by construction and combination of two asymptotic solutions, making use of the results obtained on the stability tensor in the previous chapter. We have shown that, by correct scaling, there is one universal curve for the number of branches. The time axis has to be scaled with $t_{0}$, which depends on the parameters and the functional form of the correlation function. In a similar way, the scaling in $N_{b}(t)$ depends non-trivially on integrals of the correlation function via $\sigma_{2} / \sigma_{1}$. We have demonstrated that from a measurement of the branches as a function of distance the type of correlation function cannot be inferred. However, with 
a given correlation function, not only a product of $\epsilon$ and $\ell_{c}$ (the strength and the lengthscale of the random potential) can be obtained, but they can be determined individually and uniquely. Our results therefore offer fundamental insight into the way different random potentials affect transport in random media in the regime where branched flow can be observed. 


\section{Conclusion and Outlook}

In this thesis, caustics and branched flows in random media have been studied. We have introduced methods and derived results which are applicable to a wide array of fields and beyond the examples treated here.

The results obtained can be roughly cast in two parts: In the first part (chapters 3 and 4 the emphasis was on the statistics of random caustics along trajectories under the influence of an additional magnetic field and the application of the theory to data from an experiment, while in the second part (chapters 5and 6) we developed and successfully used methods to obtain statistics on the branch density perpendicular to a flow.

In chapter 3 we reviewed existing analytical quasi-2D results obtained by deriving equations for the curvature of the action. We tested the validity of the results using fully two-dimensional simulations for a range of random potentials. We then derived equations for the probability distribution of the action curvature in the case of charged particles propagating through a random medium under the influence of a constant perpendicular magnetic field. Since this magnetic field causes focusing by itself, we were able to study the interplay between the random focusing of the disorder potential and the deterministic focusing of the magnetic field. Analytical results were obtained for the moments of the probability distribution of the mean time to a caustic as a function of the initial curvature of the Lagrangian manifold. The results were confirmed with numerical simulations. Our results are important because a real focusing device is never free from small perturbations such as a disorder potential. With our results, we can analyze to what extent focusing will be determined by the deterministic mechanism in contrast to the random one.

The theory developed in chapter 3 was used in chapter 4 to analyze data from a magnetic focusing experiment in a semiconductor microdevice. The geometry of the device in the shape of a corner adds several interesting effects compared to the more usual transverse magnetic focusing experiments. Most importantly, resistance peaks are observed because of focusing at even multiples of a reference magnetic field $B_{0}$, and because of the collimation of the beam at odd multiples of $B_{0}$. We argue that the collimation peaks are prone to being focused by a random potential even when the mean free path of the electrons is at least an order of magnitude larger than the system size. We can confirm this quantitatively using the theory of chapter 3 and additionally perform detailed simulations of the electron flow inside the device. We have been able to consistently reproduce the experimental results. Our findings have important consequences for the fabrication of ballistic semiconductor devices, since we have shown that even in very clean devices branching can strongly affect their conductance properties. 
The aim of chapters 5 and 6 was to answer the long-standing question in the theory of branched flow of how many branches appear per unit length perpendicular to the flow depending on the parameters and the type of correlation function governing the random potential. This statistics is intimately connected with the statistics of cusp points, which is the reason for deriving a detailed theory of second-order singularities of a flow in chapter 5. This has required the derivation of an extension of the stability matrix, the extended stability tensor. We subsequently derived Fokker-Planck equations for the elements of the stability matrix and the extended stability tensor in the quasi-2D approximation, and from this established and solved equations for their second moments. Not only the parameters, but also the functional form of the correlation function enters in the expression for the moments. All results were again confirmed by quasi-2D and fully $2 \mathrm{D}$ simulations.

Equipped with the results from chapters 3 and 5 , we could now study the transverse distribution of branches by breaking up the general expression for this quantity into smaller parts. This has implied using approximations for the short and long-term expressions for the branch density, but has allowed its expression in terms of quantities obtained in chapters 3 and 5 . Our results have again been confirmed with numerical simulations using random potentials with different correlation functions and parameters. The results are important for the following reasons: For the first time, a universal curve valid for a wide range of correlation functions and parameters and valid for all distances from the source has been derived. From this, one can predict quantitatively where branching occurs when the statistics of the random potential is known, or one can extract the statistics of the random potential from a measurement of the number of branches as a function of the distance from the source. Often, such as in semiconductor microdevices, it is not possible to measure the random potential directly. Our results therefore provide a novel way of obtaining the statistics of a disorder potential. Furthermore, they allow a comparison of random potentials with different types of correlation functions in terms of their effect on the branching.

As an outlook, we propose several promising continuations of the research performed in this thesis. In chapter 3, we calculated Lagrangian caustic statistics when a constant magnetic field additionally focuses trajectories. One could extend the formalism in order to treat a variable magnetic field, or a different focusing mechanism altogether, such as a lens. This would imply finding a suitable coordinate system in which a quasi-2D treatment is a good approximation of the real system. Once this is found, the calculation would proceed analogously to our derivation.

In chapter 4 we analyzed and simulated transport through a magnetic focusing device, and identified two different types of peaks, the focusing and the collimation peak. We propose to study their relationship further by construction of focusing devices with different angles of the corner. This would leave the collimation peak in place, but change the position of the caustic peaks. Also, one could study the temporal distribution of the particles transmitted, which would allow for a further experimental verification of our 
theory. We would expect the focused particles to have a broader distribution than the ones resulting from the collimation of the beam.

A natural extension of the results of chapters 5 and 6 would be to consider different initial conditions, in particular the point source, which has already been analyzed in terms of its statistics along trajectories in chapter 5 .

In addition to the extensions discussed above, we propose several further directions in which to advance the theory of branched flow. The first is to consider the shape of the fold lines. In some respects, a fold line behaves like a trajectory itself, governed implicitly by the equations of motion of the trajectories which touch the fold line. With this in mind, one could study the statistical behaviour of such a "fold trajectory", for example moments of its deviation from its initial point, and even its "velocity" distribution. Although this is a demanding problem analytically and numerically, it would yield further quantities measurable in an experiment.

We have checked the validity of the results for a wide range of correlation functions and parameters of the random potential. However, we have restricted ourselves to random potentials with a single length-scale. While this is a very good approximation in many systems, there are situations in which more realistic models are known, such as for the velocity distribution in the ocean. To our knowledge, there exists no work on branched flow in potentials with several length scales. It would be interesting to construct such a model and to analyze to what extent this can be mapped to a model with one lengthscale.

In addition to this, it would be interesting to extend our results to three dimensions in order to treat cases which cannot be approximated as two-dimensional. This could, for example, include caustics in seismic waves.

A further extension to the theory would be the inclusion of nonlinear effects in the corresponding wave equations. An example of this are Bose-Einstein-condensates, which can be described by the nonlinear Gross-Pitaevskii equation.

With this thesis, we hope to have provided a good starting-point for these further investigations and to have obtained results which will be useful to experimentalists and theoreticians in the many areas in which branched flow occurs. 



\section{A Building a catastrophe machine}

The main concepts of catastrophe theory can be illustrated by building a simple machine, for which all quantities can be calculated analytically. Several such machines have been devised in the past, of which we here use the Zeeman catastrophe machine [19,112-114]. The machine consists of a wheel (with as little friction as possible) with a pin in it, to which two elastic bands are attached. One of these is attached to a rod which can be moved freely in the plane. The other is fixed some distance away. The angle of the wheel $\theta$ is interpreted as the state variable, while the $\vec{r}=(x, y)$ location of the rod is used as the two control parameters. As in the theory of branched flow (sec. 2.4), we then have two stable catastrophes, the fold and the cusp. These are the bifurcation set of the problem, since when moving across them with the control parameters, the state variable changes non-continuously. This is the same situation as in branched flow, where moving across a caustic, one jumps to a different trajectory and thus a different state variable. The location of the catastrophes can be found as the envelopes of the contours of $\theta=$ const. which we do in fig. A.1 (the formulae can be found in [113]).

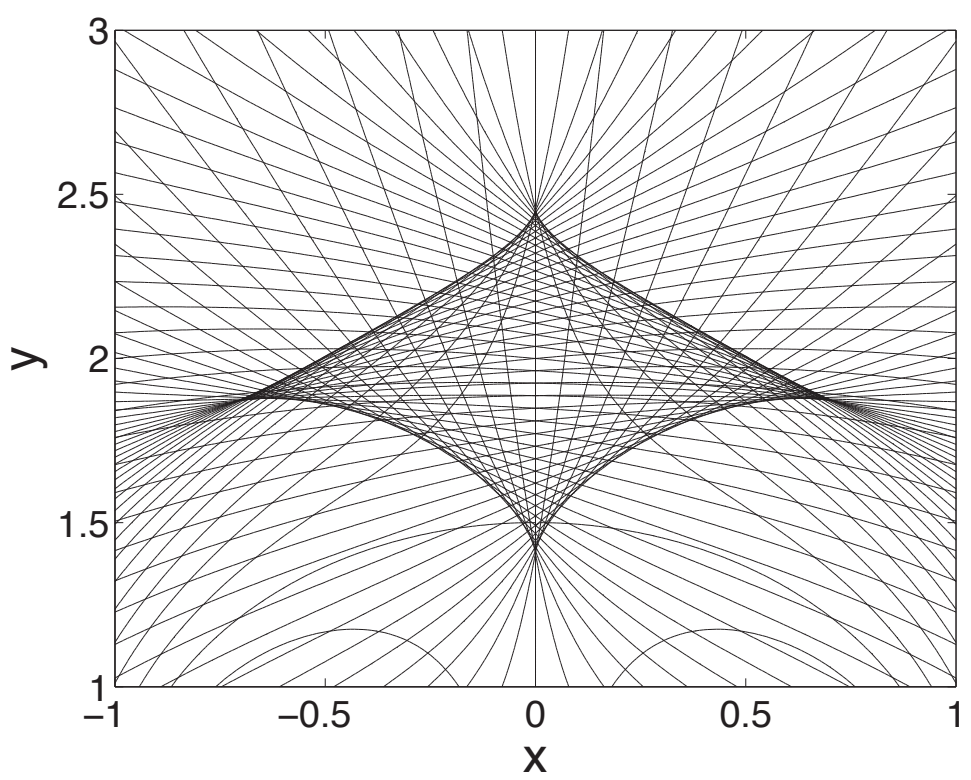

Figure A.1: Contours of $\theta=$ const. the envelopes of which constitute the catastrophe set. 


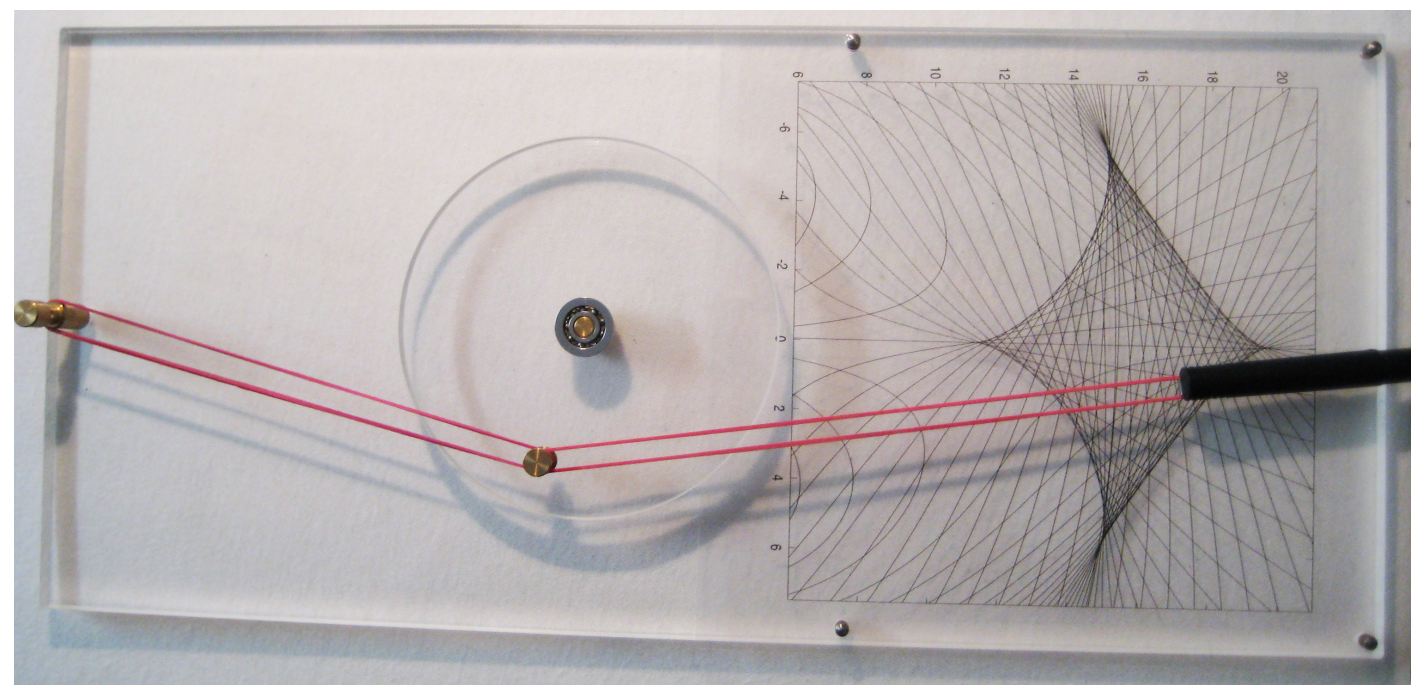

Figure A.2: Picture of the catastrophe machine.

The machine is constructed from acrylic glass with copper studs and a ball bearing to make the wheel as frictionless as possible. The catastrophe set with the correct units is photocopied onto a foil, which is attached to the machine. A picture of the experiment is shown in fig. A.2. 


\section{B The Fokker-Planck Equation}

We describe how to arrive at a Fokker-Planck equation (FPE) from a general stochastic differential equation, as given for example in $[78,115,116]$. We consider a dynamic system $\xi(t)$ (for simplicity we begin with a scalar instead of vector quantity and generalize later):

$$
\dot{\xi}(t)=h(\xi, t)+g(\xi, t) \Gamma(t)
$$

where $h(\xi, t)$ and $g(\xi, t)$ are deterministic and $\Gamma(t)$ is Gaussian random function with correlation function

$$
c\left(t, t^{\prime}\right)=\left\langle\Gamma(t) \Gamma\left(t^{\prime}\right)\right\rangle=2 \delta\left(t-t^{\prime}\right)
$$

and zero mean. We want to calculate the probability density $P(x, t)$ given the initial probability density $P\left(x_{0}, t_{0}\right)$ and eq. B.1. We know that

$$
P(x, t+\tau)=\int T\left(x, t+\tau \mid x^{\prime}, t\right) P\left(x^{\prime}, t\right) d x^{\prime}
$$

where $T\left(x, t+\tau \mid x^{\prime}, t\right)$ is the transition probability from $\left(x^{\prime}, t\right)$ to a later time and different position $(x, t+\tau)$, with $\tau>0$. The integral sums over all possibilities to get to $x$, therefore eq. (B.3) is an expression of the conservation of probability. In order to get a differential equation for $P$, we Taylor expand the integrand of eq. (B.3) in the small quantity $\Delta x=x-x^{\prime}$ by writing

$$
\begin{aligned}
T\left(x, t+\tau \mid x^{\prime}, t\right) P\left(x^{\prime}, t\right) & =T(x+\Delta x-\Delta x, t+\tau \mid x-\Delta x, t) P(x-\Delta x, t) \\
& =\sum_{0}^{\infty} \frac{(-1)^{n}}{n !}(\Delta x)^{n} \frac{\partial^{n}}{\partial x^{n}} P(x+\Delta x, t+\tau \mid x, t) P(x, t) .
\end{aligned}
$$

We insert this in eq. (B.3) and obtain

$$
P(x, t+\tau)=\int \sum_{0}^{\infty} \frac{(-1)^{n}}{n !}(\Delta x)^{n} \frac{\partial^{n}}{\partial x^{n}} P(x+\Delta x, t+\tau \mid x, t) P(x, t) d x^{\prime}
$$

which can be written in terms of the moments $M_{n}(n \geq 1)$

$$
M_{n}\left(x^{\prime}, t, \tau\right)=\left.\left\langle[\xi(t+\tau)-\xi(t)]^{n}\right\rangle\right|_{\xi(t)=x^{\prime}}=\int\left(x-x^{\prime}\right)^{n} T\left(x, t+\tau \mid x^{\prime}, t\right) d x .
$$


We obtain for

$$
\begin{aligned}
\frac{P(x, t+\tau)-P(x, t)}{\tau} & =\frac{1}{\tau} \int \sum_{1}^{\infty} \frac{(-1)^{n}}{n !}(\Delta x)^{n} \frac{\partial^{n}}{\partial x^{n}} P(x+\Delta x, t+\tau \mid x, t) P(x, t) d x^{\prime} \\
& =\frac{1}{\tau} \sum_{1}^{\infty} \frac{(-1)^{n}}{n !} \frac{\partial^{n}}{\partial x^{n}} M_{n}(x, t, \tau) P(x, t) d x^{\prime} .
\end{aligned}
$$

The moments themselves are now Taylor expanded to first order, since these are the only ones needed in the limit $\tau \rightarrow 0$ :

$$
M_{n}(x, t, \tau) / n !=D^{(n)}(x, t) \tau+\mathcal{O}\left(\tau^{2}\right),
$$

from which we now obtain

$$
\frac{\partial P(x, t)}{\partial t}=\lim _{\tau \rightarrow 0} \frac{P(x, t+\tau)-P(x, t)}{\tau}=\sum_{1}^{\infty}(-1)^{n} \frac{\partial^{n}}{\partial x^{n}} D^{(n)}(x, t) P(x, t) d x^{\prime} .
$$

For delta-correlated $\Gamma(t)$ (as in eq. (B.2)), the moments with $n>2$ vanish, and we are left with

$$
\frac{\partial P(x, t)}{\partial t}=\left[-\frac{\partial}{\partial x} D^{(1)}(x, t)+\frac{\partial^{2}}{\partial x^{2}} D^{(2)}(x, t)\right] P(x, t)
$$

which is the Fokker-Planck equation. We also give the multi-dimensional case, for the transition probability $P\left(x, t \mid x_{0}, t_{0}\right)$, which equally obeys the FPE with initial condition

$$
P\left(x, t_{0} \mid x_{0}, t_{0}\right)=\delta\left(x-x_{0}\right) .
$$

We assume a vector equation with components

$$
\dot{\xi}_{i}(t)=h_{i}(\vec{\xi}, t)+g_{i j}(\vec{\xi}, t) \vec{\Gamma}_{j}(t)
$$

with the multidimensional noise

$$
\left\langle\Gamma_{i}(t) \Gamma_{j}\left(t^{\prime}\right)\right\rangle=2 \delta_{i j} \delta\left(t-t^{\prime}\right)
$$

and zero mean. It is given by (summation convention implied)

$$
\frac{\partial P\left(\vec{x}, t \mid \vec{x}_{0}, t_{0}\right)}{\partial t}=\left[-\frac{\partial}{\partial x_{i}} D_{i}^{(1)}(\vec{x}, t)+\frac{\partial^{2}}{\partial x_{i} \partial x_{j}} D_{i j}^{(2)}(\vec{x}, t)\right] P\left(\vec{x}, t \mid \vec{x}_{0}, t_{0}\right)
$$

with the drift and diffusion coefficients given by

$$
\begin{aligned}
& D_{i}^{(1)}(\vec{x}, t)=h_{i}(\vec{x}, t)+g_{k j}(\vec{x}, t) \partial x_{k} g_{i j}(\vec{x}, t) \\
& D_{i j}^{(2)}(\vec{x}, t)=g_{i k}(\vec{x}, t) g_{j k}(\vec{x}, t) .
\end{aligned}
$$


We note that the FPE can also be written as a conservation equation for a probability current. For the one-dimensional case we rewrite eq. (B.4) as

$$
\frac{\partial P}{\partial t}+\frac{\partial J}{\partial x}=0
$$

with the probability current $J$.

$$
J=\left[D^{(1)}(x, t)-\frac{\partial}{\partial x} D^{(2)}(x, t)\right] P(x, t)
$$

Finally, we note that there is an equivalent backward equation to the forward FPE given here. In the forward FPE, initial conditions for the probability density at time $t_{0}$ are given, and the FPE describes the time evolution for this density for $t \geq t_{0}$. In some cases, most notably mean first passage time calculations, one wants to fix the final conditions and leave the initial conditions unspecified. One can then write down a FPE for the reverse time evolution valid for $t_{0} \leq t$ (where $t$ is the final time). The backward FPE for the transitional probability $P\left(\vec{x}, t \mid \vec{x}_{0}, t_{0}\right)$ is given by

$$
\frac{\partial P\left(\vec{x}, t \mid \vec{x}_{0}, t_{0}\right)}{\partial t_{0}}=\left[-D_{i}^{(1)}\left(\vec{x}_{0}, t_{0}\right) \frac{\partial}{\partial x_{i}^{0}}+D_{i j}^{(2)}\left(\vec{x}_{0}, t_{0}\right) \frac{\partial^{2}}{\partial x_{i}^{0} \partial x_{j}^{0}}\right] P\left(\vec{x}, t \mid \vec{x}_{0}, t_{0}\right)
$$

with final condition

$$
P\left(x, t \mid x_{0}, t\right)=\delta\left(x-x_{0}\right) .
$$




\section{Distribution incident on QPC}

We assume that there is a lead attached to the emitting QPC which guides the electrons to the QPC. What is a reasonable assumption for the distribution of the number of particles (or the intensity) for some angle $\alpha$, i.e. $I(\alpha)$, inside lead? This is needed as initial condition when simulating a complex geometry such as the QPC.

Since we want to simulate a bath at the end of the lead, we assume that all directions are equally present. To calculate the resulting angular distribution in the lead, we look at a small, straight area in the lead as illustrated in fig C.1.

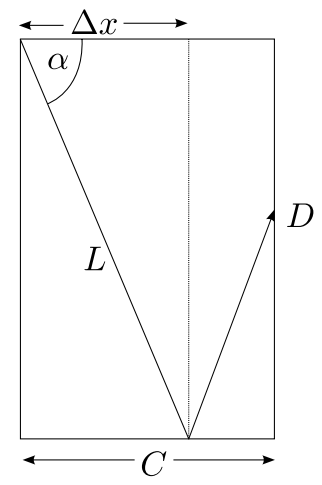

Figure C.1: trajectory in a small area in a straight lead

Since all particles have the same energy, they also have the same velocity. The contribution of one electron to the current density inside the small area $C \times D$ is therefore proportional to the length of the trajectory inside the area. This total length is given by $L \frac{C}{\Delta x}$. So the contribution to the density of one single particle to the current density in this area is given by

$$
\Delta \rho=L \frac{C}{\Delta x}=\frac{L C}{L \cos (\alpha)}=\frac{C}{\cos (\alpha)}
$$

For a particle at angle $\alpha$ to contribute equally to the current density, one has to multiply its contribution to the density by $\cos (\alpha)$ such that $\Delta \rho=$ const. When simulating trajectories emerging from a lead, this means that one has to start trajectories with a cosine distribution in order to simulate a constant flux through the lead. 


\section{Model potential at walls and QPCs of corner device}

We construct a simple model of the soft walls and a model of the QPC which describes the potential of the QPC as a function of $x$ and $y$. For the walls we use a quadratic equation with parameter $\omega$

$$
V_{s w}(x, y)= \begin{cases}g(x) & \text { if } x<x_{0} \\ 0 & \text { if } x \geqslant x_{0}\end{cases}
$$

with

$$
g\left(x, x_{0}\right)=\frac{1}{2} w^{2}\left(x-x_{0}\right)^{2} .
$$

The QPC without a saddle point potential is modeled by the function (see below for the additional saddle point equations)

$$
V_{Q P C}(x, y)= \begin{cases}f(x, y, \alpha, h) & \text { if } y<x_{0} \\ 0 & \text { if } y \geqslant x_{0}\end{cases}
$$

with

$$
f(x, y, \alpha, h)=\frac{1}{2}\left\{\tanh \left(\alpha\left(x-1-\frac{h(y)}{2}\right)\right)+\tanh \left(\alpha\left(1-\frac{h(y)}{2}-x\right)\right)\right\}+1
$$

where the parameter $\alpha$ describes the steepness of the potential at the edges of the QPC. The function $h(y)$ describes the opening of the potential of the QPC into the system. We choose it to be quadratic here, such that $h(y)=h_{0}+\beta y^{2}$. To get one side of the potential, we multiply $V_{s w}(x, y) \times V_{Q P C}(y, x)$ in order to get

$$
V_{\text {combined }_{x}}(x, y)= \begin{cases}g\left(x, x_{0}\right) f(x, y, \alpha, h) & \text { if } x<x_{0} \\ 0 & \text { if } x \geqslant x_{0}\end{cases}
$$

The complete potential is then a superposition of the combined $x$ and $y$ potentials.

$$
V(x, y)= \begin{cases}g\left(x, x_{0}\right) f(y, x, \alpha, h)+g\left(y, x_{0}\right) f(x, y, \alpha, h) & \text { if } x<x_{0} \wedge y<x_{0} \\ g\left(y, x_{0}\right) f(x, y, \alpha, h) & \text { if } x \geqslant x_{0} \wedge y<x_{0} \\ g\left(x, x_{0}\right) f(y, x, \alpha, h) & \text { if } x<x_{0} \wedge y \geqslant x_{0} \\ 0 & \text { if } y \geqslant x_{0} \wedge y \geqslant x_{0}\end{cases}
$$


The force is given by $\mathbf{F}=-\nabla V$. We give the force in the $x$-direction as an example:

$$
F_{x}= \begin{cases}\frac{1}{2} g\left(x, x_{0}\right)\left(u^{-}\left(x, y, \alpha, \beta, h_{0}\right) \beta x+u^{+}\left(x, y, \alpha, \beta, h_{0}\right) \beta x\right) & \\ +\frac{1}{2} g\left(y, x_{0}\right)\left(u^{-}\left(y, x, \alpha, \beta, h_{0}\right)+u^{+}\left(y, x, \alpha, \beta, h_{0}\right)\right) & \\ +w^{2}\left(x-x_{0}\right)\left(1+\frac{1}{2}\left(v^{-}\left(x, y, \alpha, \beta, h_{0}\right)+v^{+}\left(x, y, \alpha, \beta, h_{0}\right)\right)\right) & \frac{\sqrt{2}}{w}-x>0 \wedge \frac{\sqrt{2}}{w}-y>0 \\ \frac{1}{2} g\left(y, x_{0}\right)\left(u^{-}\left(y, x, \alpha, \beta . h_{0}\right)-u^{+}\left(y, x, \alpha, \beta . h_{0}\right)\right) & \frac{\sqrt{2}}{w}-x \leq 0 \wedge \frac{\sqrt{2}}{w}-y \geq 0 \\ \frac{1}{4} w^{2}\left(x-\frac{\sqrt{2}}{w}\right)^{2}\left(u^{-}\left(x, y, \alpha, \beta, h_{0}\right) \beta x+u^{+}\left(x, y, \alpha, \beta, h_{0}\right) \beta x\right) & \\ +w^{2}\left(x-\frac{\sqrt{2}}{w}\right)\left(1+\frac{1}{2}\left(v^{-}\left(x, y, \alpha, \beta, h_{0}\right)+v^{+}\left(x, y, \alpha, \beta, h_{0}\right)\right)\right) & \frac{\sqrt{2}}{w}-x \geq 0 \wedge \frac{\sqrt{2}}{w}-y \leq 0\end{cases}
$$

with

$$
\begin{aligned}
& u^{-}\left(x, y, \alpha, \beta, h_{0}\right)=-\alpha \operatorname{sech}^{2}\left(\alpha\left(\frac{1}{2}\left(-\beta x^{2}-h_{0}\right)-y+1\right)\right) \\
& u^{+}\left(x, y, \alpha, \beta, h_{0}\right)=-\alpha \operatorname{sech}^{2}\left(\alpha\left(\frac{1}{2}\left(-\beta x^{2}-h_{0}\right)+y-1\right)\right) \\
& v^{-}\left(x, y, \alpha, \beta, h_{0}\right)=\tanh \left(\alpha\left(\frac{1}{2}\left(-\beta x^{2}-h_{0}\right)-y+1\right)\right) \\
& v^{+}\left(x, y, \alpha, \beta, h_{0}\right)=\tanh \left(\alpha\left(\frac{1}{2}\left(-\beta x^{2}-h_{0}\right)+y-1\right)\right)
\end{aligned}
$$

In order to model the saddle points inside the QPC, instead of the prefactor of $1 / 2$ in front of the hyperbolic tangent in eq. (D.2), we now use $\frac{1}{2}-g_{0} e^{-\left(x-x_{0}\right)^{2} / s}$ with parameters $g_{0}$ and $s$. This will be $1 / 2$ for most of the time except for the region between the QPCs. The walls of the chamfered corner are modeled in the exactly the same way as the other walls.

The numerical values of the parameters used for the final results of the simulations are $\alpha=\omega=80, \beta=-2.7, s=0.001$. The values the initial opening of the $\mathrm{QPC}, h_{0}=.22$ is known from the experiment. The saddle point parameters $g_{0}=0.8$ is used, except for the section comparing the different saddle strengths. 


\section{Bibliography}

[1] Thomas (transl.), I. Greek Mathematical Works: Volume II, From Aristarchus to Pappus. (Harvard University Press, 1993).

[2] Peregrine, D. H. Interaction of water waves and currents. Adv. Appl. Mech. 16, 9-117 (1976).

[3] Gerber, M. The interaction of deep-water gravity waves and an annular current: linear theory. J. Fluid Mech. 248, 153-172 (1993).

[4] Gerber, M. Giant waves and the agulhas current. Deep Sea Res. (1996).

[5] Lavrenov, I. V. The wave energy concentration at the agulhas current off south africa. Natural Hazards 17, 117-127 (1998).

[6] Berry, M. Tsunami asymptotics. New J. Phys. 7, 129 (2005).

[7] Berry, M. Focused tsunami waves. Proc. R. Soc. A 463, 3055-3071 (2007).

[8] Zahibo, N. et al. The 1867 virgin island tsunami. Natural Hazards and Earth System Sciences 3, 367-376 (2003).

[9] Ioualalen, M., Pelinovsky, E., Asavanant, J., Lipikorn, R. \& Deschamps, A. On the weak impact of the 26 december indian ocean tsunami on the bangladesh coast. Natural Hazards and Earth System Sciences 7, 141-147 (2007).

[10] Pierce, A. D. \& Maglieri, D. J. Effects of atmospheric irregularities on sonic-boom propagation. J. Acoust. Soc. Am. 51, 702-721 (1972).

[11] Čerenkov, P. A. Visible radiation produced by electrons moving in a medium with velocities exceeding that of light. Phys. Rev. 52, 378-379 (1937).

[12] Kleinknecht, K. Detectors for Particle Radiation (Cambridge University Press, 1998).

[13] Descartes, R. Discours de la methode (1637).

[14] Airy, G. On the intensity of light in the neighbourhood of a caustics. Trans. Cambridge Philos. Soc. 6, 379-402 (1838). 
[15] Arnold, V. I. Singularities of smooth mappings. Russian Math. Surveys 1-43 (1969).

[16] Arnold, V. I. Mathematical Methods of Classical Mechanics (Springer, 1978).

[17] Thom, R. Structural stability and morphogenesis (Addison-Wesley, 1989).

[18] Zeeman, E. C. Catastrophe theory, selected papers (Addison-Wesley Publishing Co., Reading, Massachusetts, 1977).

[19] Poston, T. \& Stewart, I. Catastrophe Theory and its Applications (Dover, 1978).

[20] Berry, M. \& Upstill, C. Catastrophe optics: Morphologies of caustics and their diffraction patterns. Progress in Optics 18, 257-346 (1980).

[21] Golubitsky. An introduction to catastrophe theory and its applications. SIAM Review 20, 352-387 (1978).

[22] Stewart, I. Catastrophe theory in physics. Rep. Prog. Phys. 45, 185-221 (1982).

[23] Jakeman, E., Pike, E. R. \& Pusey, P. N. Photon correlation study of stellar scintillation. Nature 263, 215-217 (1976).

[24] Berry, M. V. Focusing and twinkling: critical exponents from catastrophes in non gaussian random short waves. J. Phys. A 10, 2061-2081 (1977).

[25] Kravtsov, Y. \& Orlov, Y. Geometrical Optics of Inhomogeneous Media (Springer, 1990).

[26] Kravtsov, Y. A. Propagation of electromagnetic waves through a turbulent atmosphere. Reports on Progress in Physics 55, 39-113 (1992).

[27] Salomons, E. M. Caustic diffraction fields in a downward refracting atmosphere. The Journal of the Acoustical Society of America 104, 3259-3272 (1998).

[28] Solli, D. R., Ropers, C., Koonath, P. \& Jalali, B. Optical rogue waves. Nature 450, 1054-1057 (2007).

[29] Longuet-Higgins, M. S. \& Stewart, R. W. The changes in amplitude of short gravity waves. J. Fluid Mech. 10, 529-549 (1961).

[30] Lindgren, L. Some properties of a normal process near a local maximum. Ann. Math. Statist. 41, 1870-1883 (1970).

[31] Phillis, O. M., Gu, D. \& Donelan, M. Expected structure of extreme waves in a gaussian sea. part i : Theory and swade buoy measurements. J. Phys. Oceanogr. 23, 992-1000 (1993). 
[32] Phillis, O. M., Gu, D. \& Donelan, M. On the expected structure of extreme waves in a gaussian sea. part ii : Swade scanning radar altimeter measurements. J. Phys. Oceanogr. 23, 2297-2309 (1993).

[33] Kharif, C. \& Pelinovsky, E. Physical mechanisms of the rogue wave phenomenon. European Journal of Mechanics - B/Fluids 22, 603 - 634 (2003).

[34] Onorato, M., Osborne, A. R., Serio, M. \& Bertone, S. Freak waves in random oceanic sea states. Phys. Rev. Lett. 86, 5831-5834 (2001).

[35] Brown, M. G. Space-time surface gravity wave caustics: structurally stable extreme wave events. Wave Motion 33, 117 - 143 (2001).

[36] Müller, P., Garrett, C. \& Osborne, A. Rogue waves. Oceanography 18, 66-75 (2005).

[37] Heller, E. J., Kaplan, L. \& Dahlen, A. Refraction of a gaussian seaway. J. Geophys. Res. 113, C09023 (2008).

[38] Ostashev, V. E. Sound propagation and scattering in media with random inhomogeneities of sound speed, density and medium velocity. Waves in Random Media 4, 403-428 (1994).

[39] Blanc-Benon, P., Juvé, D., Ostashev, V. E. \& Wandelt, R. On the appearance of caustics for plane sound-wave propagation in moving random media. Waves in Random Media 5, 183-199 (1995).

[40] Vadov, R. Observation and location of caustics in the ocean at different sound frequencies. Acoustical Physics 43, 401-405 (1997).

[41] Wolfson, M. \& Tappert, F. Study of horizontal multipaths and ray chaos due to ocean mesoscale structure. J. Acoust. Soc. Am. 107, 154-162 (2000).

[42] Wolfson, M. \& Tomsovic, S. On the stability of long-range sound propagation through a structured ocean. J. Acous. Soc. Am. 109, 2693 (2001).

[43] Brown, M. G. et al. Ray dynamics in long-range deep ocean sound propagation. The Journal of the Acoustical Society of America 113, 2533-2547 (2003).

[44] Gribova, E. Z. Statistics of caustics in an underwater sound channel. Acoustical Physics 53, 730-735 (2007).

[45] Topinka, M. A. et al. Coherent branched flow in a two-dimensional electron gas. Nature 410, 183-186 (2001).

[46] Jura, M. P. et al. Unexpected features of branched flow through high-mobility two-dimensional electron gases. Nature Physics 3, 841 - 845 (2007). 
[47] Aidala, K. E. et al. Imaging magnetic focusing of coherent electron waves. Nature Physics 3, 464 (2007).

[48] Wilkinson, M., Mehlig, B. \& Bezuglyy, V. Caustic activation of rain showers. Phys. Rev. Lett. 97, 048501 (2006).

[49] Höhmann, R., Kuhl, U., Stöckmann, H.-J., Kaplan, L. \& Heller, E. J. Freak waves in the linear regime: A microwave study. Phys. Rev. Lett. 104, 093901 (2010).

[50] Goldstein, H. Klassische Mechanik (Akademische Verlagsgesellschaft, Frankfurt am Main, 1963).

[51] Gelfand, I. \& Fomin, S. Calculus of Variations (Dover, 1963).

[52] Landau, L. D. \& Lifschitz, E. M. Lehrbuch der theoretischen Physik, vol. 2 (Verlag Harri Deutsch, 1997).

[53] Courant, R. \& Hilbert, D. Methods of Mathematical Physics, vol. 2 (WileyInterscience, 1962).

[54] Whitham, G. B. Linear and Nonlinear Waves (Wiley \& Sons, Inc., 1974).

[55] Delgado, M. The lagrange-charpit method. SIAM Review 39, 298-304 (1997).

[56] Littlejohn, R. G. The van-vleck formula, maslov theory, and phase space geometry. J. Stat. Phys. 68, 7-50 (1992).

[57] Arnold, V. I. Lagrangian manifolds with singularities, asymptotic rays, and the open swallowtail. Functional Analysis and Its Applications 15, 235-246 (1981).

[58] Guggenheimer, H. Differential Geometry (Dover, 1977).

[59] Berry, M. V. \& Hannay, J. H. Umbilic points on a gaussian random surface. J. Phys. A 10, 2083-2091 (1977).

[60] Adler, R. J. \& Taylor, J. E. Random Fields and Geometry (Springer, 2007).

[61] Christakos, G. Random Field Models in Earth Sciences (Dover, 1992).

[62] Bröking, K. Transport in Micro-Hallbars. Master's thesis, Georg-AugustUniversität Göttingen (2006).

[63] Arnold, V. I. Catastrophe theory (Springer, 2004).

[64] Heller, E. J. Wave packet dynamics and quantum chaology. In Giannoni, M.-J., Voros, A. \& Zinn-Justin, J. (eds.) Chaos and quantum physics, 549-663 (Elsevier Science Publishers, 1991). 
[65] Eckhardt, B. \& Wintgen, D. Indices in classical mechanics. J. Phys. A 24, 43354348 (1991).

[66] Benamou, J.-D. An introduction to eulerian geometrical optics (1992-2002). J. Sci. Comput. 19, 63-93 (2003).

[67] Beck, C. \& Schlögl, F. Thermodynamics of chaotic systems: an introduction (Cambridge University Press, 1997).

[68] Cvitanović, P., Artuso, R., Mainieri, R., Tanner, G. \& Vattay, G. Chaos: Classical and Quantum (Niels Bohr Institute, 2005).

[69] Malakhov, A. N. \& Saichev, A. I. Lagrangian and eulerian descriptions of the statistical properties of light waves. Radiofizika 19, 1368-1377 (1976).

[70] Granger, R. Fluid Mechanics (Dover, 1995).

[71] White, B. S. The stochastic caustic. SIAM Journal on Applied Mathematics 44, 127-149 (1984).

[72] White, B. S. \& Fornberg, B. On the chance of freak waves at sea. J. Fluid Mech. 355, 113-138 (1998).

[73] Klyatskin, V. I. Caustics in random media. Waves in Random Media 3, 93-100 (1993).

[74] Klyatskin, V. I. Stochastic equations through the eye of the physicist (Elsevier, 2005).

[75] Kulkarny, V. \& White, B. Focusing of waves in turbulent inhomogeneous media. Phys. Fluids 25, 1770-1784 (1982).

[76] Siegert, A. J. F. On the first passage time probability problem. Physical Review 81, 617 (1951).

[77] Stratonovich. Topics in the Theory of Random Noise, vol. 1 (Gordon and Breach, 1963).

[78] Gardiner, C. Handbook of Stochastic Methods (Springer, 2004).

[79] Feller, W. The parabolic differential equations and the associated semi-groups of transformations. Ann. Math. 55, 468 (1952).

[80] Polyanin, A. \& Zaitsev, V. Handbook of Exact Solutions for Ordinary Differential Equations (CRC Press, 2002). 
[81] Abramowitz, M. \& Stegun, I. A. Handbook of Mathematical Functions (National Bureau of Standards, 1972).

[82] Nair, B. \& White, B. S. High-frequency wave propagation in random media-a unified approach. SIAM Journal on Applied Mathematics 51, 374-411 (1991).

[83] Kaplan, L. Statistics of branched flow in a weak correlated random potential. Phys. Rev. Lett. 89, 184103 (2002).

[84] Sharvin, Y. V. \& Fisher, L. M. Observation of focused electron beams in a metal. JETP Lett. 1, 152-153 (1965).

[85] Tsoi, V. Focusing of electrons in a metal by a transverse magnetic field. JETP Lett. 19, 70-71 (1974).

[86] van Son, P. C., van Kempen, H. \& Wyder, P. New method to study the electronphonon interaction in metals. Phys. Rev. Lett. 58, 1567-1570 (1987).

[87] van Houten et al., H. Coherent electron focusing with quantum point contacts in a two-dimensional electron gas. Phys. Rev. B 39, 8556-8576 (1989).

[88] Beenakker, C. W. J. \& van Houten, H. Billiard model of a ballistic multiprobe conductor. Phys. Rev. Lett. 63, 1857-1860 (1989).

[89] van Houten, H., Beenakker, C. W. J. \& van Wees, B. J. Semiconductors and Semimetals (Academic, 1992).

[90] Rokhinson, L. P., Larkina, V., Lyanda-Geller, Y. B., Pfeiffer, L. N. \& West, K. W. Spin separation in cyclotron motion. Phys. Rev. Lett. 93, 146601 (2004).

[91] Landauer, R. Spatial variation of currents and fields due to localized scatterers in metalic conduction. IBM J. Res. Dev. 1, 233 (1957).

[92] Landauer, R. Electrical resistance of disordered one-dimensional lattices. Philos. Mag. 21, 863 (1970).

[93] Büttiker, M. Absence of backscattering in the quantum hall effect in multiprobe conductors. Phys. Rev. B 38, 9375-9389 (1988).

[94] Metzger, J. J. et al. Ballistic transport in corner device. in preparation (2010).

[95] Molenkamp, L. W. et al. Electron-beam collimation with a quantum point contact. Phys. Rev. B 41, 1274-1277 (1990).

[96] Davies, J. H. The Physics of Low-dimensional Semiconductors (Cambridge University Press, 2006). 
[97] Datta, S. Electronic Transport in Mesoscopic Systems (Cambridge University Press, 2003).

[98] Gutzwiller, M. C. Chaos in Classical and Quantum Mechanics (Springer, 1990).

[99] Heller, E. \& Vanicek, J. A new class of semiclassical wave function uniformizations. Phys. Rev. E 64 (2001).

[100] Jetschke, G. Mathematik der Selbstorganisation (Harri Deutsch, 1989).

[101] Shaw, S. E. J. Propagation in Smooth Random Potentials. Ph.D. thesis, Havard University, Cambridge, Massachusetts (2002).

[102] Papanicolaou, G. C. \& Kohler, W. Asymptotic theory of mixing stochastic ordinary differential equations. Comm. Pure Appl. Math. 27, 641-668 (1974).

[103] Dawson, D. A. \& Papanicolau, G. C. A random wave process. Appl. Math. Optim. 12, 97-114 (1984).

[104] Hesselink, L. \& Sturtevant, B. Propagation of weak shocks through a random medium. Journal of Fluid Mechanics 196, 513-553 (1988).

[105] Oksendal, B. Stochastic differential equations (Springer, 1998).

[106] Metzger, J. J., Fleischmann, R. \& Geisel, T. Universal statistics of branched flows. Phys. Rev. Lett. 105, 020601 (2010).

[107] Benamou, J.-D. \& Solliec, I. An eulerian method for capturing caustics. Journal of Computational Physics 162, 132 - 163 (2000).

[108] Benamou, J. D., Lafitte, O., Sentis, R. \& Solliec, I. A geometrical optics-based numerical method for high frequency electromagnetic fields computations near fold caustics-part i. Journal of Computational and Applied Mathematics 156, 93 - 125 (2003).

[109] Benamou, J. D., Lafitte, O., Solliec, I. \& Sentis, R. A geometric optics method for high-frequency electromagnetic fields computations near fold caustics-part ii. the energy. Journal of Computational and Applied Mathematics 167, 91 - 134 (2004).

[110] Aleiner, I. L. \& Larkin, A. I. Divergence of classical trajectories and weak localization. Phys. Rev. B 54, 14423-14444 (1996).

[111] Schomerus, H. \& Titov, M. Statistics of finite-time lyapunov exponents in a random time-dependent potential. Phys. Rev. E 66, 066207 (2002).

[112] Zeeman, E. C. A catastrophe machine. In Waddington, C. H. (ed.) Towards a Theoretical Biology (Edinburgh University Press, 1968-72). 
[113] Poston, T. \& Woodcock, A. Zeeman's catastrophe machine. Proc. Camb. Phil. Soc. 74, 217 (1973).

[114] Dubois, J.-G. \& Dufour, J.-P. La théorie des catastrophes i. la machine à catastrophes. Ann. Inst. Henri Poincaré 20, 135-151 (1974).

[115] Risken, H. The Fokker-Planck Equation (Springer, 1996).

[116] van Kampen, N. G. Stochastic processes in physics and chemistry (Elsevier, 2007). 


\section{Acknowledgements}

It is my sincere pleasure to thank Theo Geisel for the opportunity to work in his group, for his support and advice not only in scientific matters, but also in the musical and even medical domain. His indefatigable efforts for the thriving of the group have created a unique atmosphere which permeates every aspect of life at the MPI for Dynamics and Self-Organization.

I wish to thank Ragnar Fleischmann for his guidance and support, for his persistent optimism, insight and enthusiasm, and for the many inspiring discussions. I have learnt more over the past years than I could have imagined.

I thank the other members of the mesoscopic systems group, Oliver Bendix, Kai Bröking and Holger Hennig, for enlightening discussions. In particular, I would like to thank Kai for providing me with his interpolation code, and Oliver for the hours of fun in the geometry club.

I acknowledge the collaboration with Denis Maryenko and Jurgen Smet from the MPI for Solid State Research in Stuttgart, and thank them for the data they provided and for a very interesting visit of their institute.

I acknowledge support from the DFG Forschergruppe 760, and thank their members for the many interesting conferences and seminars.

I thank the other two inhabitants of the think tank, Wolfgang Keil and Christoph Kirst, for the unique atmosphere, the countless discussions and the musical activities. I would like to thank Wolfgang for teaching me the importance of the beat. I also wish to thank Peter Hiemeyer, Michael Monteforte and Olav Stetter for the many extracurricular activities, and Stefan Eule for a great time at the APS conferences.

I thank the computer and support crew, Denny Fliegner, Yorck-Fabian Beensen and Barbara Guichemer, the first two for help with hard- and software, the latter two for providing me with different devices for the input-impaired. I also wish to thank Corinna Trautsch, Tanja Gindele, Katharina Jeremias and Regina Wunderlich for their support with administrative matters, and everyone else from the group for the friendly atmosphere.

I would like to thank Johanna for her loving patience, my sisters, and of course my parents for their unfailing support of my plans, past and future. 



\section{Lebenslauf}

Jakob Johannes Metzger, Master in Science geboren am 25. 12. 1982 in Bonn

Staatsangehörigkeit: deutsch

1989-2001 Schulbesuche in Bonn und Brüssel

07/2001 Europäisches Baccalaureat, Europäische Schule Brüssel

08/2001-08/2003 Studium der Physik, Imperial College, London

09/2003-07/2004 Studium der Physik, Universität Freiburg

08/2004-07/2005 Studium der Physik, Imperial College, London

07/2005 Master in Science, Imperial College

Titel der Masterarbeit: "Efficiency studies of the COMPASS Spectrometer" Betreuer: Prof. Dr. K. Königsmann, Freiburg

seit 11/2005 Promotion am Max-Planck-Institut für Dynamik

und Selbstorganisation, Göttingen und

Institut für nichtlineare Dynamik, Universität Göttingen 